\title{
Synthesis of $\pi$-Extended Imidazoles by 1,3-Dipolar Cycloaddition of Polycyclic Aromatic Azomethine Ylides with Nitriles
}

\author{
Qiang-Qiang Li, Kotaro Ochiai, Cherie-Anne Lee, Shingo Ito* \\ Division of Chemistry and Biological Chemistry, School of Physical and Mathematical Sciences, \\ Nanyang Technological University, 21 Nanyang Link, Singapore 637371, Singapore
}

Table of Contents

1. Experimental Section $\quad$ S2

2. NMR Spectra $\quad \mathrm{S} 14$

3. X-Ray Crystallographic Data S35

$\begin{array}{ll}\text { 4. Optical Properties } & \text { S37 }\end{array}$

5. Electrochemical Calculations $\quad$ S39

6. Theoretical Calculations $\quad$ S40 


\section{Experimental Section}

General: Reactions were carried out under nitrogen atmosphere using standard Schlenk techniques. Thin-layer chromatography (TLC) was performed using glass plates pre-coated with silica gel impregnated with a fluorescent indicator (Merck, \#1.15685.0001). Silica gel column chromatography was performed as described by Still, et al., ${ }^{1}$ employing (Davisil, $60 \AA$, 40-63 micron) purchased from Sigma-Aldrich.

Instrumentation: NMR spectra were recorded on Bruker AV $500\left({ }^{1} \mathrm{H}: 500 \mathrm{MHz}\right.$ and $\left.{ }^{13} \mathrm{C}: 126 \mathrm{MHz}\right)$, AV $400\left({ }^{1} \mathrm{H}\right.$ : $400 \mathrm{MHz}$ and $\left.{ }^{13} \mathrm{C}: 100 \mathrm{MHz}\right)$ and BBFO $\left({ }^{1} \mathrm{H}: 400 \mathrm{MHz}\right.$ and $\left.{ }^{13} \mathrm{C}: 100 \mathrm{MHz}\right) \mathrm{NMR}$ spectrometers. Chemical shift values for protons are referenced to the signal of tetramethylsilane $(\delta$ $0.00)$, the residual signal of chloroform- $d(\delta$ 7.26) chemical shift values for carbons are referenced to the signal of tetramethylsilane $(\delta 0.00)$ or the carbon resonance of chloroform- $d(\delta 77.2)$. Highresolution mass (HRMS) spectra were taken on a Waters Q-Tof Premier mass spectrometer with the electron spray ionization time-of-flight (ESI-TOF) method. Infrared (IR) spectra were recorded on a Shimadzu FTIR-8400 spectrometer with an attenuated total reflection (ATR) system. Ultraviolet/visible (UV/vis) absorption spectra were recorded on a Shimadzu UV-3100 spectrometer. Fluorescence spectra were recorded on JASCO FP-8500 Spectrofluorometer. Quantum yields were determined by relative method with Fluorescein as standard. Melting temperatures and decomposition temperatures were recorded on an OptiMelt MPA-100 apparatus.

Materials: The following reagents were used as received: $N, N$-diisopropylethylamine (TCI), anhydrous dimethylsulfoxide (DMSO; Sigma), cesium fluoride (Sigma), 2,3-dichloro-5,6-dicyano- $p$ benzoquinone (DDQ; TCI), 2-pyridinecarbonitrile (Sigma), isobutyronitrile (Sigma), 2aminobenzonitrile (Alfa Aesar), cyclocarbonitrile (Sigma), o-tolunitrile (Alfa Aeser), 2,4,6trimethylphenylacetonitrile (Alfa Aeser), diphenylacetonitrile (Alfa Aeser), 2-thiophenecarbonitrile (Sigma), 3,5-difluorobenzonitrile (Apollo), 4-(trifluoromethyl)benzonitrile (Apollo), 1cyanonaphthalene (Sigma), 2-chlorobenzonitrile (Merk), 2-chloro-4-fluorobenzene (Sigma), and 2chloro-5-(trifluoromrthyl)benzonitrile (Sigma). The following reagent was prepared according to literature procedure: 2 - $t$-butyl-8-hydroisoquinolino[4,3,2-de]phenanthridin-9-ium chloride. ${ }^{2}$

(1) W. C. Still, M. Kahn, A. Mitra, J. Org. Chem. 1978, 43, 2923-2925.

(2) D. G. Van Greunen, W. Cordier, M. Nell, C. van der Westhuyzen, V. Steenkamp, J. L. Panayides, D. L. Riley, Eur. J. Med. Chem. 2017, 127, 671-690. 


\section{Synthesis of Compounds:}

\section{General Procedures for 1,3-Dipolar Cycloaddition:}

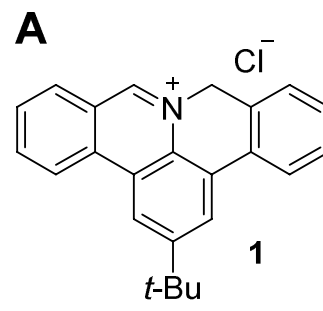

B

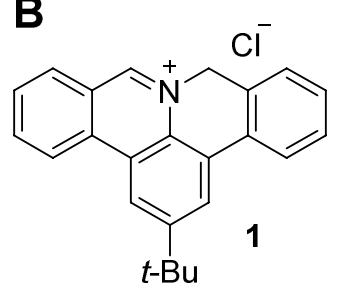

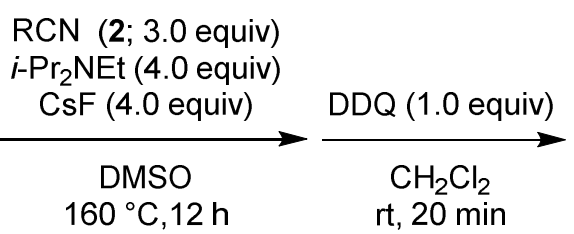

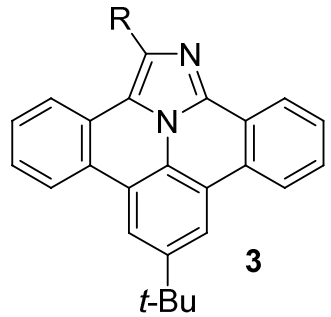

3

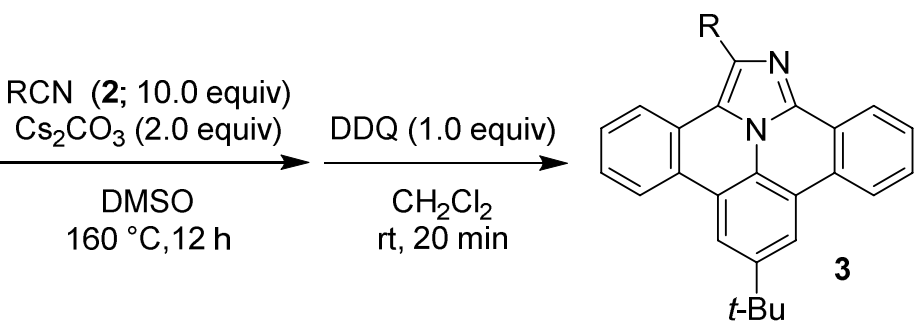

General Procedure A: In a 25-mL Schlenk tube were added iminium salt 1 (36 mg, $0.10 \mathrm{mmol})$ and nitrile $2(0.30 \mathrm{mmol})$, and a magnetic stirring bar. Then, the Schlenk tube was put in a Glove box and $\mathrm{CsF}(61 \mathrm{mg}, 0.40 \mathrm{mmol})$ was added to the mixture. After the Shlenk tube was out from the Glove box, DMSO $(3.0 \mathrm{~mL})$ and $i$ - $\operatorname{Pr}_{2} \mathrm{NEt}(70 \mu \mathrm{L}, 0.40 \mathrm{mmol})$ were added. After stirred for $5 \mathrm{~min}$ at room temperature, the solution was stirred at $160{ }^{\circ} \mathrm{C}$ (oil bath temperature) for $12 \mathrm{~h}$. After cooling to room temperature and dilution with toluene $(50 \mathrm{~mL})$, the mixture was washed with water $(15 \mathrm{~mL})$ four times and brine $(15 \mathrm{~mL})$, then dried over sodium sulfate. After filtration and evaporation, the crude mixture was dissolved in $\mathrm{CH}_{2} \mathrm{Cl}_{2}$ (ca. $5 \mathrm{~mL}$ ), and DDQ (23 mg, $0.10 \mathrm{mmol}$ ) was added. The solution was stirred for $20 \mathrm{~min}$ at room temperature. After dilution with $\mathrm{CH}_{2} \mathrm{Cl}_{2}(50 \mathrm{~mL})$, the mixture was washed with saturated $\mathrm{NaHCO}_{3}$ solution $(15 \mathrm{~mL})$ two times and brine $(15 \mathrm{~mL})$, then dried over $\mathrm{Na}_{2} \mathrm{SO}_{4}$. After filtration and evaporation, the crude product was purified by silica-gel column chromatography.

General Procedure B: In a 25-mL Schlenk tube were added iminium salt 1 (54 $\mathrm{mg}, 0.15 \mathrm{mmol}$ ) and nitrile $2(1.50 \mathrm{mmol})$, and a magnetic stirring bar. Then, the Schlenk tube was put in a Glove box and $\mathrm{Cs}_{2} \mathrm{CO}_{3}(98 \mathrm{mg}, 0.30 \mathrm{mmol}$ ) was added to the mixture. After the Schlenk tube was out from the Glove box, DMSO $(5.0 \mathrm{~mL})$ was added. After stirred for $5 \mathrm{~min}$ at room temperature, the solution was stirred at $160{ }^{\circ} \mathrm{C}$ (oil bath temperature) for $12 \mathrm{~h}$. After cooling to room temperature and dilution with toluene $(50 \mathrm{~mL})$, the mixture was washed with water $(15 \mathrm{~mL})$ four times and brine $(15 \mathrm{~mL})$, then dried over $\mathrm{Na}_{2} \mathrm{SO}_{4}$. After filtration and evaporation, the crude mixture was dissolved in $\mathrm{CH}_{2} \mathrm{Cl}_{2}$ (ca. $5 \mathrm{~mL}$ ), and DDQ (34 mg, $0.15 \mathrm{mmol}$ ) was added. The solution was stirred for $20 \mathrm{~min}$ at room temperature. After dilution with $\mathrm{CH}_{2} \mathrm{Cl}_{2}(50 \mathrm{~mL})$, the mixture was washed with saturated $\mathrm{NaHCO}_{3}$ solution $(15 \mathrm{~mL})$ two times and brine $(15 \mathrm{~mL})$, then dried over $\mathrm{Na}_{2} \mathrm{SO}_{4}$. After filtration and evaporation, the crude product was purified by silica-gel column chromatography.

An Experimental Procedure for 3f on 1.0 mmol Scale: In a 50-mL Schlenk tube were added iminium salt 1 (360 $\mathrm{mg}, 1.0 \mathrm{mmol})$ and nitrile $2 \mathrm{f}(1.17 \mathrm{~g}, 10 \mathrm{mmol})$, and a magnetic stirring bar. Then, the Schlenk tube was put in a Glove box and CsF $(0.61 \mathrm{~g}, 4.0 \mathrm{mmol})$ was added to the mixture. After the Shlenk tube was out from the Glove box, DMSO $(10 \mathrm{~mL})$ and $i-\operatorname{Pr}_{2} \mathrm{NEt}(0.70 \mathrm{~mL}, 4.0$ mmol) were added. After stirred for $5 \mathrm{~min}$ at room temperature, the solution was stirred at $160{ }^{\circ} \mathrm{C}$ (oil bath temperature) for $24 \mathrm{~h}$. After cooling to room temperature and dilution with toluene (100 
$\mathrm{mL})$, the mixture was washed with water $(25 \mathrm{~mL})$ four times and brine $(15 \mathrm{~mL})$, then dried over $\mathrm{Na}_{2} \mathrm{SO}_{4}$. After filtration and evaporation, the crude mixture was dissolved in $\mathrm{CH}_{2} \mathrm{Cl}_{2}(10 \mathrm{~mL})$, and DDQ (227 mg, $1.0 \mathrm{mmol}$ ) was added. The solution was stirred for $20 \mathrm{~min}$ at room temperature. After dilution with $\mathrm{CH}_{2} \mathrm{Cl}_{2}(50 \mathrm{~mL})$, the mixture was washed with saturated $\mathrm{NaHCO}_{3}$ solution $(15 \mathrm{~mL})$ two times and brine $(15 \mathrm{~mL})$, then dried over $\mathrm{Na}_{2} \mathrm{SO}_{4}$. After filtration and evaporation, the crude product was purified by silica-gel column chromatography to yield $\mathbf{3 f}$ as a yellow solid (135 $\mathrm{mg}$, $0.31 \mathrm{mmol}, 31 \%)$.

Table S1. Complete Table for Optimization of Reaction Conditions.

\begin{tabular}{|c|c|c|c|c|c|}
\hline entry & base & additive & $\begin{array}{c}\text { temperature } \\
\left({ }^{\circ} \mathrm{C}\right)\end{array}$ & $\begin{array}{c}\text { NMR yield } \\
(\%)\end{array}$ & note \\
\hline 1 & $i-\mathrm{Pr}_{2} \mathrm{NEt}(2.0$ equiv) & --- & 160 & 0 & \multirow{2}{*}{ effect of CsF } \\
\hline 2 & $i-\mathrm{Pr}_{2} \mathrm{NEt}$ (2.0 equiv) & CsF (4.0 equiv) & 160 & 48 & \\
\hline S1 & $i-\mathrm{Pr}_{2} \mathrm{NEt}$ (2.0 equiv) & CsF (4.0 equiv) & 140 & 30 & \multirow{2}{*}{$\begin{array}{l}\text { screening of } \\
\text { temperature }\end{array}$} \\
\hline $\mathrm{S} 2$ & $i-\mathrm{Pr}_{2} \mathrm{NEt}(2.0$ equiv) & CsF (4.0 equiv) & 180 & 48 & \\
\hline 3 & $i-\mathrm{Pr}_{2} \mathrm{NEt}$ (4.0 equiv) & CsF (4.0 equiv) & 160 & 52 & \multirow{3}{*}{$\begin{array}{l}\text { screening of } \\
\text { CsF amount }\end{array}$} \\
\hline S3 & $i-\mathrm{Pr}_{2} \mathrm{NEt}$ (4.0 equiv) & CsF (2.0 equiv) & 160 & 12 & \\
\hline S4 & $i-\mathrm{Pr}_{2} \mathrm{NEt}$ (4.0 equiv) & CsF (6.0 equiv) & 160 & 47 & \\
\hline S5 & $i-\mathrm{Pr}_{2} \mathrm{NEt}$ (4.0 equiv) & KF (4.0 equiv) & 160 & 13 & \multirow{6}{*}{$\begin{array}{l}\text { screening of } \\
\text { additive }\end{array}$} \\
\hline 4 & $i-\mathrm{Pr}_{2} \mathrm{NEt}$ (4.0 equiv) & $\mathrm{NaF}$ (4.0 equiv) & 160 & 5 & \\
\hline S6 & $i-\mathrm{Pr}_{2} \mathrm{NEt}$ (4.0 equiv) & LiF (4.0 equiv) & 160 & 6 & \\
\hline $\mathrm{S} 7$ & $i-\mathrm{Pr}_{2} \mathrm{NEt}$ (4.0 equiv) & $\mathrm{B}\left(\mathrm{C}_{6} \mathrm{~F}_{5}\right)_{3}$ (3.0 equiv) & 160 & 0 & \\
\hline 5 & $i-\mathrm{Pr}_{2} \mathrm{NEt}$ (4.0 equiv) & CsOAc (4.0 equiv) & 160 & 30 & \\
\hline 6 & $i-\mathrm{Pr}_{2} \mathrm{NEt}$ (4.0 equiv) & $\begin{array}{c}\text { CsF (4.0 equiv) } \\
\text { 18-crown-6 ( } 6.0 \text { equiv) }\end{array}$ & 160 & 0 & \\
\hline 7 & $\mathrm{Cs}_{2} \mathrm{CO}_{3}(4.0$ equiv) & --- & 160 & 47 & \multirow{2}{*}{$\begin{array}{l}\text { screening of } \\
\text { base }\end{array}$} \\
\hline S8 & $t$-BuOK (1.0 equiv) & CsF (4.0 equiv) & 160 & 53 & \\
\hline
\end{tabular}

Note:

1) Additive: Cesium ion is essential to obtain high yield. The order of reactivity is cesium $>$ potassium $>$ sodium $\sim$ lithium (compare entries 3 and 4; S5 and S6).

2) Amount of Additive: The amount of 4.0 equivalents to the substrate is necessary (compare entries 3, S3, and S4).

3) Anion: Counter anions have a marginal effect on the reactivity (compare entries 3, 5, and 7).

4) Temperature: $160^{\circ} \mathrm{C}$ is best (compare entries $2, \mathrm{~S} 1$, and S2). 


\section{8-tert-butyl-2-(2-chlorophenyl)tribenzo[b,g,ij]imidazo[2,1,5-de]quinolizine (3a):}

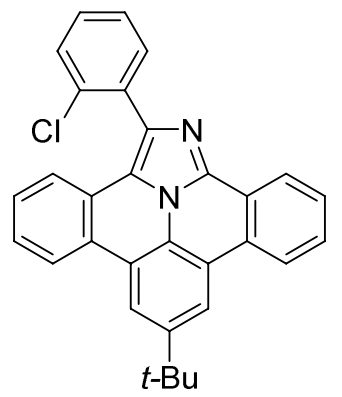

Using procedure A, 3a (23.9 $\mathrm{mg}, 0.052 \mathrm{mmol}, 52 \%)$ was obtained from 2a (41 $\mathrm{mg}, 0.30 \mathrm{mmol}$ ); Colorless solid; $R_{\mathrm{f}}: 0.3$ (hexane:ethyl acetate $=10: 1$ ); $\mathrm{mp}$ $328-330{ }^{\circ} \mathrm{C}$; IR (neat); $\mathrm{cm}^{-1} 2950,2905,2866,1732,1614,1572,1528,1491$, $1458,1442,1413,1388,1361,1337,1247,1203,1121,1041,963,944,864$, 783, 761, 751, 740, 729, 698; ${ }^{1} \mathrm{H}$ NMR (400 MHz, $\left.\mathrm{CDCl}_{3}, 300 \mathrm{~K}\right) \delta 8.80-8.70$ $(\mathrm{m}, 1 \mathrm{H}), 8.52-8.45(\mathrm{~m}, 2 \mathrm{H}), 8.43(\mathrm{~s}, 1 \mathrm{H}), 8.38(\mathrm{~d}, J=8.0 \mathrm{~Hz}, 1 \mathrm{H}), 7.74(\mathrm{dd}, J$ $=5.6,3.6 \mathrm{~Hz}, 1 \mathrm{H}), 7.70-7.63(\mathrm{~m}, 2 \mathrm{H}), 7.62(\mathrm{dd}, J=5.6,4.0 \mathrm{~Hz}, 1 \mathrm{H}), 7.56(\mathrm{~d}, J$

$=8.0 \mathrm{~Hz}, 1 \mathrm{H}), 7.53-7.44(\mathrm{~m}, 3 \mathrm{H}), 7.35(\mathrm{t}, J=7.6 \mathrm{~Hz}, 1 \mathrm{H}), 1.58(\mathrm{~s}, 9 \mathrm{H}) ;{ }^{13} \mathrm{C}$

NMR $\left(100 \mathrm{MHz}, \mathrm{CDCl}_{3}, 300 \mathrm{~K}\right) \delta 147.9,137.2,135.4,135.1,134.5,133.0,129.9,129.9,128.7$, $128.6,128.5,128.0,127.2,127.1,126.6,126.4,125.4,124.2,123.9,123.8,123.0,122.8,122.8$, 121.9, 120.8, 118.2, 118.0, 35.5, 31.8 (3C); HRMS (ESI) $m / z$ calcd for $\mathrm{C}_{31} \mathrm{H}_{24} \mathrm{ClN}_{2}[\mathrm{M}+\mathrm{H}]^{+}$ 459.1628 , found 459.1629 .

\section{2-([1,1'-biphenyl]-4-yl)-8-tert-butyltribenzo $[b, g, i j]$ imidazo[2,1,5-de]quinolizine (3b):}

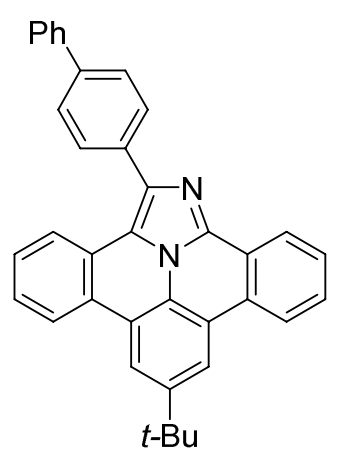

Using procedure $\mathbf{A}, \mathbf{3 b}(22.7 \mathrm{mg}, 0.045 \mathrm{mmol}, 45 \%)$ was obtained from $\mathbf{2 b}$ (54 $\mathrm{mg}, 0.30 \mathrm{mmol}$ ); Colorless solid; $R_{\mathrm{f}}: 0.3$ (hexane:dichloromethane $=4: 6$ ); $\mathrm{mp}$ 331-333 ${ }^{\circ} \mathrm{C}$ (decomp); IR (neat) $\mathrm{cm}^{-1}$ 2960, 2922, 2849, 1611, 1571, 1528, $1491,1473,1443,1414,1385,1360,1335,1275,1261,1202,1092,1042$, $1008,963,910,866,839,784,763,749,727,695,663 ;{ }^{1} \mathrm{H}$ NMR (400 MHz, $\left.\mathrm{CDCl}_{3}, 300 \mathrm{~K}\right) \delta 8.83-8.76(\mathrm{~m}, 1 \mathrm{H}), 8.54-8.47(\mathrm{~m}, 2 \mathrm{H}), 8.42(\mathrm{~s}, 1 \mathrm{H}), 8.39(\mathrm{~d}, J$ $=8.0 \mathrm{~Hz}, 1 \mathrm{H}), 8.25(\mathrm{~d}, J=8.4 \mathrm{~Hz}, 1 \mathrm{H}), 7.96(\mathrm{~d}, J=8.0 \mathrm{~Hz}, 2 \mathrm{H}), 7.81(\mathrm{~d}, J=$ $8.0 \mathrm{~Hz}, 2 \mathrm{H}), 7.74(\mathrm{~d}, J=7.2 \mathrm{~Hz}, 2 \mathrm{H}), 7.72-7.65(\mathrm{~m}, 2 \mathrm{H}), 7.50(\mathrm{t}, J=7.6 \mathrm{~Hz}$, $1 \mathrm{H}), 7.39$ (t, $J=7.6 \mathrm{~Hz}, 2 \mathrm{H}), 1.59$ (s, 9H); ${ }^{13} \mathrm{C} \mathrm{NMR}\left(100 \mathrm{MHz}, \mathrm{CDCl}_{3}, 300 \mathrm{~K}\right)$ $\delta 147.9,141.0,140.8,137.8,137.4,135.5,135.3,130.4(2 \mathrm{C}), 128.9(2 \mathrm{C}), 128.7,128.5,128.0,127.4$ (2C), 127.2 (2C), 127.2, 127.1, 126.7, 126.5, 125.7, 124.3, 124.2, 123.7, 123.7, 123.3, 122.8, 121.9, 119.7, 118.2, 118.0, 35.5, 31.9 (3C); HRMS (ESI) $m / z$ calcd for $\mathrm{C}_{37} \mathrm{H}_{29} \mathrm{~N}_{2}[\mathrm{M}+\mathrm{H}]^{+}$501.2331, found 501.2335 .

\section{2-(benzo[d][1,3]dioxol-5-yl)-8-tert-butyltribenzo[b,g,ij] $]$ imidazo[2,1,5-de]quinolizine (3c):}

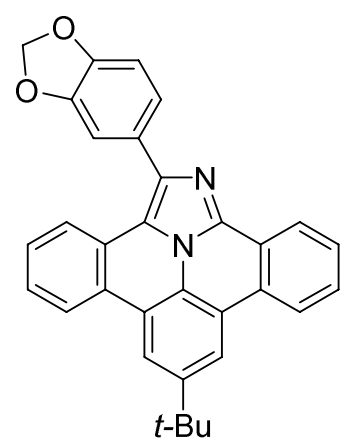

Using procedure A, 3c (21.4 mg, $0.046 \mathrm{mmol}, 46 \%)$ was obtained from $2 \mathbf{c}$ (44 $\mathrm{mg}, 0.30 \mathrm{mmol}$ ); Colorless solid; $R_{\mathrm{f}}: 0.3$ (hexane:dichloromethane $=1: 9$ ); $119-123{ }^{\circ} \mathrm{C}$ (decomp); IR (neat) $\mathrm{cm}^{-1}$ 2956, 2905, 2869, 1731, 1614, 1572, $1527,1505,1445,1414,1431,1363,1327,1291,1232,1202,1103,1038,971$, $930,884,865,830,814,784,766,743,729,709,697,683,662 ;{ }^{1} \mathrm{H}$ NMR $\left(400 \mathrm{MHz}, \mathrm{CDCl}_{3}, 300 \mathrm{~K}\right) \delta 8.79-8.71(\mathrm{~m}, 1 \mathrm{H}), 8.51-8.44(\mathrm{~m}, 2 \mathrm{H}), 8.40(\mathrm{~d}, J$ $=1.3 \mathrm{~Hz}, 1 \mathrm{H}), 8.36(\mathrm{~d}, J=8.4 \mathrm{~Hz}, 1 \mathrm{H}), 8.16(\mathrm{~d}, J=7.2 \mathrm{~Hz}, 1 \mathrm{H}), 7.71-7.63(\mathrm{~m}$, 2H), $7.48(\mathrm{t}, J=7.2 \mathrm{~Hz}, 1 \mathrm{H}), 7.38(\mathrm{dt}, J=8.0,0.8 \mathrm{~Hz}, 1 \mathrm{H}), 7.35-7.30(\mathrm{~m}, 2 \mathrm{H})$, $7.00(\mathrm{~d}, J=7.6 \mathrm{~Hz}, 1 \mathrm{H}), 6.08(\mathrm{~s}, 2 \mathrm{H}), 1.58(\mathrm{~s}, 9 \mathrm{H}) ;{ }^{13} \mathrm{C} \mathrm{NMR}(100 \mathrm{MHz}$, $\left.\mathrm{CDCl}_{3}, 300 \mathrm{~K}\right) \delta 147.9,147.8,147.7,137.9,137.1,130.2,128.7,128.5,128.4,128.0,127.1,126.6$, $126.5,125.8,124.2,123.8,123.8,123.6,123.3,122.8,121.9,119.4,118.2,117.9,110.6,108.6$, 101.2, 35.5, 31.8 (3C); HRMS (ESI) $m / z$ calcd for $\mathrm{C}_{32} \mathrm{H}_{25} \mathrm{~N}_{2} \mathrm{O}_{2}[\mathrm{M}+\mathrm{H}]^{+} 469.1916$, found 469.1914. 


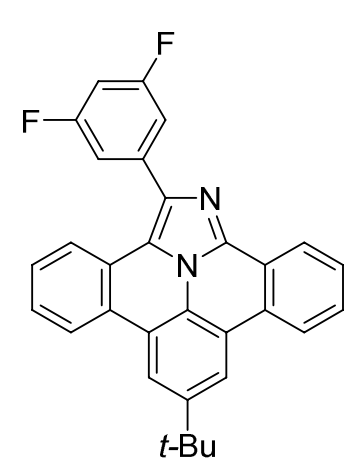

Using procedure A, 3d (32.1 mg, $0.070 \mathrm{mmol}, 70 \%)$ was obtained from $2 \mathbf{d}$ (42 $\mathrm{mg}, 0.30 \mathrm{mmol}$ ); Colorless solid; $R_{\mathrm{f}}: 0.4$ (hexane:dichloromethane $=6: 4$ ); $\mathrm{mp}$ 258-260 ${ }^{\circ} \mathrm{C}$ (decomp); IR (neat) $\mathrm{cm}^{-1}$ 3093, 2962, 2909, 2869, 1624, 1589, $1573,1526,1492,1454,1443,1431,1414,1390,1365,1301,1245,1118,984$, 858, 831, 784, 739, 697, 676, 662; ${ }^{1} \mathrm{H}$ NMR (400 MHz, $\left.\mathrm{CDCl}_{3}, 300 \mathrm{~K}\right) \delta$ 8.74-8.68 (m, 1H), 8.52-8.44 (m, 2H), $8.42(\mathrm{~s}, 1 \mathrm{H}), 8.38(\mathrm{~d}, J=8.4 \mathrm{~Hz}, 1 \mathrm{H})$, $8.13(\mathrm{~d}, J=7.2 \mathrm{~Hz}, 1 \mathrm{H}), 7.72-7.63(\mathrm{~m}, 2 \mathrm{H}), 7.53(\mathrm{t}, J=8.1 \mathrm{~Hz}, 1 \mathrm{H}), 7.48-$ $7.38(\mathrm{~m}, 3 \mathrm{H}), 6.97-6.88(\mathrm{~m}, 1 \mathrm{H}), 1.59(\mathrm{~s}, 9 \mathrm{H}) ;{ }^{13} \mathrm{C} \mathrm{NMR}\left(100 \mathrm{MHz}, \mathrm{CDCl}_{3}\right.$, $300 \mathrm{~K}) \delta 163.2(\mathrm{dd}, J=248,13.1 \mathrm{~Hz}, 2 \mathrm{C}), 148.1,139.8,139.7,139.6,137.5$, $135.6,128.8,128.8,128.6,128.1,127.6,126.8,126.2,125.0,124.2,123.5(\mathrm{t}, J=3.1 \mathrm{~Hz}), 122.8$, 122.7, 121.9, 120.0, 118.4, 118.1, 113.0 (dd, $J=18.5,7.1 \mathrm{~Hz}), 103.5$ (t, $J=25.4 \mathrm{~Hz}, 2 \mathrm{C}), 35.6,31.8$ (3C); HRMS (ESI) $m / z$ calcd for $\mathrm{C}_{31} \mathrm{H}_{23} \mathrm{~F}_{2} \mathrm{~N}_{2}[\mathrm{M}+\mathrm{H}]^{+} 461.1829$, found 461.1822 .

\section{8-tert-butyl-2-(4-(trifluoromethyl)phenyl)tribenzo[b,g,ij] $]$ imidazo[2,1,5-de]quinolizine (3e):}

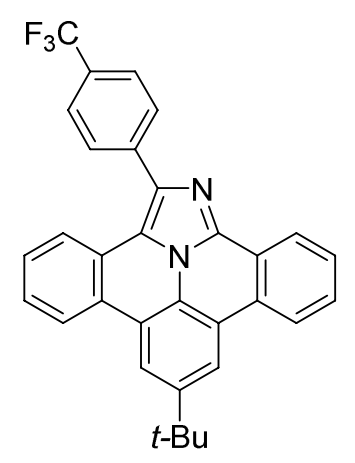

Using procedure A, 3e (28.9 mg, $0.059 \mathrm{mmol}, 59 \%)$ was obtained from 2e (51 $\mathrm{mg}, 0.30 \mathrm{mmol}$ ); Colorless solid; $R_{\mathrm{f}}: 0.3$ (hexane:dichloromethane $=6: 4$ ); $\mathrm{mp}$ 333-338 ${ }^{\circ} \mathrm{C}$ (decomp); IR (neat) $\mathrm{cm}^{-1}$ 2966, 2925, 2869, 1619, 1571, 1526, $1492,1478,1444,1414,1328,1250,1157,1103,1071,1059,1016,963,861$, 842, 784, 761, 698, 687; ${ }^{1} \mathrm{H}$ NMR $\left(400 \mathrm{MHz}, \mathrm{CDCl}_{3}, 300 \mathrm{~K}\right) \delta 8.78-8.71(\mathrm{~m}$, $1 \mathrm{H}), 8.54-8.44(\mathrm{~m}, 2 \mathrm{H}), 8.43(\mathrm{~s}, 1 \mathrm{H}), 8.40(\mathrm{~d}, J=8.0 \mathrm{~Hz}, 1 \mathrm{H}), 8.10(\mathrm{~d}, J=8.0$ $\mathrm{Hz}, 1 \mathrm{H}), 8.03(\mathrm{~d}, J=8.0 \mathrm{~Hz}, 2 \mathrm{H}), 7.82(\mathrm{~d}, J=7.6 \mathrm{~Hz}, 2 \mathrm{H}), 7.74-7.65(\mathrm{~m}, 2 \mathrm{H})$, $7.53(\mathrm{t}, J=7.6 \mathrm{~Hz}, 1 \mathrm{H}), 7.40(\mathrm{t}, J=7.6 \mathrm{~Hz}, 1 \mathrm{H}), 1.59(\mathrm{~s}, 9 \mathrm{H}) ;{ }^{13} \mathrm{C} \mathrm{NMR}(100$ $\left.\mathrm{MHz}, \mathrm{CDCl}_{3}, 300 \mathrm{~K}\right) \delta 148.1,140.3,137.7,136.6,130.3,130.2,129.9,128.8$, 128.7, 128.6, 128.1, 127.5, 126.9, 126.4 (2C), 125.6 (q, $J=3.7 \mathrm{~Hz}), 125.3(2 \mathrm{C}), 124.2,123.7,123.5$, 123.4, 122.9, 122.8, 122.0, 120.1, 118.4, 118.1, 35.5, 31.8 (3C); HRMS (ESI) $\mathrm{m} / z$ calcd for $\mathrm{C}_{32} \mathrm{H}_{24} \mathrm{~F}_{3} \mathrm{~N}_{2}[\mathrm{M}+\mathrm{H}]^{+}$493.1892, found 493.1900.

\section{8-tert-butyl-2-(o-tolyl)tribenzo[b,g,ij]imidazo[2,1,5-de]quinolizine (3f):}

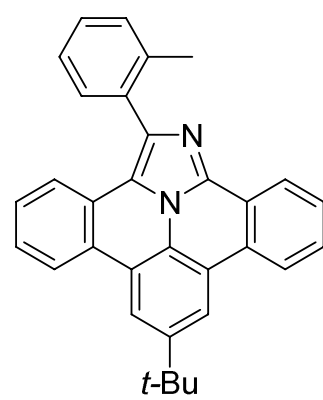

Using procedure A, $3 \mathbf{3 f}(14.2 \mathrm{mg}, 0.032 \mathrm{mmol}, 32 \%)$ was obtained from $2 \mathbf{f}$ (35 $\mathrm{mg}, 0.30 \mathrm{mmol}$ ); Colorless solid; $R_{\mathrm{f}}$ : 0.3 (hexane:dichloromethane $=4: 6$ ); $\mathrm{mp}$ 260-264 ${ }^{\circ} \mathrm{C}$ (decomp); IR (neat) $\mathrm{cm}^{-1}$ 2950, 2918, 2850, 1729, 1612, 1572, $1528,1491,1464,1443,1413,1386,1360,1291,1246,1120,1106,1093,1040$, 961, 946, 862, 808, 783, 742, 728, 699, 680, 654; ${ }^{1} \mathrm{H}$ NMR (400 MHz, $\mathrm{CDCl}_{3}$, $300 \mathrm{~K}) \delta 8.81-8.73(\mathrm{~m}, 1 \mathrm{H}), 8.54-8.46(\mathrm{~m}, 2 \mathrm{H}), 8.43(\mathrm{~s}, 1 \mathrm{H}), 8.37(\mathrm{~d}, J=8.0$ $\mathrm{Hz}, 1 \mathrm{H}), 7.72-7.64(\mathrm{~m}, 2 \mathrm{H}), 7.59(\mathrm{~d}, J=7.2 \mathrm{~Hz}, 1 \mathrm{H}), 7.55(\mathrm{~d}, J=8.0 \mathrm{~Hz}, 1 \mathrm{H})$, 7.50-7.28 (m, 5H), $2.26(\mathrm{~s}, 3 \mathrm{H}), 1.59(\mathrm{~s}, 9 \mathrm{H}) ;{ }^{13} \mathrm{C} \mathrm{NMR}\left(100 \mathrm{MHz}, \mathrm{CDCl}_{3}, 300\right.$ K) $\delta 147.8,138.0,137.2,137.1,135.6,131.1,130.4,128.8,128.7,128.6,128.4,128.0,126.9,126.5$, $126.5,126.2$, 125.8, 124.2, 123.9, 123.4, 123.1, 122.9, 122.8, 121.9, 120.0, 118.1, 118.0, 35.5, 31.9 (3C), 20.0; HRMS (ESI) $m / z$ calcd for $\mathrm{C}_{32} \mathrm{H}_{27} \mathrm{~N}_{2}[\mathrm{M}+\mathrm{H}]^{+} 439.2174$, found 439.2169. 


\section{2-(8-tert-butyltribenzo[b,g,ij] imidazo[2,1,5-de]quinolizin-2-yl)aniline (3g):}

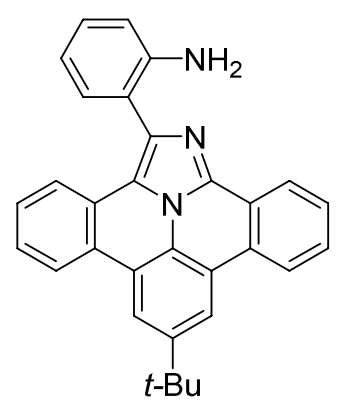

Using procedure A, $\mathbf{3 g}$ (26.9 $\mathrm{mg}, 0.031 \mathrm{mmol}, 31 \%$ ) was obtained from $\mathbf{2 g}$ (71 $\mathrm{mg}, 0.60 \mathrm{mmol}$ ); Yellow solid; $R_{\mathrm{f}}: 0.3$ (hexane:ethyl acetate $=8: 2$ ); $\mathrm{mp} 245$ $249{ }^{\circ} \mathrm{C}$ (decomp); IR (neat) $\mathrm{cm}^{-1} 3464,3405,3046,2949,2924,2854,1729$, $1587,1574,1564,1461,1442,1413,1362,1248,1211,1147,1092,1043,995$, 963, 864, 793, 783, 757, 728, 698, 662; ${ }^{1} \mathrm{H} \mathrm{NMR}\left(400 \mathrm{MHz}, \mathrm{CDCl}_{3}, 295 \mathrm{~K}\right) \delta$ $8.77-8.69(\mathrm{~m}, 1 \mathrm{H}), 8.52-8.45(\mathrm{~m}, 2 \mathrm{H}), 8.42(\mathrm{~d}, J=1.6 \mathrm{~Hz}, 1 \mathrm{H}), 8.36(\mathrm{~d}, J=$ $8.0 \mathrm{~Hz}, 1 \mathrm{H}), 8.03(\mathrm{~d}, J=8.0 \mathrm{~Hz}, 1 \mathrm{H}), 7.70-7.61(\mathrm{~m}, 2 \mathrm{H}), 7.55(\mathrm{~d}, J=7.6 \mathrm{~Hz}$, $1 \mathrm{H}), 7.49(\mathrm{t}, J=8.0 \mathrm{~Hz}, 1 \mathrm{H}), 7.38(\mathrm{t}, J=8.0 \mathrm{~Hz}, 1 \mathrm{H}), 7.30(\mathrm{t}, J=8.0 \mathrm{~Hz}, 1 \mathrm{H})$, 6.96-6.88 (m, 2H), 4.38 (br s, 2H), 1.59 (s, 9H); $\left.{ }^{13} \mathrm{C} \mathrm{NMR} \mathrm{(100} \mathrm{MHz,} \mathrm{CDCl}_{3}, 300 \mathrm{~K}\right) \delta 147.9,145.8$, $137.4,135.2$, 131.6, 129.6, 128.6, 128.6, 128.5, 127.9, 127.1, 126.5, 126.4, 125.5, 124.2, 124.1, 123.6, 123.0, 122.9, 122.8, 121.7, 120.7, 120.2, 118.4, 118.1, 118.0, 116.3, 35.5, 31.9 (3C); HRMS (ESI) $m / z$ calcd for $\mathrm{C}_{31} \mathrm{H}_{26} \mathrm{~N}_{3}[\mathrm{M}+\mathrm{H}]^{+} 440.2127$, found 440.2126 .

\section{8-tert-butyl-2-(naphthalen-1-yl)tribenzo[b,g,ij] $]$ imidazo[2,1,5-de]quinolizine (3h):}

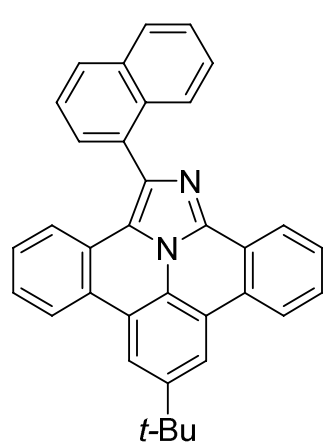

Using procedure A, $\mathbf{3 h}$ (16.9 mg, $0.036 \mathrm{mmol}, 36 \%)$ was obtained from $\mathbf{2 h}$ (46 $\mathrm{mg}, 0.30 \mathrm{mmol}$ ); Light yellow solid; $R_{\mathrm{f}}$ : 0.3 (hexane:dichloromethane $=4: 6$ ); mp 270-272 ${ }^{\circ} \mathrm{C}$ (decomp); IR (neat) $\mathrm{cm}^{-1} 3047,2952,2922,2852,1729,1613$, $1572,1527,1488,1457,1442,1416,1376,1363,1255,1023,1011,946,864$, 804, 777, 729, 707, 697, 680; ${ }^{1} \mathrm{H}$ NMR $\left(400 \mathrm{MHz}, \mathrm{CDCl}_{3}, 300 \mathrm{~K}\right) \delta 8.84-8.78$ $(\mathrm{m}, 1 \mathrm{H}), 8.58-8.51(\mathrm{~m}, 2 \mathrm{H}), 8.47(\mathrm{~s}, 1 \mathrm{H}), 8.40(\mathrm{~d}, J=8.0 \mathrm{~Hz}, 1 \mathrm{H}), 8.07(\mathrm{~d}, J=$ $8.4 \mathrm{~Hz}, 1 \mathrm{H}), 8.01(\mathrm{~d}, J=8.4 \mathrm{~Hz}, 1 \mathrm{H}), 7.89(\mathrm{~d}, J=6.8 \mathrm{~Hz}, 1 \mathrm{H}), 7.84(\mathrm{~d}, J=8.4$ $\mathrm{Hz}, 1 \mathrm{H}), 7.75-7.63(\mathrm{~m}, 3 \mathrm{H}), 7.53(\mathrm{t}, J=7.2 \mathrm{~Hz}, 1 \mathrm{H}), 7.46-7.34(\mathrm{~m}, 3 \mathrm{H}), 7.15$ $(\mathrm{t}, J=7.6 \mathrm{~Hz}, 1 \mathrm{H}), 1.63(\mathrm{~s}, 9 \mathrm{H}) ;{ }^{13} \mathrm{C} \mathrm{NMR}\left(100 \mathrm{MHz}, \mathrm{CDCl}_{3}, 300 \mathrm{~K}\right) \delta 147.9$, $137.4,135.8,134.0,133.7,132.7,130.9,129.0,128.9,128.7,128.5,128.5,128.3,128.0,127.0$, $126.6,126.5,126.5,126.4,126.0,125.7,125.4,124.3,124.1,123.8,123.0,122.9,122.0,121.0$, 118.2 118.0, 35.5, 31.9 (3C); HRMS (ESI) $m / z$ calcd for $\mathrm{C}_{35} \mathrm{H}_{27} \mathrm{~N}_{2}[\mathrm{M}+\mathrm{H}]^{+} 475.2174$, found 475.2172 .

\section{8-tert-butyl-2-(2-chloro-4-fluorophenyl)tribenzo[b,g,ij]imidazo[2,1,5-de]quinolizine (3i):}

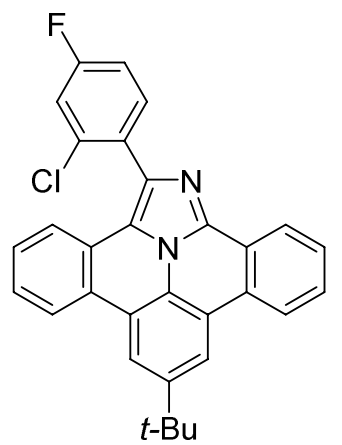

Using procedure B, $3 \mathbf{i}(28.7 \mathrm{mg}, 0.060 \mathrm{mmol}, 40 \%)$ was obtained from $\mathbf{2 i}$ (233 $\mathrm{mg}, 1.50 \mathrm{mmol}$ ); Colorless solid; $R_{\mathrm{f}}: 0.3$ (hexane:ethyl acetate $=10: 1$ ); $\mathrm{mp}$ 317-320 ${ }^{\circ} \mathrm{C}$; IR (neat); $\mathrm{cm}^{-1} 3071,2951,2905,2867,1606,1572,1530,1468$, $1442,1414,1388,1362,1257,1216,1121,1038,965,895,863,821,783,759$, 729, 698, 687; ${ }^{1} \mathrm{H}$ NMR (400 MHz, $\left.\mathrm{CDCl}_{3}, 300 \mathrm{~K}\right) \delta 8.77$ (br s, $\left.1 \mathrm{H}\right), 8.54-8.46$ $(\mathrm{m}, 2 \mathrm{H}), 8.44(\mathrm{~s}, 1 \mathrm{H}), 8.40(\mathrm{~d}, J=8.0 \mathrm{~Hz}, 1 \mathrm{H}), 7.78-7.63(\mathrm{~m}, 3 \mathrm{H}), 7.56-7.48$ $(\mathrm{m}, 2 \mathrm{H}), 7.42-7.34(\mathrm{~m}, 2 \mathrm{H}), 7.20(\mathrm{t}, J=6.8 \mathrm{~Hz}, 1 \mathrm{H}), 1.59(\mathrm{~s}, 9 \mathrm{H}) ;{ }^{13} \mathrm{C} \mathrm{NMR}$ $\left(126 \mathrm{MHz}, \mathrm{CDCl}_{3}, 300 \mathrm{~K}\right) \delta 163.8,161.8,148.2,137.1,136.0(\mathrm{~d}, J=10 \mathrm{~Hz})$, $134.1(\mathrm{~d}, J=8.6 \mathrm{~Hz}), 128.8,128.7,128.6,128.1,127.4,126.7,126.2,125.1$, $124.3,123.7,123.6,123.2,123.1,122.9,122.8,122.0,120.9,118.3,118.2,117.3(\mathrm{~d}, J=24 \mathrm{~Hz})$, $114.6(\mathrm{~d}, J=21 \mathrm{~Hz}), 35.5,31.8(3 \mathrm{C})$; HRMS (ESI) $m / z$ calcd for $\mathrm{C}_{31} \mathrm{H}_{23} \mathrm{ClFN}_{2}[\mathrm{M}+\mathrm{H}]^{+} 477.1534$, found 477.1542 . 
(3j):

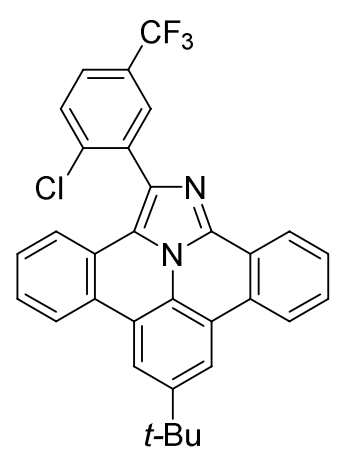

Using procedure B, 3j (50 mg, $0.094 \mathrm{mmol}, 63 \%$ ) was obtained from $\mathbf{2 j}$ (308 $\mathrm{mg}, 1.50 \mathrm{mmol}$ ); Colorless solid; $R_{\mathrm{f}}: 0.3$ (hexane:ethyl acetate $=30: 1$ ); $\mathrm{mp}$ 259-270 ${ }^{\circ} \mathrm{C}$; IR (neat); $\mathrm{cm}^{-1} 3032,2959,2913,2870,1614,1573,1527,1478$, $1444,1409,1377,1339,1318,1292,1265,1168,1114,1084,1071,1041,973$, 940, 905, 866, 845, 825, 783, 729, 696; ${ }^{1} \mathrm{H}$ NMR (400 MHz, $\left.\mathrm{CDCl}_{3}, 300 \mathrm{~K}\right) \delta$ 8.76-8.68 (m, 1H), 8.53-8.46 (m, 2H), $8.45(\mathrm{~s}, 1 \mathrm{H}), 8.40(\mathrm{~d}, J=8.0 \mathrm{~Hz}, 1 \mathrm{H})$, $8.01(\mathrm{~s}, 1 \mathrm{H}), 7.78-7.64(\mathrm{~m}, 4 \mathrm{H}), 7.56-7.46(\mathrm{~m}, 2 \mathrm{H}), 7.39(\mathrm{t}, J=7.6 \mathrm{~Hz}, 1 \mathrm{H})$, $1.59(\mathrm{~s}, 9 \mathrm{H}) ;{ }^{13} \mathrm{C}$ NMR $\left(100 \mathrm{MHz}, \mathrm{CDCl}_{3}, 300 \mathrm{~K}\right) \delta 148.2,138.9,137.5,136.3$, $132.7,130.5,130.2,130.1(\mathrm{q}, J=3.6 \mathrm{~Hz}), 129.7(\mathrm{q}, J=33 \mathrm{~Hz}), 128.8,128.7$, 128.7, 128.1, 127.5, 126.8, 126.6 (q, $J=3.5 \mathrm{~Hz}), 126.2,125.1,124.9,124.2,123.8,123.6,123.2$, $122.9,122.7,122.0,121.2,118.4,118.2,35.5,31.8$ (3C); HRMS (ESI) $m / z$ calcd for $\mathrm{C}_{32} \mathrm{H}_{23} \mathrm{ClF}_{3} \mathrm{~N}_{2}$ $[\mathrm{M}+\mathrm{H}]^{+} 527.1502$, found 527.1509 .

\section{8-tert-butyl-2-(2-chloropyridin-4-yl)tribenzo[b,g,ij]imidazo[2,1,5-de]quinolizine (3k):}

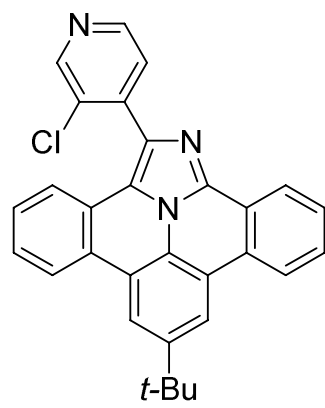

Using procedure B, 3k (30 mg, $0.064 \mathrm{mmol}, 43 \%$ ) was obtained from 2k (208 $\mathrm{mg}, 1.50 \mathrm{mmol}$ ); Colorless solid; $R_{\mathrm{f}}: 0.3$ (hexane:ethyl acetate $=2: 1$ ); mp 353$354{ }^{\circ} \mathrm{C}$; IR (neat); $\mathrm{cm}^{-1} 3356,3071,2959,2909,2861,2226,1954,1732,1588$, $1573,1532,1492,1460,1442,1413,1398,1362,1338,1271,1250,1205$, $1169,1153,1133,1122,1102,1090,1033,967,921,864,842,784,761,729$, 699, 680, 655; ${ }^{1} \mathrm{H}$ NMR (400 MHz, $\left.\mathrm{CDCl}_{3}, 300 \mathrm{~K}\right) \delta 8.84(\mathrm{~s}, 1 \mathrm{H}), 8.75-8.67$ $(\mathrm{m}, 2 \mathrm{H}), 8.55-8.48(\mathrm{~m}, 2 \mathrm{H}), 8.46(\mathrm{~s}, 1 \mathrm{H}), 8.42(\mathrm{~d}, J=8.0 \mathrm{~Hz}, 1 \mathrm{H}), 7.76-7.65$ $(\mathrm{m}, 3 \mathrm{H}), 7.59-7.51(\mathrm{~m}, 2 \mathrm{H}), 7.42(\mathrm{t}, J=7.2 \mathrm{~Hz}, 1 \mathrm{H}), 1.60(\mathrm{~s}, 9 \mathrm{H}) ;{ }^{13} \mathrm{C} \mathrm{NMR}$ $\left(100 \mathrm{MHz}, \mathrm{CDCl}_{3}, 300 \mathrm{~K}\right) \delta 150.1,148.3,148.0,143.5,143.4,137.8,132.5,131.4,128.9,128.7$, $128.2,127.8,127.1,126.9,126.1,124.7,124.2,124.0,123.6,123.2,122.9,122.7,122.0,121.4$, 118.4, 118.2, 35.6, 31.8 (3C); HRMS (ESI) $\mathrm{m} / z$ calcd for $\mathrm{C}_{30} \mathrm{H}_{23} \mathrm{ClN}_{3}[\mathrm{M}+\mathrm{H}]^{+} 460.1581$, found 460.1584 .

\section{8-tert-butyl-2-(pyridin-2-yl)tribenzo[b,g,ij]imidazo[2,1,5-de]quinolizine (31):}

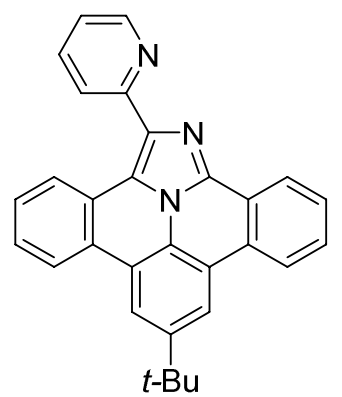

Using procedure A, 31 (17.8 mg, $0.042 \mathrm{mmol}, 42 \%)$ was obtained from $2 \mathbf{l}$ (62 $\mathrm{mg}, 0.60 \mathrm{mmol}$ ); Pink solid; $R_{\mathrm{f}}: 0.3$ (hexane:ethyl acetate $=8: 2$ ); $\mathrm{mp} 260$ $271{ }^{\circ} \mathrm{C}$ (decomp); IR (neat) $\mathrm{cm}^{-1} 3072,3040,2950,2904,2867,1729,1586$, $1574,1564,1463,1442,1414,1361,1247,1147,1093,1043,992,963,863$, 793, 783, 764, 754, 745, 728, 660; ${ }^{1} \mathrm{H} \mathrm{NMR}\left(400 \mathrm{MHz}, \mathrm{CDCl}_{3}, 300 \mathrm{~K}\right) \delta 9.39$ $9.28(\mathrm{~m}, 1 \mathrm{H}), 8.82(\mathrm{~d}, J=4.1 \mathrm{~Hz}, 1 \mathrm{H}), 8.80-8.73(\mathrm{~m}, 1 \mathrm{H}), 8.51-8.43(\mathrm{~m}, 2 \mathrm{H})$, $8.43(\mathrm{~s}, 1 \mathrm{H}), 8.39(\mathrm{~d}, J=7.6 \mathrm{~Hz}, 1 \mathrm{H}), 8.31(\mathrm{~d}, J=7.9 \mathrm{~Hz}, 1 \mathrm{H}), 7.90(\mathrm{dt}, J=7.7$, $1.7 \mathrm{~Hz}, 1 \mathrm{H}), 7.75-7.63(\mathrm{~m}, 2 \mathrm{H}), 7.60-7.49(\mathrm{~m}, 2 \mathrm{H}) 7.38-7.30(\mathrm{~m}, 1 \mathrm{H}), 1.59$ (s, $9 \mathrm{H}) ;{ }^{13} \mathrm{C} \mathrm{NMR}\left(100 \mathrm{MHz}, \mathrm{CDCl}_{3}, 300 \mathrm{~K}\right) \delta 155.6,148.7,148.0,137.4,137.3,136.7,128.8,128.6$, $128.6,128.5,128.1,127.8,127.0,126.5,125.6,124.8,124.4,124.3,123.8,123.0,122.8,122.8$, 122.2, 121.9, 118.2, 118.0, 35.5, 31.8(3C); HRMS (ESI) $m / z$ calcd for $\mathrm{C}_{30} \mathrm{H}_{24} \mathrm{~N}_{3}[\mathrm{M}+\mathrm{H}]^{+} 426.1970$, found 426.1967 . 


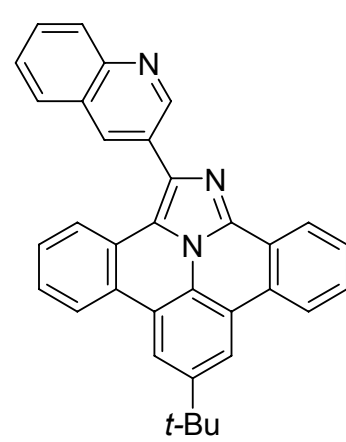

Using procedure A, 3m (26.1 mg, $0.055 \mathrm{mmol}, 55 \%)$ was obtained from $\mathbf{2 m}$ (46 mg, $0.30 \mathrm{mmol}$ ); Pink solid; $R_{\mathrm{f}}: 0.3$ (hexane: ethyl acetate $=8: 2$ ); $\mathrm{mp} 275$ $281{ }^{\circ} \mathrm{C}$ (decomp); IR (neat) $\mathrm{cm}^{-1} 3060,2953,2869,2208,1956,1611,1570$, $1528,1490,1456,1442,1409,1355,1338,1244,1127,1042,944,915,867$, 784, 766, 729, 698, 661; ${ }^{1} \mathrm{H}$ NMR (400 MHz, $\left.\mathrm{CDCl}_{3}, 300 \mathrm{~K}\right) ; \delta 9.42(\mathrm{~s}, 1 \mathrm{H})$, 8.80-8.74 (m, 1H), $8.69(\mathrm{~s}, 1 \mathrm{H}), 8.52-8.47(\mathrm{~m}, 2 \mathrm{H}), 8.43(\mathrm{~s}, 1 \mathrm{H}), 8.39(\mathrm{~d}, J=$ $8.0 \mathrm{~Hz}, 1 \mathrm{H}), 8.24(\mathrm{~d}, J=8.8 \mathrm{~Hz}, 1 \mathrm{H}), 8.06(\mathrm{~d}, J=7.6 \mathrm{~Hz}, 1 \mathrm{H}), 7.95(\mathrm{~d}, J=8.0$ $\mathrm{Hz}, 1 \mathrm{H}), 7.80(\mathrm{t}, J=7.2 \mathrm{~Hz}, 1 \mathrm{H}), 7.74-7.65(\mathrm{~m}, 2 \mathrm{H}), 7.63(\mathrm{t}, J=7.6 \mathrm{~Hz}, 1 \mathrm{H})$, $7.51(\mathrm{t}, J=7.6 \mathrm{~Hz}, 1 \mathrm{H}), 7.34(\mathrm{t}, J=7.6 \mathrm{~Hz}, 1 \mathrm{H}), 1.62(\mathrm{~s}, 9 \mathrm{H}) ;{ }^{13} \mathrm{C}$ NMR $(100$ $\left.\mathrm{MHz}, \mathrm{CDCl}_{3}, 300 \mathrm{~K}\right) \delta 151.8,148.2,147.6,138.0,137.1,136.6,134.5,129.7,129.4,128.8,128.8$, $128.7,128.2,128.1,128.1,127.7,127.0,126.8,126.3,125.2,124.2,123.6,123.5,123.2,122.9$, 122.7, 121.9, 120.7, 118.4, 118.1, 35.6, 31.9 (3C); HRMS (ESI) $m / z$ calcd for $\mathrm{C}_{34} \mathrm{H}_{26} \mathrm{~N}_{3}[\mathrm{M}+\mathrm{H}]^{+}$ 476.2127, found 476.2123.

\section{8-tert-butyl-2-(thiophen-2-yl)tribenzo[b,g,ij]imidazo[2,1,5-de]quinolizine (3n):}

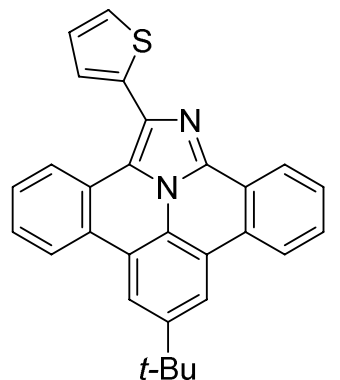

Using procedure A, 3n (15.5 mg, $0.036 \mathrm{mmol}, 36 \%)$ was obtained from $2 \mathbf{n}$ (33 $\mathrm{mg}, 0.30 \mathrm{mmol}$ ); Pink solid; $R_{\mathrm{f}}: 0.3$ (hexane:ethyl acetate $=17: 3$ ); Yellow solid; mp 310-318 ${ }^{\circ} \mathrm{C}$ (decomp); IR (neat) $\mathrm{cm}^{-1} 3057,2952,2905,2867,1613$, $1574,1530,1479,1443,1414,1381,1361,1269,1230,1201,1122,1043$, $1029,951,930,865,848,836,783,761,728,696 ;{ }^{1} \mathrm{H} \mathrm{NMR}\left(400 \mathrm{MHz}, \mathrm{CDCl}_{3}\right.$, $300 \mathrm{~K}) \delta 8.78-8.72(\mathrm{~m}, 1 \mathrm{H}), 8.51-8.43(\mathrm{~m}, 2 \mathrm{H}), 8.41(\mathrm{~s}, 1 \mathrm{H}), 8.37(\mathrm{~d}, J=8.4$ $\mathrm{Hz}, 1 \mathrm{H}), 8.34(\mathrm{~d}, J=8.0,1 \mathrm{H}), 7.72-7.62(\mathrm{~m}, 2 \mathrm{H}), 7.58-7.47(\mathrm{~m}, 3 \mathrm{H}), 7.42(\mathrm{t}, J$ $=7.6 \mathrm{~Hz}, 1 \mathrm{H}), 7.24(\mathrm{dd}, J=5.2,0.9 \mathrm{~Hz}, 1 \mathrm{H}), 1.58(\mathrm{~s}, 9 \mathrm{H}) ;{ }^{13} \mathrm{C} \mathrm{NMR}\left(100 \mathrm{MHz}, \mathrm{CDCl}_{3}, 300 \mathrm{~K}\right) \delta$ $148.0,138.0,137.3,130.9,128.7,128.6,128.6,128.0,127.7,127.4,127.3,126.8,126.8,126.3$, 125.4, 124.4, 123.7, 123.6, 123.2, 122.8, 122.0, 121.9, 120.6, 118.3, 118.0, 35.5, 31.8 (3C); HRMS (ESI) $m / z$ calcd for $\mathrm{C}_{29} \mathrm{H}_{23} \mathrm{~N}_{2} \mathrm{~S}[\mathrm{M}+\mathrm{H}]^{+} 431.1582$, found 431.1579 .

\section{2-benzhydryl-8-tert-butyltribenzo[b,g,ij] imidazo[2,1,5-de]quinolizine (30):}

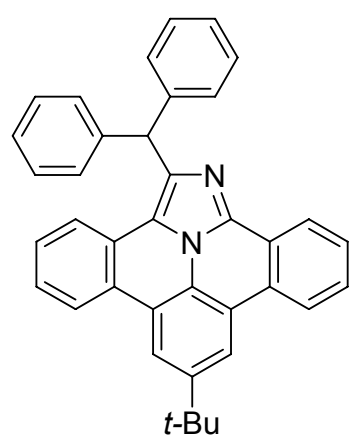

Using procedure A, $3 \mathbf{0}$ (41 mg, $0.080 \mathrm{mmol}, 80 \%)$ was obtained from 20 (193 $\mathrm{mg}, 1.0 \mathrm{mmol}$ ); Yellow solid; $R_{\mathrm{f}}: 0.3$ (hexane:dichloromethane $=6: 4$ ); $\mathrm{mp}$ 300-303 ${ }^{\circ} \mathrm{C}$ (decomp); IR (neat) $\mathrm{cm}^{-1} 2960,2924,2852,1724,1661,1611$, $1570,1523,1491,1449,1440,1412,1386,1365,1259,1217,1076,1026$, $862,801,782,761,737,712,666 ;{ }^{1} \mathrm{H}$ NMR (400 MHz, $\left.\mathrm{CDCl}_{3}, 300 \mathrm{~K}\right) \delta$ 8.66-8.62 (m, 1H), 8.48-8.35 (m, 4H), 8.15-8.09 (m, 1H), 7.67-7.58 (m, 2H), $7.50-7.40(\mathrm{~m}, 6 \mathrm{H}), 7.31(\mathrm{t}, J=7.6 \mathrm{~Hz}, 4 \mathrm{H}), 7.21(\mathrm{t}, J=7.6 \mathrm{~Hz}, 2 \mathrm{H}), 6.34(\mathrm{~s}$, $1 \mathrm{H}), 1.57(\mathrm{~s}, 9 \mathrm{H}) ;{ }^{13} \mathrm{C} \mathrm{NMR}\left(100 \mathrm{MHz}, \mathrm{CDCl}_{3}, 300 \mathrm{~K}\right) \delta 147.6,143.2(2 \mathrm{C})$, $139.9,136.8,129.7$ (4C), 128.7, 128.5, 128.3 (4C), 128.1, 127.7, 126.7, 126.7, 126.5, 126.4 (2C), 125.9, 124.5, 124.1, 124.0, 123.3, 122.7, 122.6, 121.8, 119.8, 118.1, 117.8, 51.8, 35.5, 31.9 (3C); HRMS (ESI) $m / z$ calcd for $\mathrm{C}_{38} \mathrm{H}_{31} \mathrm{~N}_{2}[\mathrm{M}+\mathrm{H}]^{+}$515.2487, found 515.2482. 


\section{8-tert-butyl-2-cyclohexyltribenzo $[b, g, i j]$ imidazo[2,1,5-de]quinolizine (3p):}

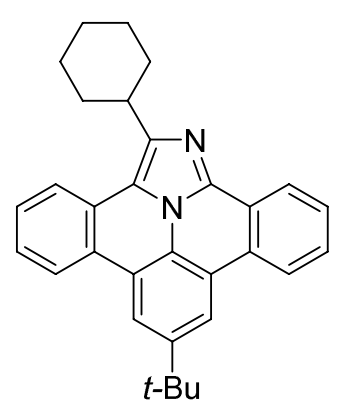

Using procedure A, 3p (7.8 mg, $0.018 \mathrm{mmol}, 18 \%$ ) was obtained from $\mathbf{2 p}$ (33 $\mathrm{mg}, 0.30 \mathrm{mmol}$ ); Yellow solid; $R_{\mathrm{f}}: 0.4$ (hexane:dichloromethane $=6: 4$ ); $\mathrm{mp}$ 220-227 ${ }^{\circ} \mathrm{C}$; IR (neat); $\mathrm{cm}^{-1} 2958,2926,2847,1725,1667,1609,1570,1528$, $1484,1456,1440,1413,1386,1360,1333,1252,1203,1169,1118,1024,987$, 973, 943, 890, 863, 852, 821, 782, 757, 726, 694, 681; ${ }^{1} \mathrm{H}$ NMR (400 MHz, $\left.\mathrm{CDCl}_{3}, 300 \mathrm{~K}\right) \delta 8.73(\mathrm{~d}, J=8.0 \mathrm{~Hz}, 1 \mathrm{H}), 8.48-8.34(\mathrm{~m}, 4 \mathrm{H}), 8.17(\mathrm{~d}, J=8.4$ $\mathrm{Hz}, 1 \mathrm{H}), 7.69-7.56(\mathrm{~m}, 3 \mathrm{H}), 7.50(\mathrm{t}, J=7.6 \mathrm{~Hz}, 1 \mathrm{H}), 3.44$ (tt, $J=11.6,3.2 \mathrm{~Hz}$, $1 \mathrm{H}), 2.19(\mathrm{~d}, J=11.4 \mathrm{~Hz}, 2 \mathrm{H}), 2.12-1.94(\mathrm{~m}, 4 \mathrm{H}), 1.86(\mathrm{~d}, J=12.0 \mathrm{~Hz}, 1 \mathrm{H})$, $1.57(\mathrm{~s}, 9 \mathrm{H}), 1.54-1.42(\mathrm{~m}, 3 \mathrm{H}) ;{ }^{13} \mathrm{C}$ NMR $\left(100 \mathrm{MHz}, \mathrm{CDCl}_{3}, 300 \mathrm{~K}\right) \delta 147.4,144.5,136.4,128.9$, $128.4,127.9,127.6,126.8,126.6,126.5,126.1,124.2,123.9,123.7,123.3,122.7,121.8,118.1$, 117.9, 117.7, 117.6, 39.0, 35.5, 32.4 (2C), 31.9 (3C), 27.0 (2C), 26.2; HRMS (ESI) $\mathrm{m} / z$ calcd for $\mathrm{C}_{31} \mathrm{H}_{31} \mathrm{~N}_{2}[\mathrm{M}+\mathrm{H}]^{+}$431.2487, found 431.2493.

\section{8-tert-butyl-2-(2,4,6-trimethylbenzyl)tribenzo[b,g,ij]imidazo[2,1,5-de]quinolizine (3q):}

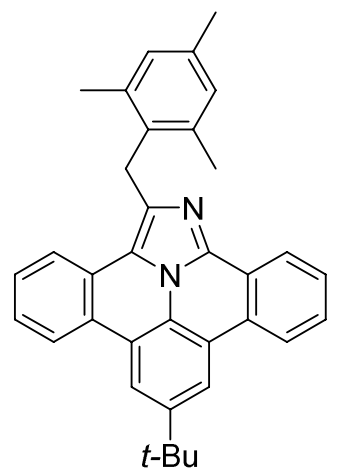

Using procedure A, 3q (20.5 mg, $0.043 \mathrm{mmol}, 43 \%$ ) was obtained from $\mathbf{2 q}$ (48 $\mathrm{mg}, 0.30 \mathrm{mmol}$ ); Yellow solid; $R_{\mathrm{f}}: 0.4$ (hexane:dichloromethane $=1: 1$ ); $\mathrm{mp}$ 272-276 ${ }^{\circ} \mathrm{C}$; IR (neat) $\mathrm{cm}^{-1} 2949,2860,1727,1648,1612,1573,1478,1456$, 1440, 1413, 1390, 1362, 1338, 1275, 1249, 1196, 1149, 1121, 1026, 1012, 998, 896, 886, 866, 850, 782, 750, 729, 697, 678; ${ }^{1} \mathrm{H}$ NMR (400 MHz, $\mathrm{CDCl}_{3}, 300$ K) $\delta 8.59-8.54(\mathrm{~m}, 1 \mathrm{H}), 8.47-8.37(\mathrm{~m}, 4 \mathrm{H}), 8.33(\mathrm{~d}, J=7.2 \mathrm{~Hz}, 1 \mathrm{H}), 7.66-7.49$ $(\mathrm{m}, 4 \mathrm{H}), 6.94(\mathrm{~s}, 2 \mathrm{H}), 4.63(\mathrm{~s}, 2 \mathrm{H}), 2.39-2.32(\mathrm{~m}, 9 \mathrm{H}), 1.57(\mathrm{~s}, 9 \mathrm{H}) ;{ }^{13} \mathrm{C} \mathrm{NMR}$ $\left(100 \mathrm{MHz}, \mathrm{CDCl}_{3}, 300 \mathrm{~K}\right) \delta 147.5,137.5,137.1,136.6(2 \mathrm{C}), 135.6,133.8$, $128.8,128.6,128.4,128.1,128.0$ (2C), 127.5, 126.7, 126.6, 126.3, 124.5, 123.9, 123.4, 123.0, 122.7, 122.6, 121.8, 119.0, 118.0, 117.7, 35.5, 31.9 (3C), 29.7 (2C), 29.3, 22.7; HRMS (ESI) $m / z$ calcd for $\mathrm{C}_{35} \mathrm{H}_{33} \mathrm{~N}_{2}[\mathrm{M}+\mathrm{H}]^{+} 481.2644$, found 481.2643 . 


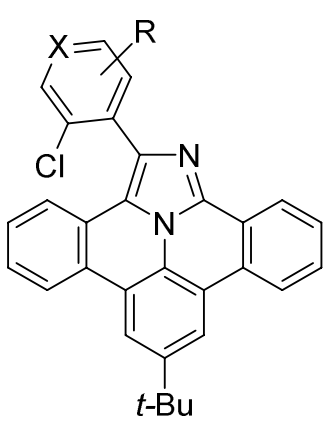

3

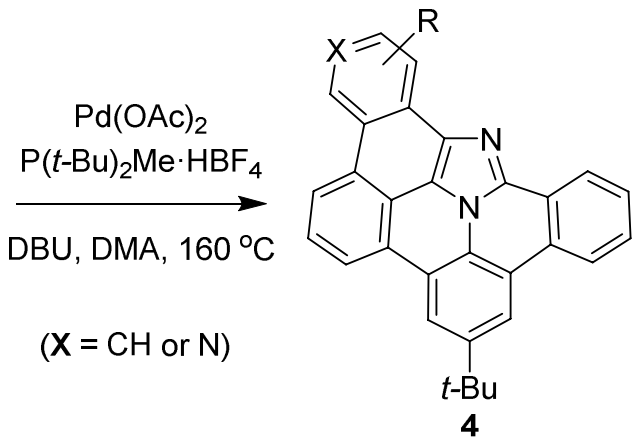

4

To a mixture of 3 (1.0 equiv), palladium(II) acetate ( 0.60 equiv), di-t-butylmethylphosphonium tetrafluoroborate (1.8 equiv) was added a degassed mixture of DMA $(2.0 \mathrm{~mL})$ and DBU $(0.50 \mathrm{~mL})$, and the mixture was stirred for $5 \mathrm{~min}$ at room temperature. Then the reaction solution was stirred for 9-20 $\mathrm{h}$ at $160{ }^{\circ} \mathrm{C}$. After cooled to room temperature and diluted with toluene, the mixture was washed with water four times, brine one time, dried with sodium sulfate, and filtered with Celite. After evaporation, the crude mixture was purified by silica gel column chromatography (hexane/ethyl acetate $=2 / 1$ ) to yield the title compound 4 .

\section{9-tert-butyl-14b ${ }^{1}, 15$-diazadibenzo $[f g, i j]$ cyclopenta $[r s t]$ pentaphene (4a):}

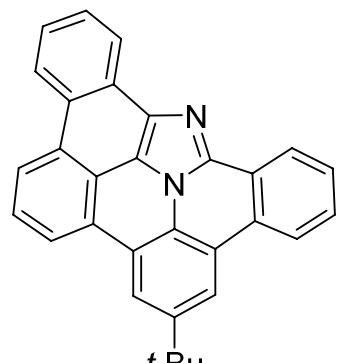

$t$-Bu

4a (10.8 mg, $0.026 \mathrm{mmol}, 43 \%$ ) was obtained from 3a (27.5 mg, $0.060 \mathrm{mmol})$; 20 h; Yellow solid; $R_{\mathrm{f}}: 0.3$ (hexane:ethyl acetate $=4: 1$ ); $\Phi 0.38 ; \mathrm{mp} \mathrm{282-}$ $286^{\circ} \mathrm{C}$ (decomp); IR (neat); $\mathrm{cm}^{-1} 3046,2952,2921,2853,1730,1669,1616$, $1597,15773,1542,1525,1447,1439,1381,1360,1328,1254,1202,1147$, $1120,1091,1042,1017,963,896,866,813,777,746,732,693 ;{ }^{1} \mathrm{H}$ NMR (400 MHz, $\left.\mathrm{CD}_{2} \mathrm{Cl}_{2} / \mathrm{CS}_{2}, 300 \mathrm{~K}\right) \delta 8.79-8.74(\mathrm{~m}, 1 \mathrm{H}), 8.66(\mathrm{~d}, J=8.4 \mathrm{~Hz}, 2 \mathrm{H})$, $8.56(\mathrm{~d}, J=8.4 \mathrm{~Hz}, 1 \mathrm{H}), 8.55-8.49(\mathrm{~m}, 1 \mathrm{H}), 8.47(\mathrm{~d}, J=2.8 \mathrm{~Hz}, 2 \mathrm{H}), 8.32(\mathrm{~d}$, $J=8.0 \mathrm{~Hz}, 1 \mathrm{H}), 7.81(\mathrm{t}, J=7.6 \mathrm{~Hz}, 1 \mathrm{H}), 7.78-7.69(\mathrm{~m}, 3 \mathrm{H}), 7.61(\mathrm{t}, J=7.8 \mathrm{~Hz}, 1 \mathrm{H}), 1.64(\mathrm{~s}, 9 \mathrm{H})$; ${ }^{13} \mathrm{C}$ NMR $\left(100 \mathrm{MHz}, \mathrm{CD}_{2} \mathrm{Cl}_{2} / \mathrm{CS}_{2}, 300 \mathrm{~K}\right) \delta 148.9,139.2,132.9,130.0,129.6,129.2,129.2,128.9$, $128.7,128.0,126.8,126.7,126.2,125.5,125.0,124.7,124.2,123.7,123.2,122.8,122.3,121.5$, $121.4,120.2,120.1,119.4,119.1,36.0,32.1$ (3C); HRMS (ESI) $m / z$ calcd for $\mathrm{C}_{31} \mathrm{H}_{23} \mathrm{~N}_{2}[\mathrm{M}+\mathrm{H}]^{+}$ 423.1861, found 423.1856 .

\section{9-tert-butyl-3-fluoro-14b ${ }^{1}$,15-diazadibenzo[fg,ij] cyclopenta[rst $]$ pentaphene (4i):}

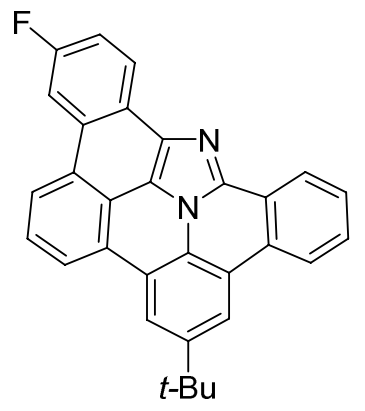

4i (18 mg, $0.041 \mathrm{mmol}, 41 \%$ ) was obtained from 3i (50 mg, $0.10 \mathrm{mmol})$; $9 \mathrm{~h}$; Yellow solid; $R_{\mathrm{f}}$ : 0.2 (hexane:ethyl acetate $=4: 1$ ); $\Phi 0.49 ; \mathrm{mp} 254-258{ }^{\circ} \mathrm{C}$ (decomp); IR (neat); $\mathrm{cm}^{-1} 3067,2950,2905,2868,2216,1671,1601,1576$, $1549,1527,1478,1450,1417,1393,1374,1324,1240,1199,1181,1146$, $1122,1043,969,941,876,851,821,800,759,694,656 ;{ }^{1} \mathrm{H}$ NMR $(400 \mathrm{MHz}$, $\left.\mathrm{CDCl}_{3}, 300 \mathrm{~K}\right) \delta 8.78-8.71(\mathrm{~m}, 1 \mathrm{H}), 8.62(\mathrm{dd}, J=8.4,6.0 \mathrm{~Hz}, 1 \mathrm{H}), 8.51-8.45$ $(\mathrm{m}, 1 \mathrm{H}), 8.43(\mathrm{~s}, 1 \mathrm{H}), 8.40(\mathrm{~s}, 1 \mathrm{H}), 8.34(\mathrm{~d}, J=8.0 \mathrm{~Hz}, 1 \mathrm{H}), 8.25(\mathrm{~d}, J=7.6$ $\mathrm{Hz}, 1 \mathrm{H}), 8.17$ (dd, $J=10.8,2.0 \mathrm{~Hz}, 1 \mathrm{H}), 7.77-7.68(\mathrm{~m}, 3 \mathrm{H}), 7.39$ (dt, $J=8.3$, $1.7 \mathrm{~Hz}, 1 \mathrm{H}), 1.63(\mathrm{~s}, 9 \mathrm{H}) ;{ }^{13} \mathrm{C} \mathrm{NMR}\left(100 \mathrm{MHz}, \mathrm{CDCl}_{3}, 300 \mathrm{~K}\right) \delta 148.4,138.9,132.3,130.6(\mathrm{~d}, J=$ 7.6 Hz), 129.5, 128.9, 128.8, 127.5, 127.5, 126.2, 126.1, 125.8, 124.9, 124.6, 124.5 (d, J=9.0 Hz), $124.2,123.7,123.2,122.4,121.9,120.5,120.1,119.6,119.1,118.6,115.8$ (d, $J=23 \mathrm{~Hz}), 110.1$ (d, $J$ $=23 \mathrm{~Hz}$ ), 35.7, 32.0 (3C); HRMS (ESI) $m / z$ calcd for $\mathrm{C}_{31} \mathrm{H}_{22} \mathrm{FN}_{2}[\mathrm{M}+\mathrm{H}]^{+} 441.1767$, found 441.1761 . 


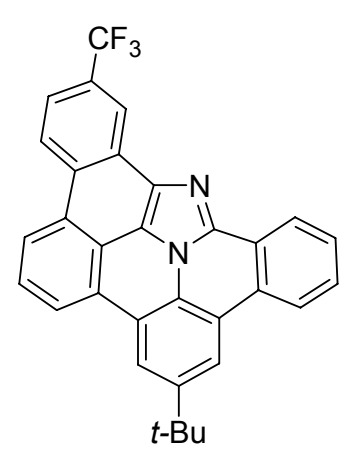

4j (21.4 mg, $0.044 \mathrm{mmol}, 48 \%$ ) was obtained from 3j (46 mg, $0.09 \mathrm{mmol}) ; 19$ h; Yellow green solid; $R_{\mathrm{f}}$ : 0.4 (hexane:ethyl acetate $=10: 1$ ); $\Phi 0.53 ; \mathrm{mp} 339$ $341{ }^{\circ} \mathrm{C}$ (decomp); IR (neat); $\mathrm{cm}^{-1} 3067,2964,2909,2873,2237,1671,1600$, $1572,1523,1471,1457,1428,1394,1360,1319,1299,1270,1243,1214$, $1166,1141,1112,1074,1064,1018,987,954,904,868,845,832,808,780$, 750, 735, 712, 691, 653; ${ }^{1} \mathrm{H}$ NMR $\left(400 \mathrm{MHz}, \mathrm{THF}-d_{8}, 300 \mathrm{~K}\right) \delta 8.77(\mathrm{~s}, 1 \mathrm{H})$, $8.72(\mathrm{~d}, J=8.4 \mathrm{~Hz}, 1 \mathrm{H}), 8.61(\mathrm{~d}, J=8.0 \mathrm{~Hz}, 1 \mathrm{H}), 8.52-8.43(\mathrm{~m}, 3 \mathrm{H}), 8.41(\mathrm{~s}$, $1 \mathrm{H}), 8.19(\mathrm{~d}, J=7.6 \mathrm{~Hz}, 1 \mathrm{H}), 7.72(\mathrm{~d}, J=8.4 \mathrm{~Hz}, 1 \mathrm{H}), 7.68-7.64(\mathrm{~m}, 2 \mathrm{H})$, $7.61(\mathrm{t}, J=8.0 \mathrm{~Hz}, 1 \mathrm{H}), 1.65(\mathrm{~s}, 9 \mathrm{H}) ;{ }^{13} \mathrm{C}$ NMR $\left(100 \mathrm{MHz}, \mathrm{THF}-d_{8}, 300 \mathrm{~K}\right) \delta$ 146.6, 137.1, 129.9, 129.4, 127.7, 127.0, 126.7, 126.6 (q, $J=32 \mathrm{~Hz}), 126.3,125.2,124.6,124.3$, $124.0,123.8,123.6,122.4,122.2,122.0,121.6,121.0,120.8,119.9,118.8,118.5$ (q, $J=2 \mathrm{~Hz}$ ), $117.9,117.7$ (q, $J=3 \mathrm{~Hz}), 117.2,117.0,33.6,29.4$ (3C); HRMS (ESI) $m / z$ calcd for $\mathrm{C}_{32} \mathrm{H}_{22} \mathrm{~F}_{3} \mathrm{~N}_{2}$ $[\mathrm{M}+\mathrm{H}]^{+} 491.1735$ found 491.1733 .

\section{9-tert-butyl-3,14b ${ }^{1}, 15$-triazadibenzo $[f g, i j]$ cyclopenta $[r s t]$ pentaphene (4k):}

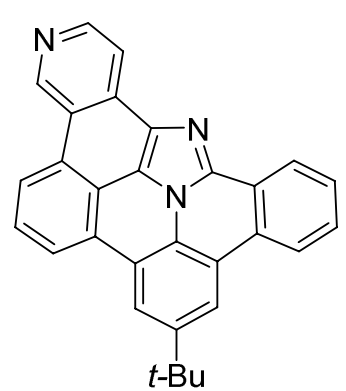

4k (24 mg, $0.057 \mathrm{mmol}, 87 \%$ ) was obtained from 3k (29.8 mg, $0.065 \mathrm{mmol})$; $12 \mathrm{~h}$; Yellow solid; $R_{\mathrm{f}}$ : 0.3 (dichloromethane:methanol $=10: 1$ ); $\Phi 0.46 ; \mathrm{mp}$ 330-332 ${ }^{\circ} \mathrm{C}$ (decomp); IR (neat); $\mathrm{cm}^{-1} 3201,3056,2959,2904,2488,2171$, $1667,1611,1586,1523,1477,1449,1422,1394,1394,1372,1$ 336, 1302, $1287,1244,1214,1171,1147,1122,1056,1017,974,935,870,831,816,767$, 696, 687, 679, 656; ${ }^{1} \mathrm{H}$ NMR (400 MHz, $\left.\mathrm{CDCl}_{3}, 300 \mathrm{~K}\right) \delta 10.0(\mathrm{~s}, 1 \mathrm{H}), 8.95-$ $8.68(\mathrm{~m}, 2 \mathrm{H}), 8.71(\mathrm{~d}, J=8.0 \mathrm{~Hz}, 1 \mathrm{H}), 8.58-8.48(\mathrm{~m}, 4 \mathrm{H}), 8.40(\mathrm{~d}, J=7.6 \mathrm{~Hz}$, $1 \mathrm{H}), 7.93(\mathrm{t}, J=7.8 \mathrm{~Hz}, 1 \mathrm{H}), 7.83-7.75(\mathrm{~m}, 2 \mathrm{H}), 1.67(\mathrm{~s}, 9 \mathrm{H}) ;{ }^{13} \mathrm{C}$ NMR $(100$ $\left.\mathrm{MHz}, \mathrm{CDCl}_{3}, 300 \mathrm{~K}\right) 148.9,140.1,139.7,130.7,129.8,129.5,129.1,127.4,126.2,126.1,126.0$, $124.9,124.1,124.1,123.9,123.2,122.1,121.9,121.8,120.7,120.4,119.9,119.2,119.0,117.1$, 116.4, 35.8, 32.0 (3C); HRMS (ESI) $m / z$ calcd for $\mathrm{C}_{30} \mathrm{H}_{22} \mathrm{~N}_{3}[\mathrm{M}+\mathrm{H}]^{+} 424.1814$, found 424.1818. 


\section{NMR Spectra}
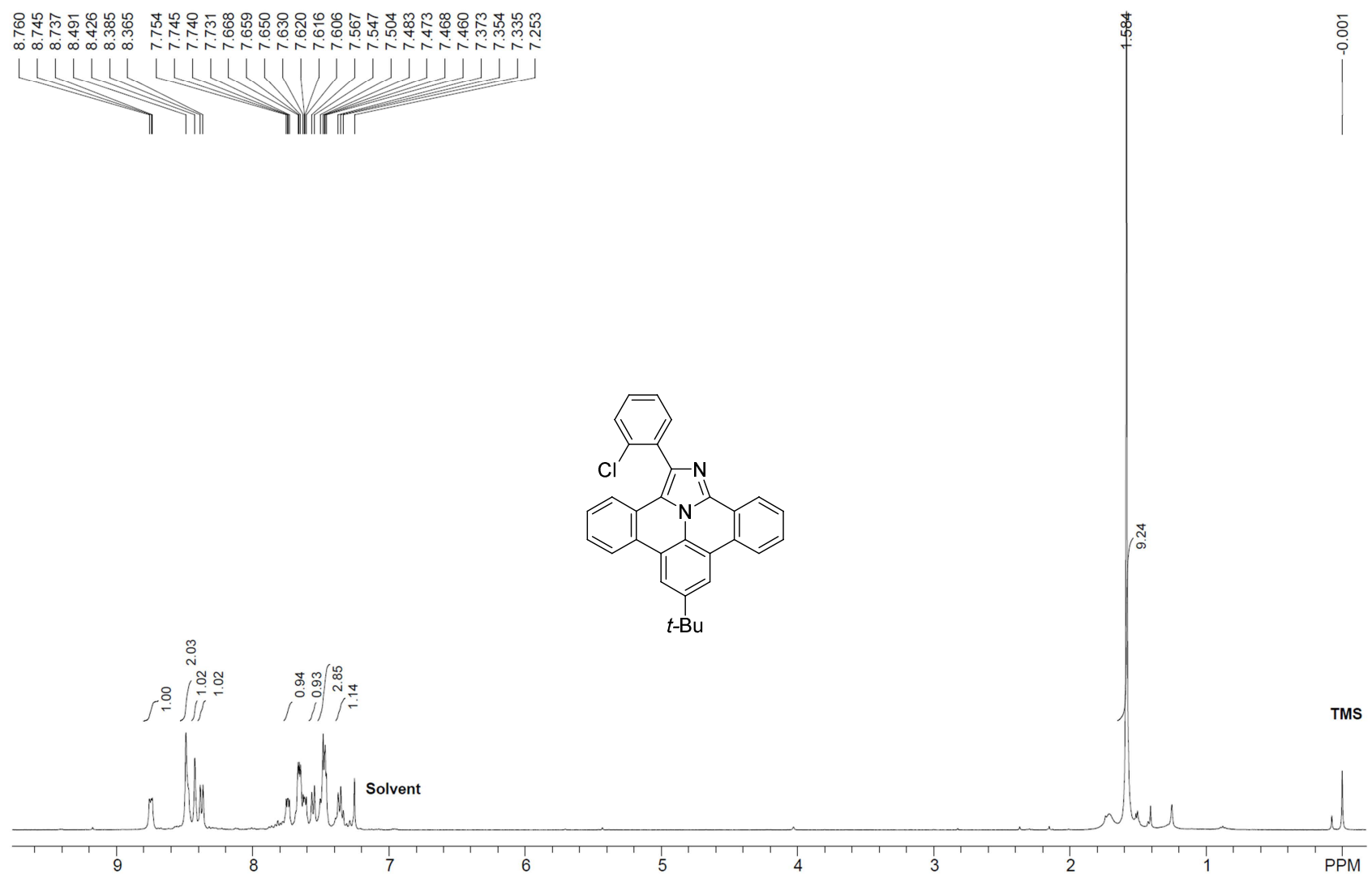

Figure S1. ${ }^{1} \mathrm{H}$ NMR spectrum of $\mathbf{3 a}\left(400 \mathrm{MHz}, \mathrm{CDCl}_{3}, 300 \mathrm{~K}\right)$.
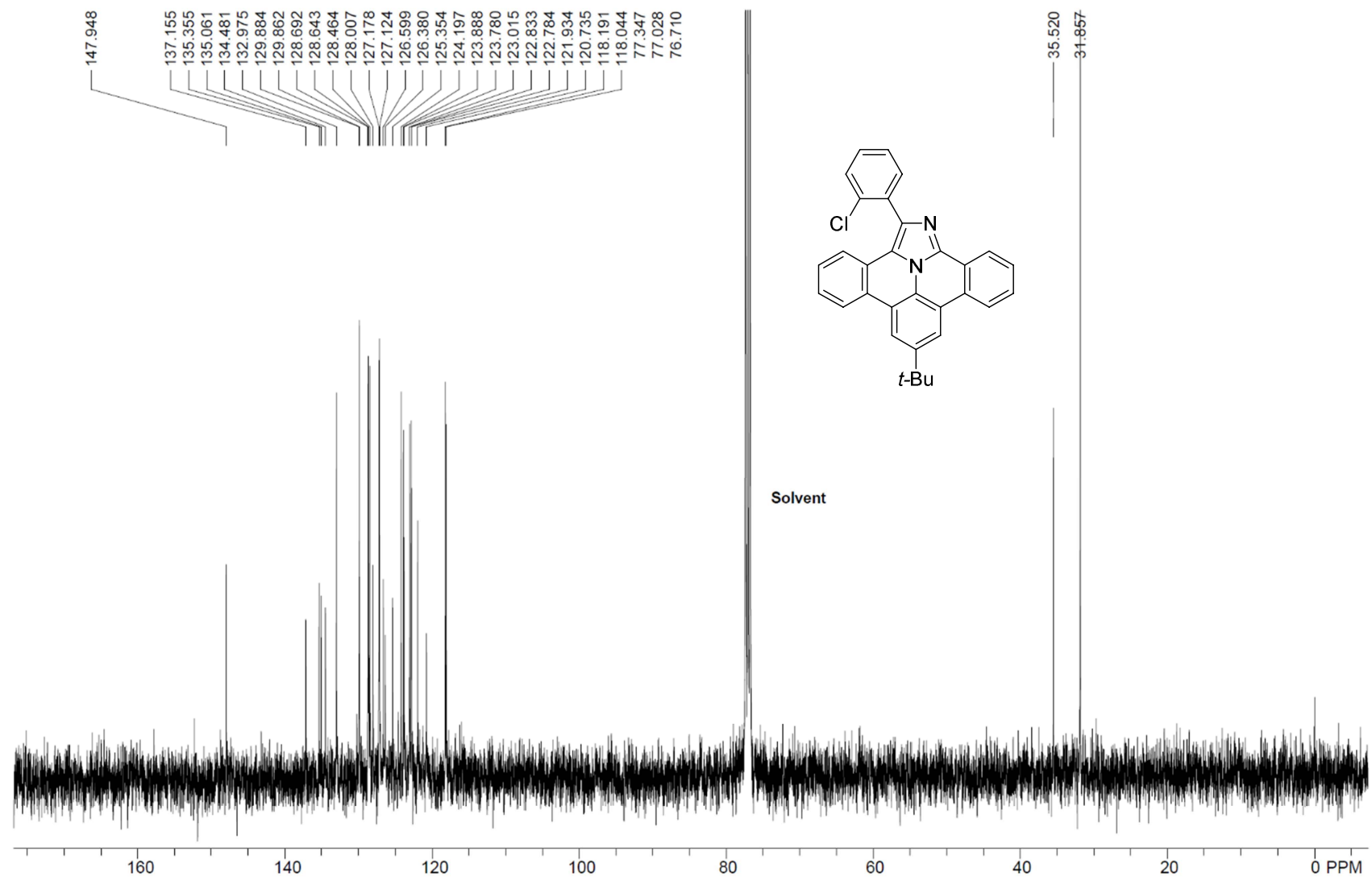

Figure S2. ${ }^{13} \mathrm{C}$ NMR spectrum of $\mathbf{3 a}\left(100 \mathrm{MHz}, \mathrm{CDCl}_{3}, 300 \mathrm{~K}\right)$. 


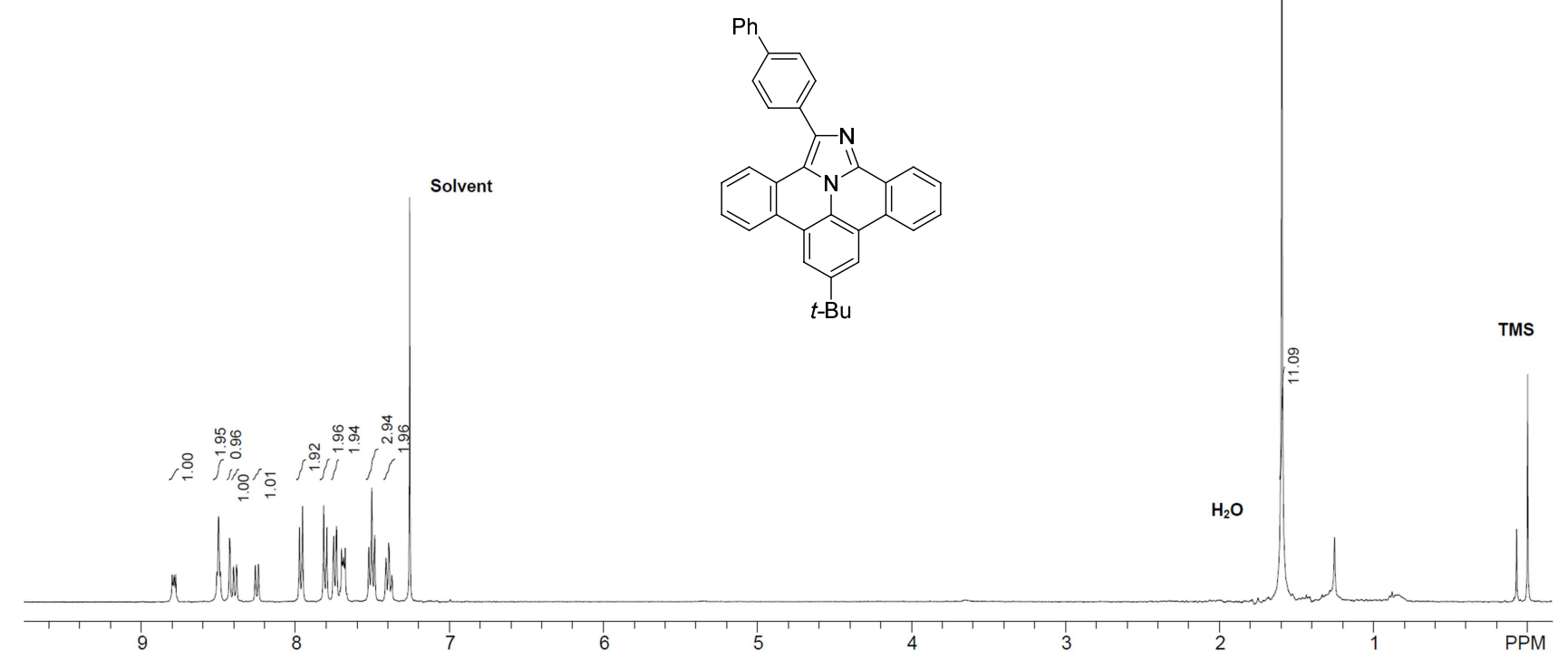

Figure S3. ${ }^{1} \mathrm{H}$ NMR spectrum of $\mathbf{3 b}\left(400 \mathrm{MHz}, \mathrm{CDCl}_{3}, 300 \mathrm{~K}\right)$.

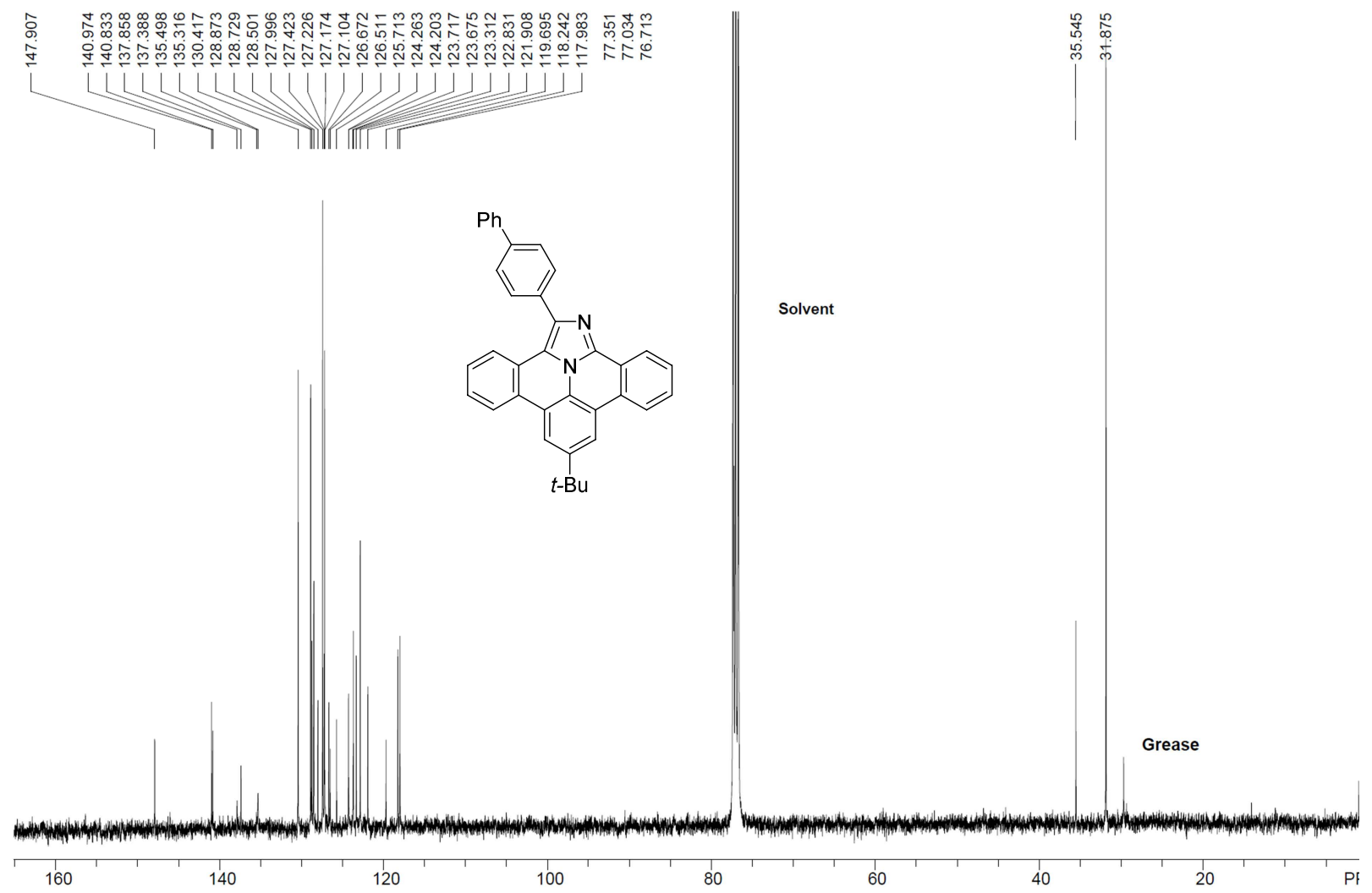

Figure S4. ${ }^{13} \mathrm{C}$ NMR spectrum of $\mathbf{3 b}\left(100 \mathrm{MHz}, \mathrm{CDCl}_{3}, 300 \mathrm{~K}\right)$. 


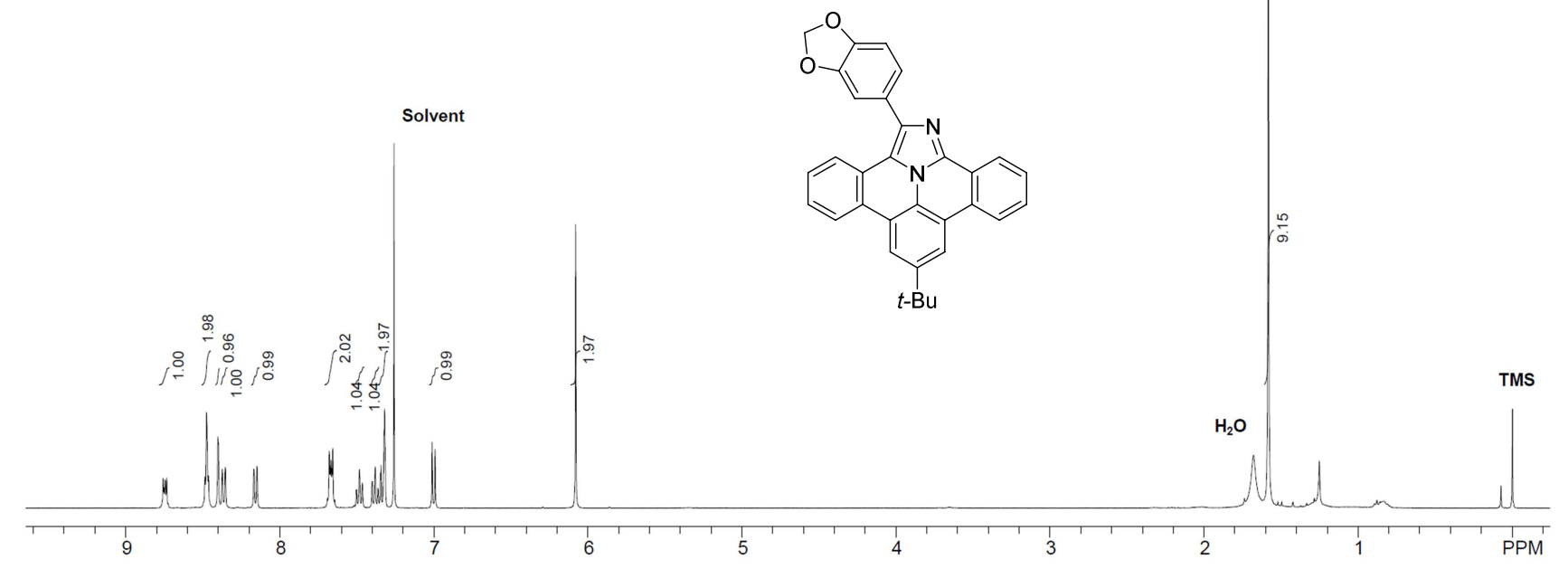

Figure S5. ${ }^{1} \mathrm{H}$ NMR spectrum of $\mathbf{3 c}\left(400 \mathrm{MHz}, \mathrm{CDCl}_{3}, 300 \mathrm{~K}\right)$.

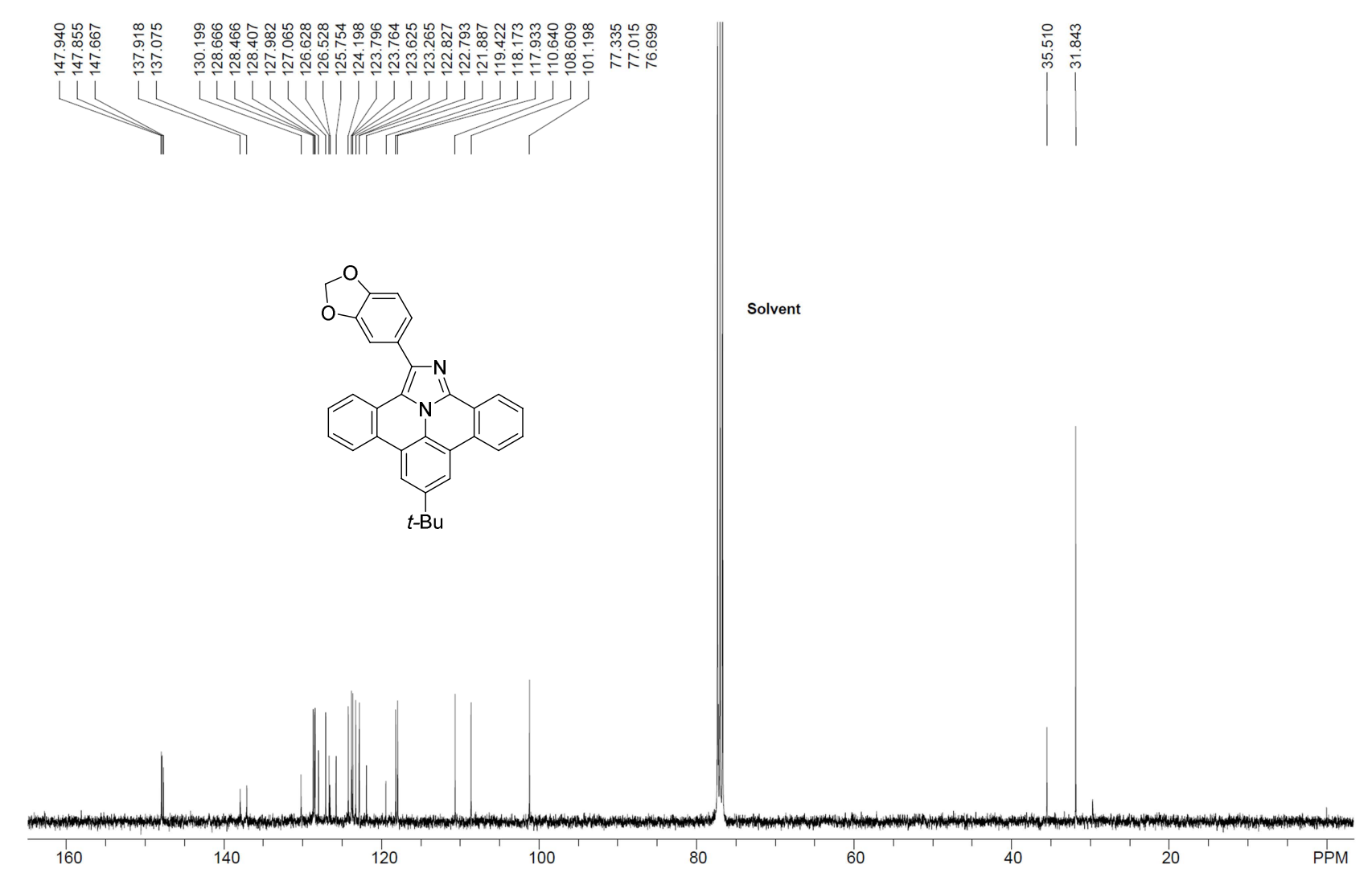

Figure S6. ${ }^{13} \mathrm{C}$ NMR spectrum of $\mathbf{3 c}\left(100 \mathrm{MHz}, \mathrm{CDCl}_{3}, 300 \mathrm{~K}\right)$. 


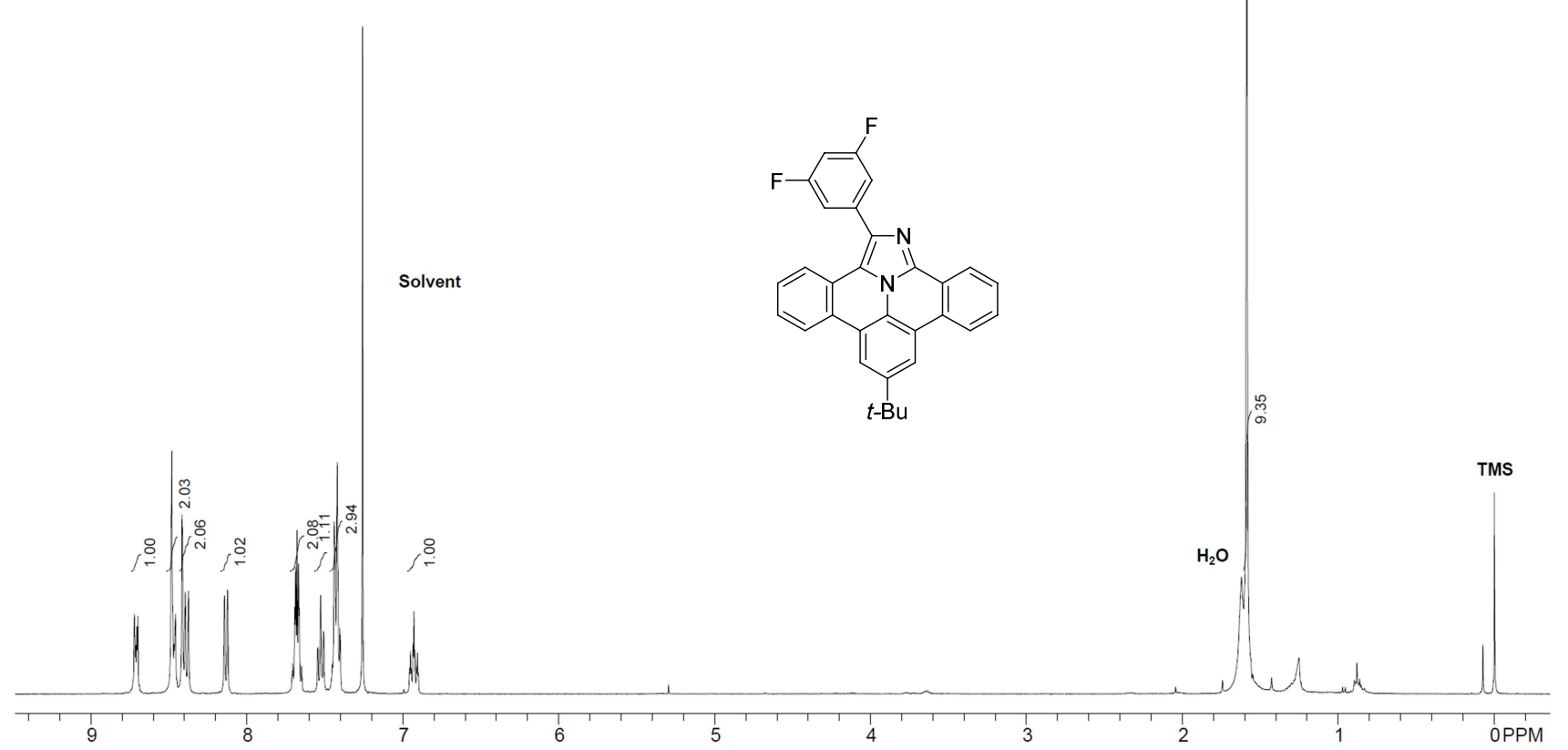

Figure S7. ${ }^{1} \mathrm{H}$ NMR spectrum of $\mathbf{3 d}\left(400 \mathrm{MHz}, \mathrm{CDCl}_{3}, 300 \mathrm{~K}\right)$.

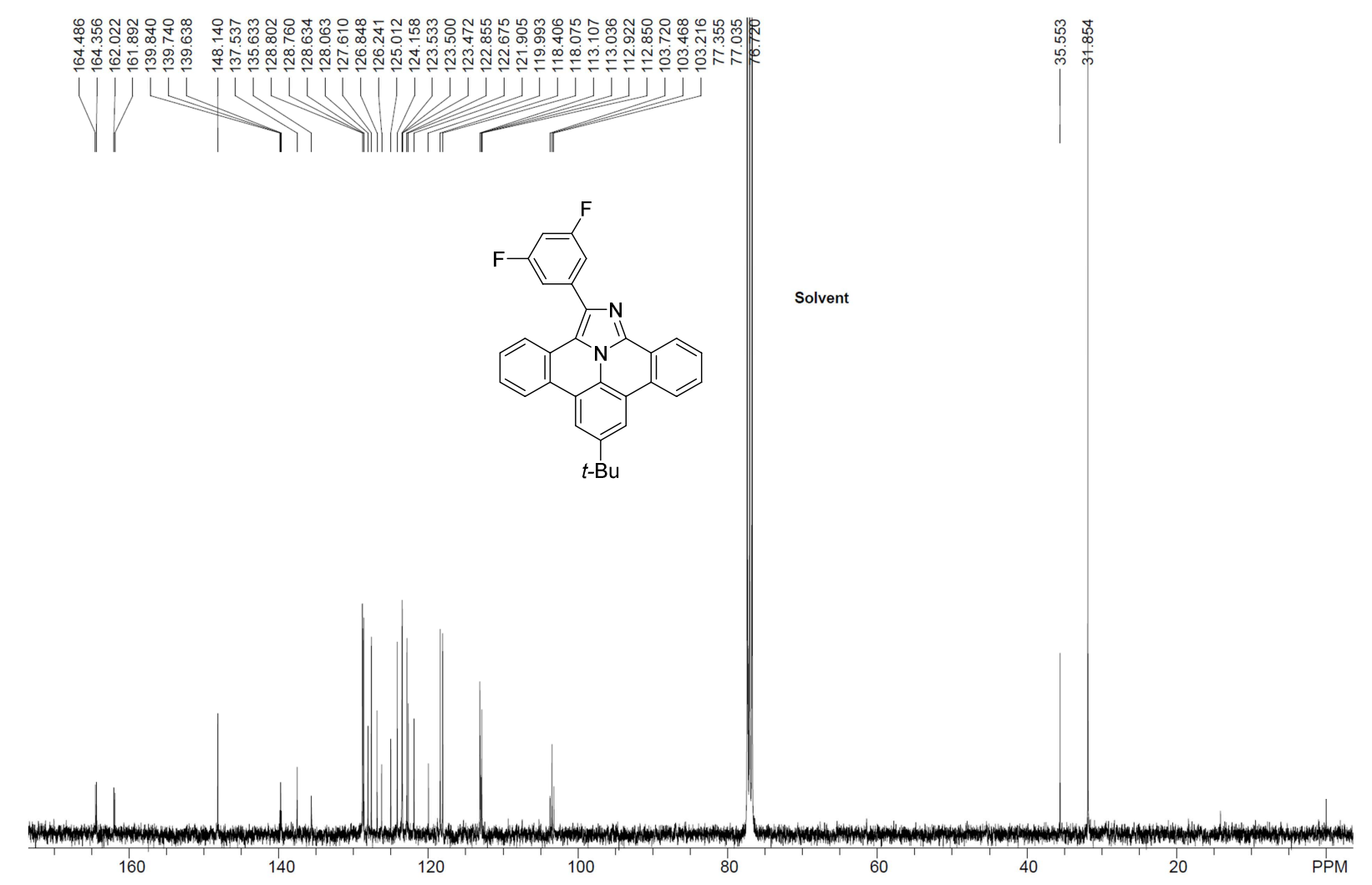

Figure S8. ${ }^{13} \mathrm{C}$ NMR spectrum of $\mathbf{3 d}\left(100 \mathrm{MHz}, \mathrm{CDCl}_{3}, 300 \mathrm{~K}\right)$. 

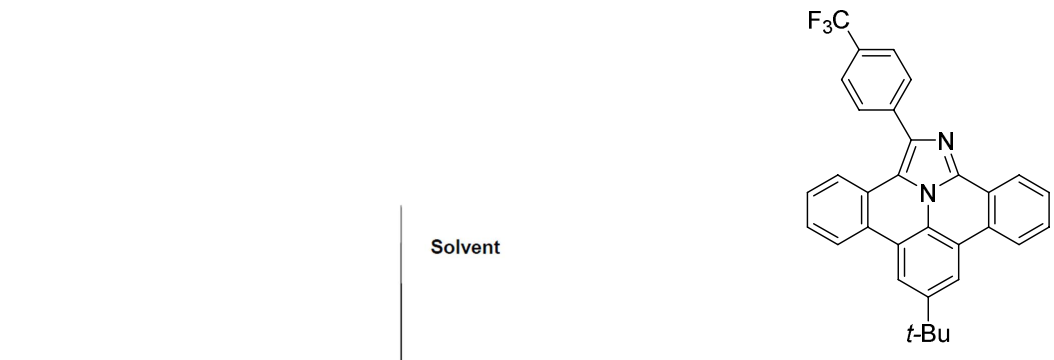

Figure S9. ${ }^{1} \mathrm{H} \mathrm{NMR}$ spectrum of $\mathbf{3 e}\left(400 \mathrm{MHz}, \mathrm{CDCl}_{3}, 300 \mathrm{~K}\right)$.
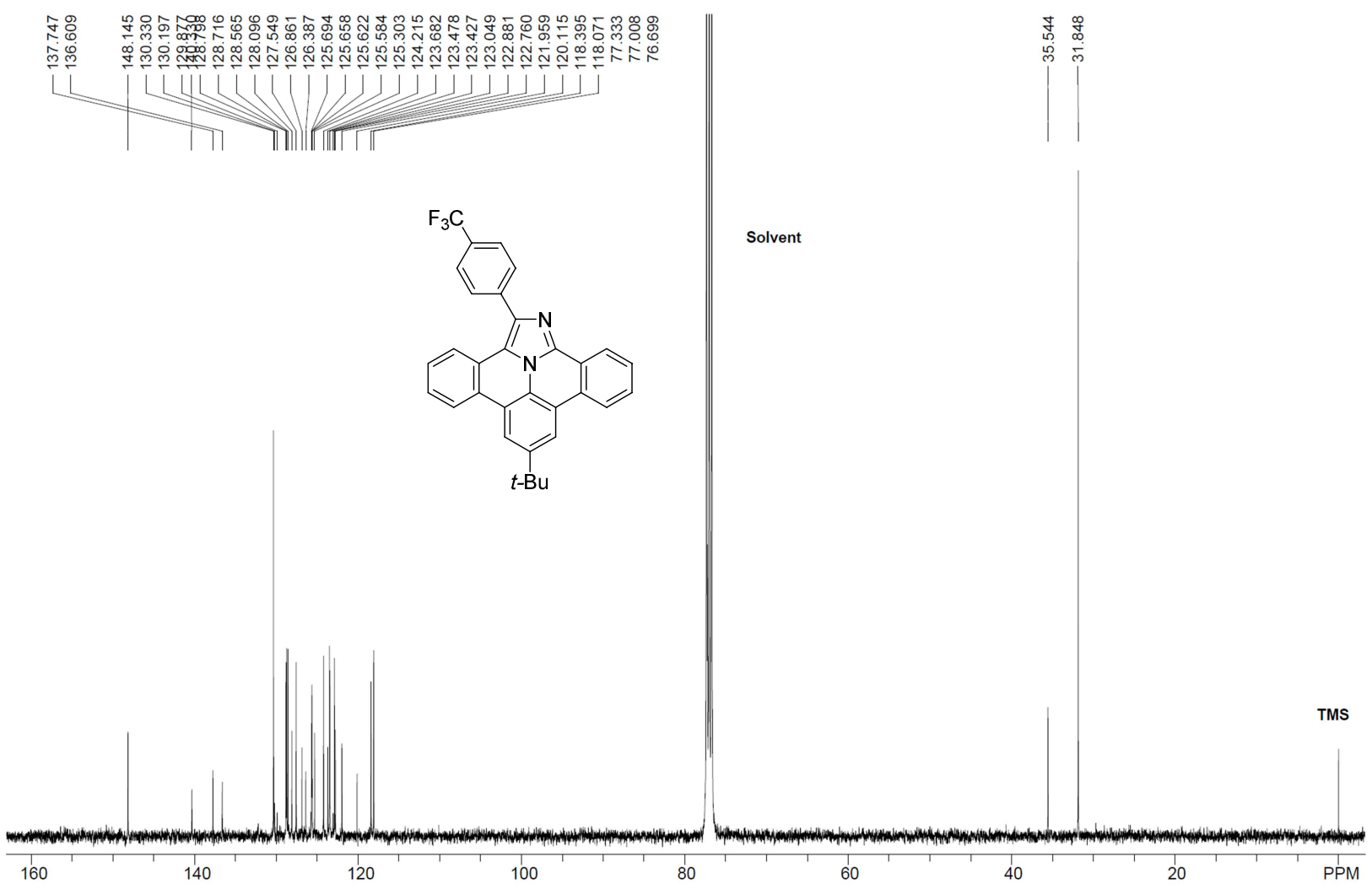

Figure S10. ${ }^{13} \mathrm{C}$ NMR spectrum of $\mathbf{3 e}\left(100 \mathrm{MHz}, \mathrm{CDCl}_{3}, 300 \mathrm{~K}\right)$. 


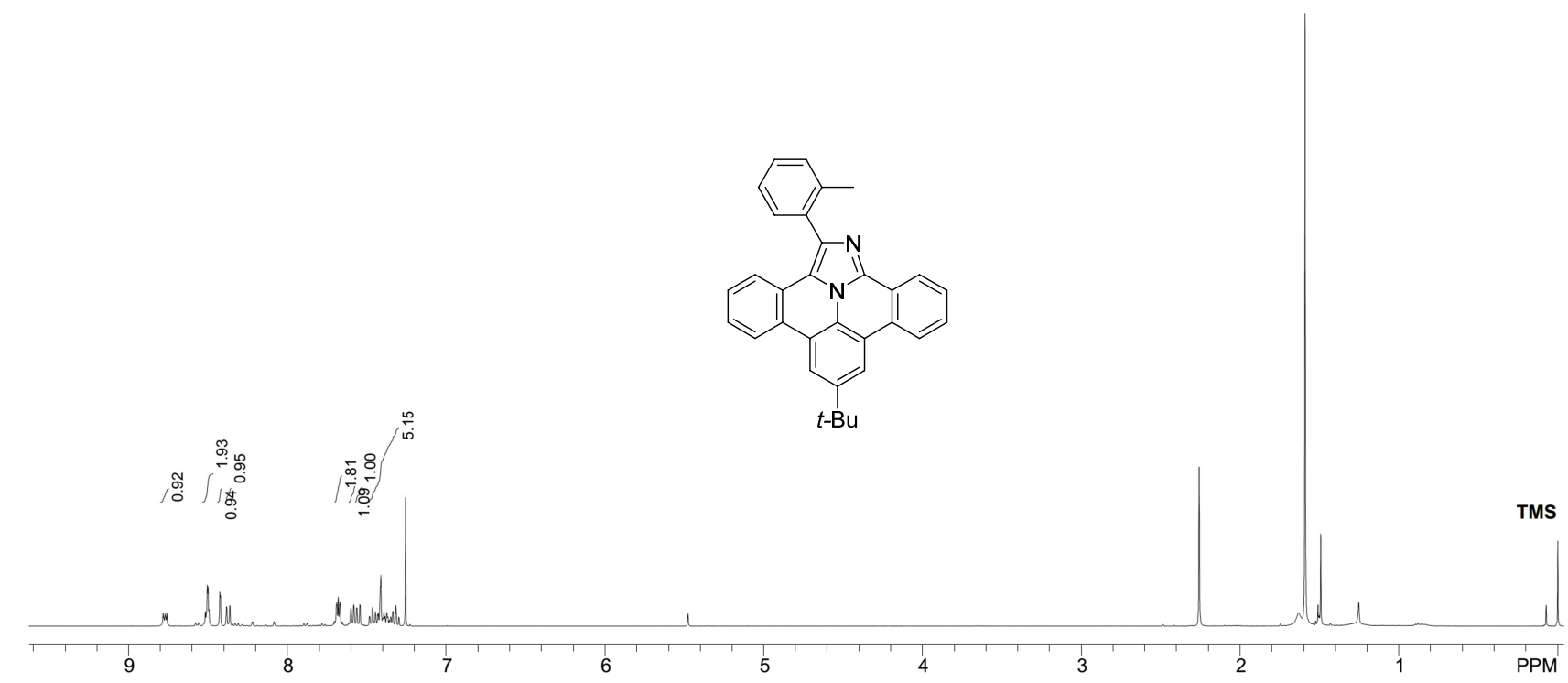

Figure S11. ${ }^{1} \mathrm{H}$ NMR spectrum of $\mathbf{3 f}\left(400 \mathrm{MHz}, \mathrm{CDCl}_{3}, 300 \mathrm{~K}\right)$.
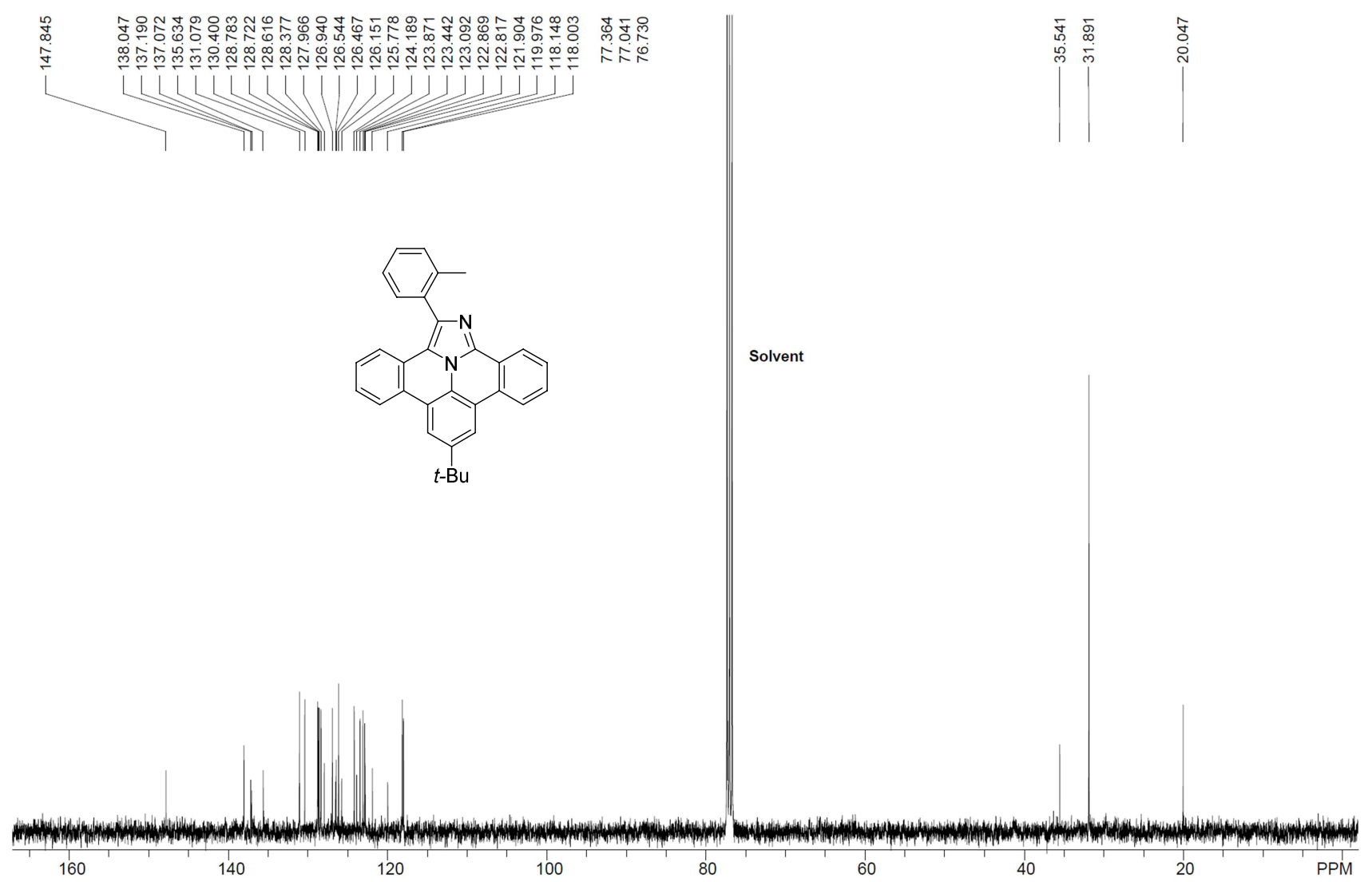

Figure S12. ${ }^{13} \mathrm{C}$ NMR spectrum of $\mathbf{3 f}\left(100 \mathrm{MHz}, \mathrm{CDCl}_{3}, 300 \mathrm{~K}\right)$. 

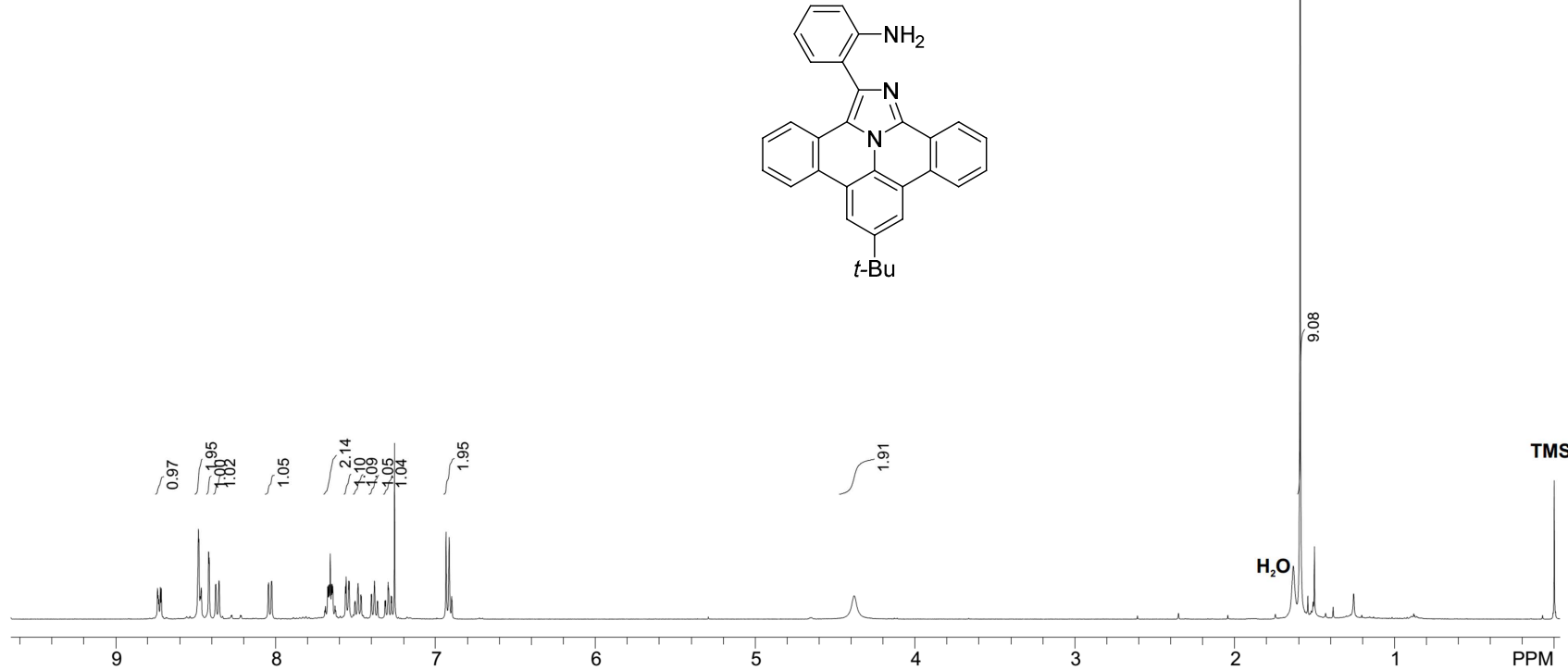

Figure S13. ${ }^{1} \mathrm{H} \mathrm{NMR}$ spectrum of $\mathbf{3 g}\left(400 \mathrm{MHz}, \mathrm{CDCl}_{3}, 300 \mathrm{~K}\right)$.
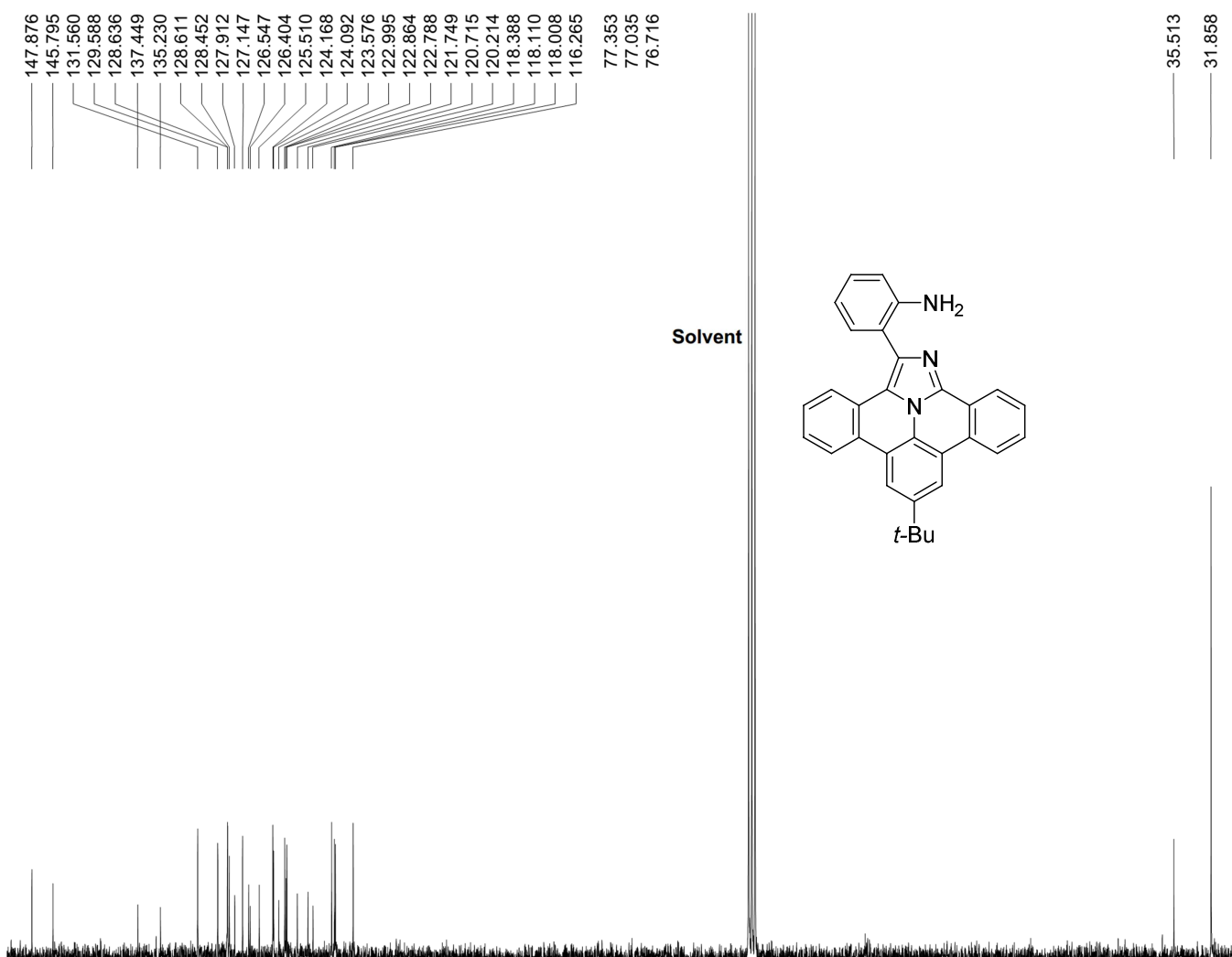

Figure S14. ${ }^{13} \mathrm{C}$ NMR spectrum of $\mathbf{3 g}\left(100 \mathrm{MHz}, \mathrm{CDCl}_{3}, 300 \mathrm{~K}\right)$. 

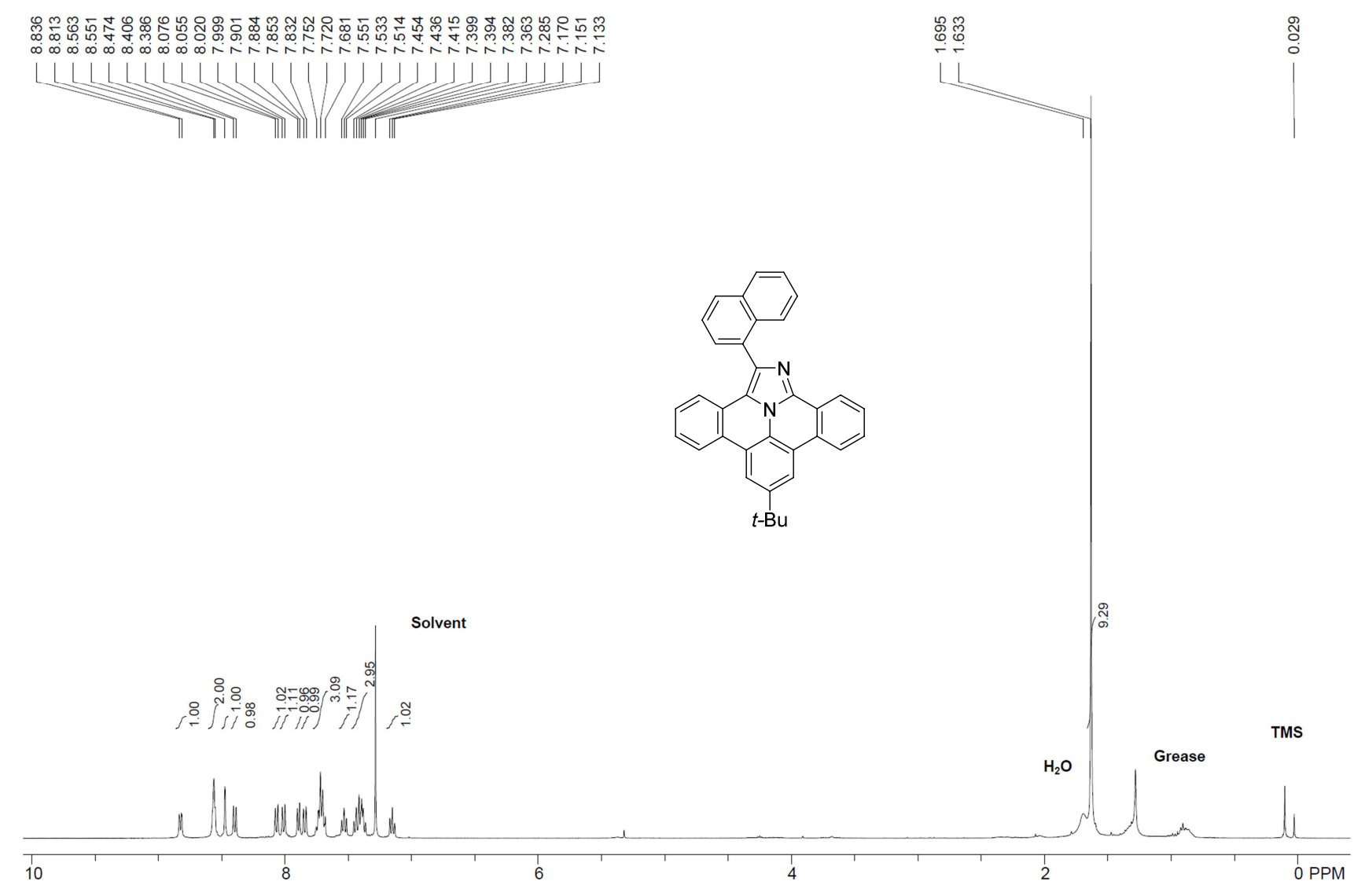

Figure S15. ${ }^{1} \mathrm{H}$ NMR spectrum of $\mathbf{3 h}\left(400 \mathrm{MHz}, \mathrm{CDCl}_{3}, 300 \mathrm{~K}\right)$.

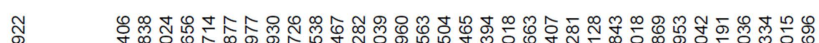

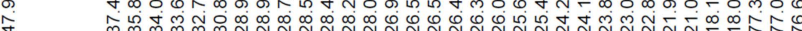

$\left[\quad[]^{2}\right.$

( 2 (

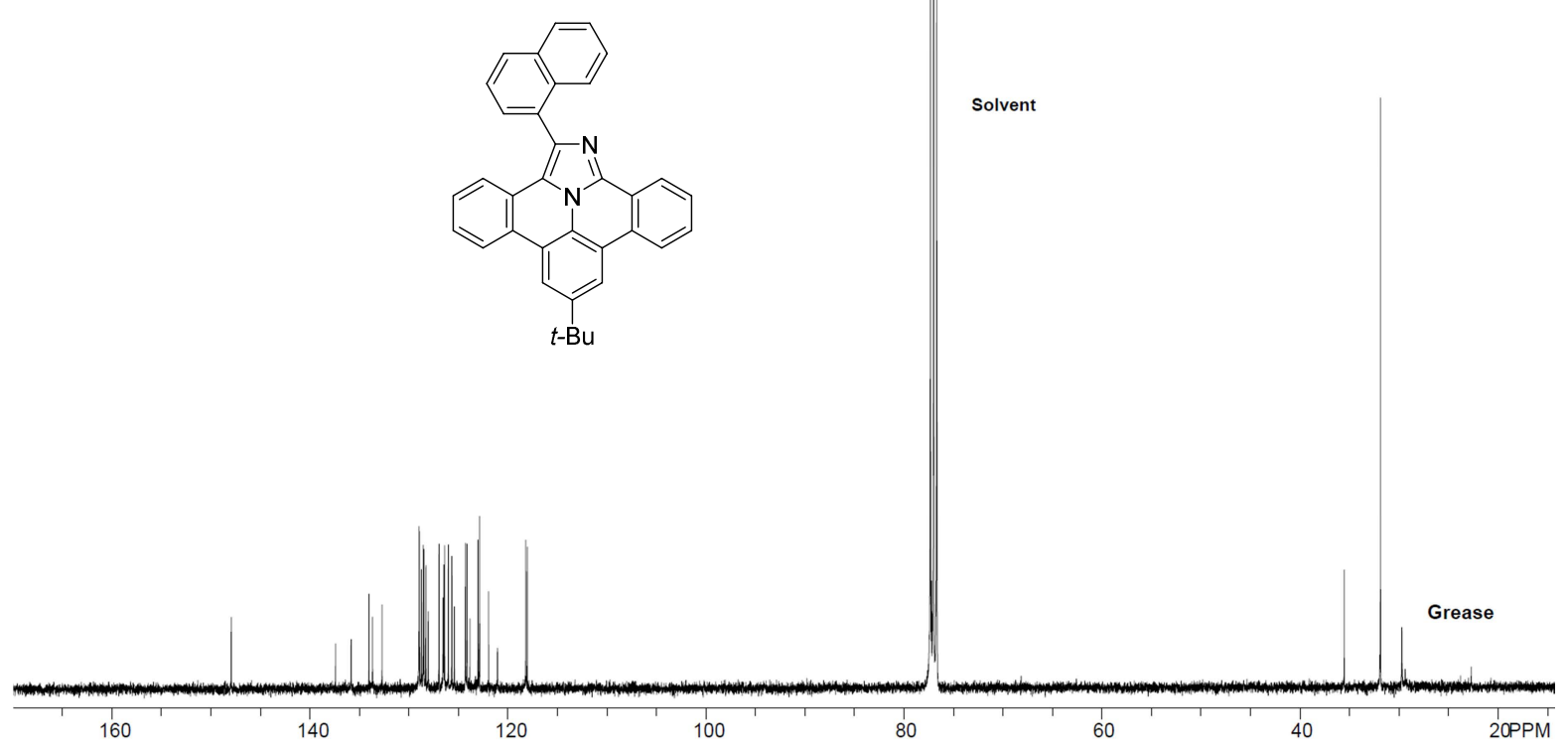

Figure S16. ${ }^{13} \mathrm{C}$ NMR spectrum of $\mathbf{3 h}\left(100 \mathrm{MHz}, \mathrm{CDCl}_{3}, 300 \mathrm{~K}\right)$. 


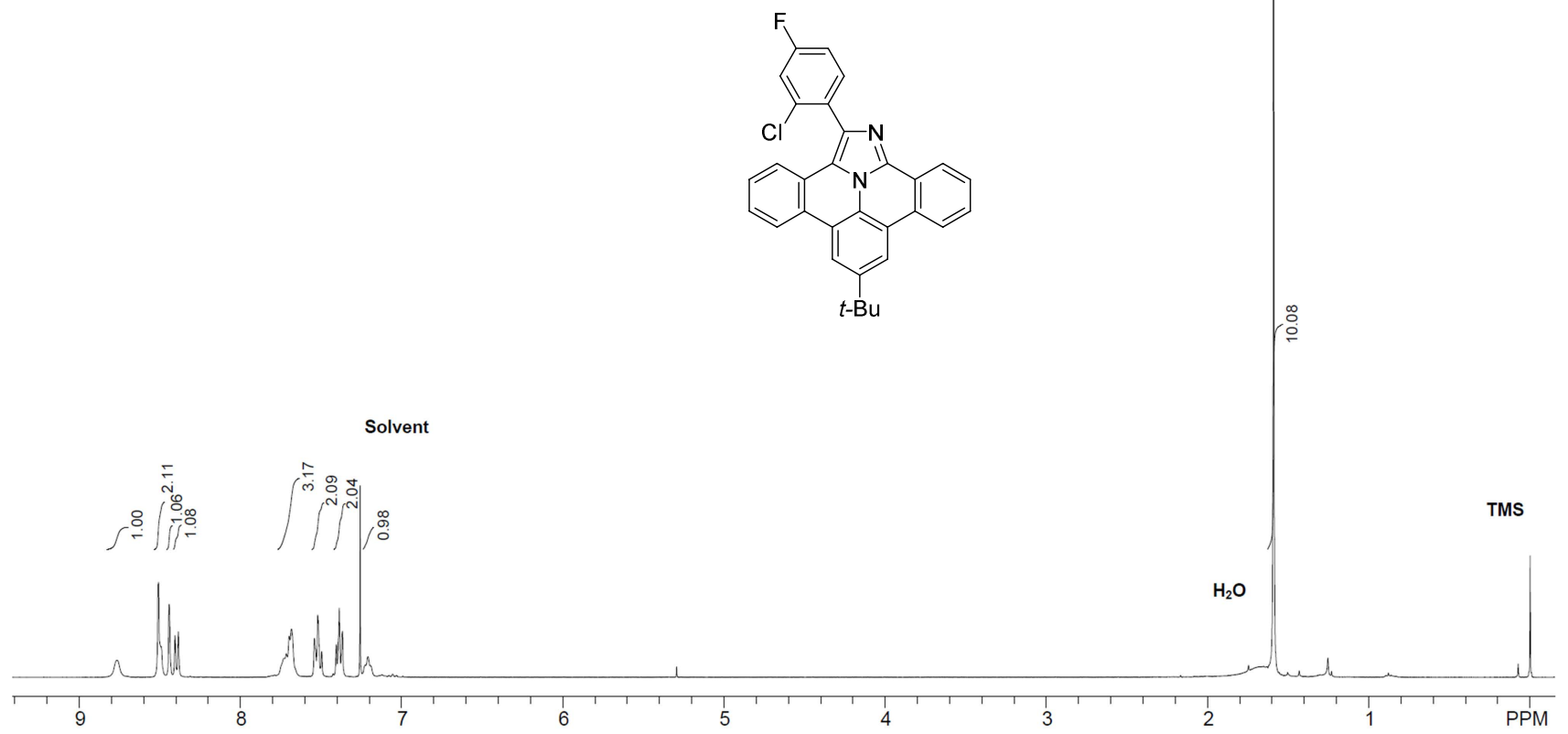

Figure S17. ${ }^{1} \mathrm{H}$ NMR spectrum of $3 \mathbf{i}\left(400 \mathrm{MHz}, \mathrm{CDCl}_{3}, 300 \mathrm{~K}\right)$.
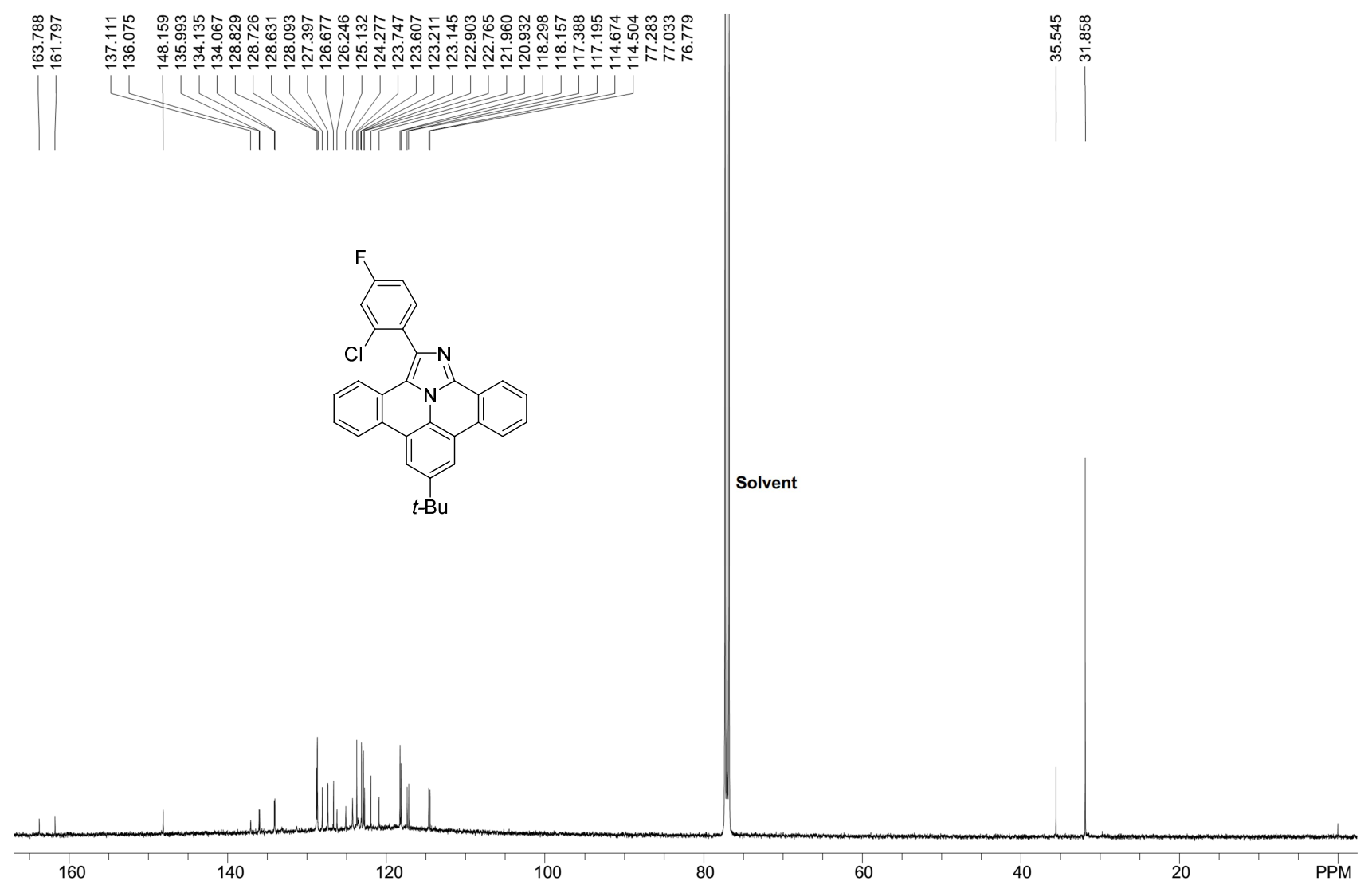

Figure S18. ${ }^{13} \mathrm{C}$ NMR spectrum of $\mathbf{3 i}\left(100 \mathrm{MHz}, \mathrm{CDCl}_{3}, 300 \mathrm{~K}\right)$. 

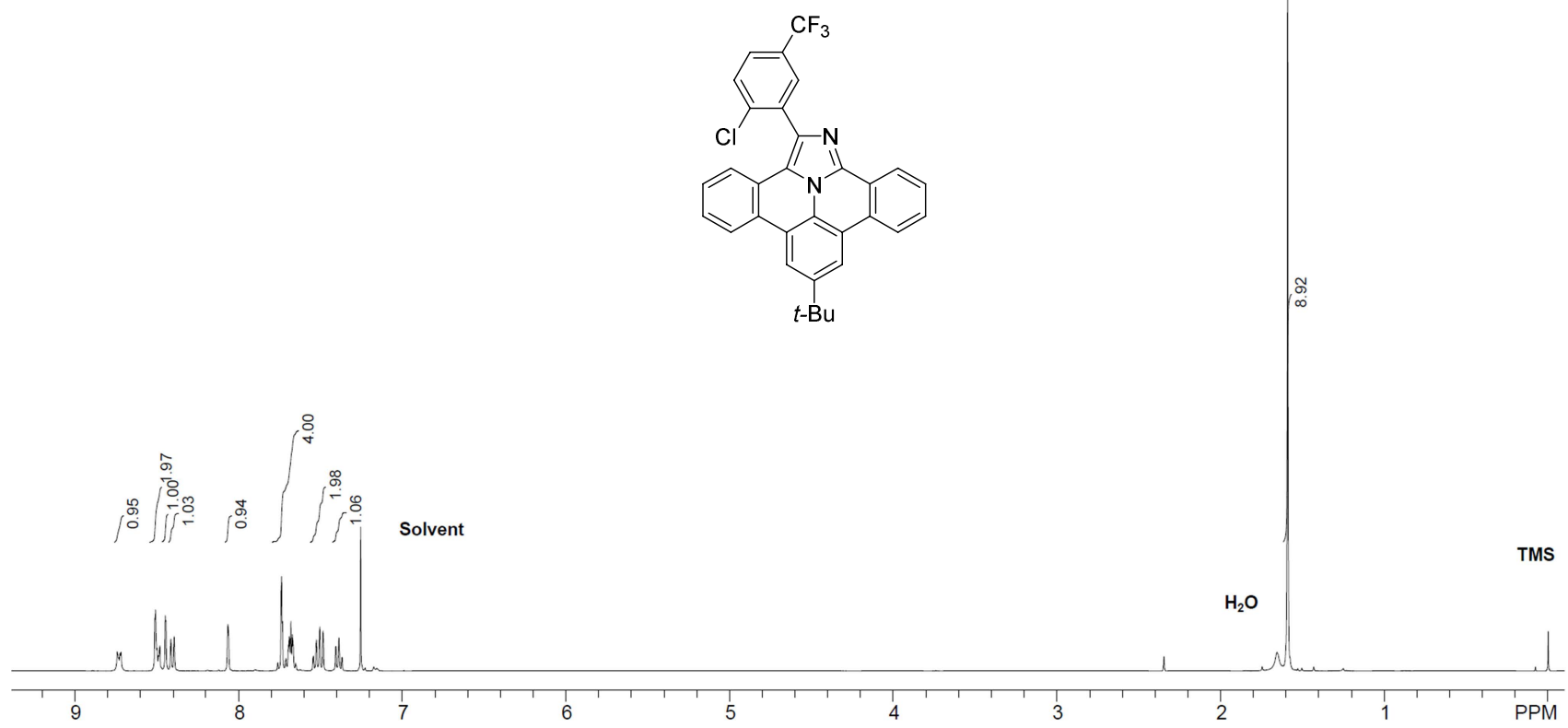

Figure S19. ${ }^{1} \mathrm{H}$ NMR spectrum of $\mathbf{3 j}$ (400 MHz, $\mathrm{CDCl}_{3}, 300 \mathrm{~K}$ ).

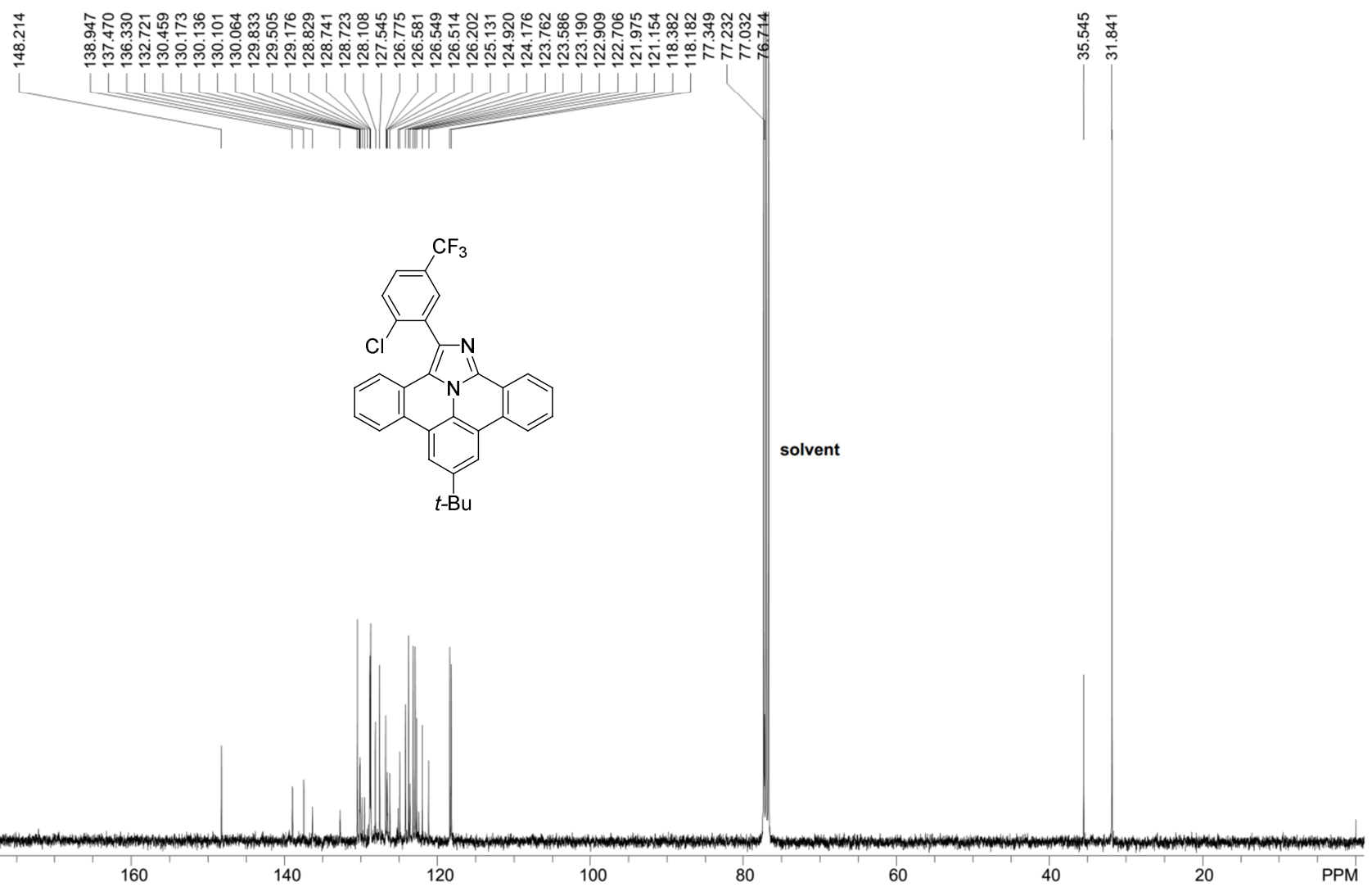

Figure S20. ${ }^{13} \mathrm{C}$ NMR spectrum of $\mathbf{3 j}\left(100 \mathrm{MHz}, \mathrm{CDCl}_{3}, 300 \mathrm{~K}\right)$. 

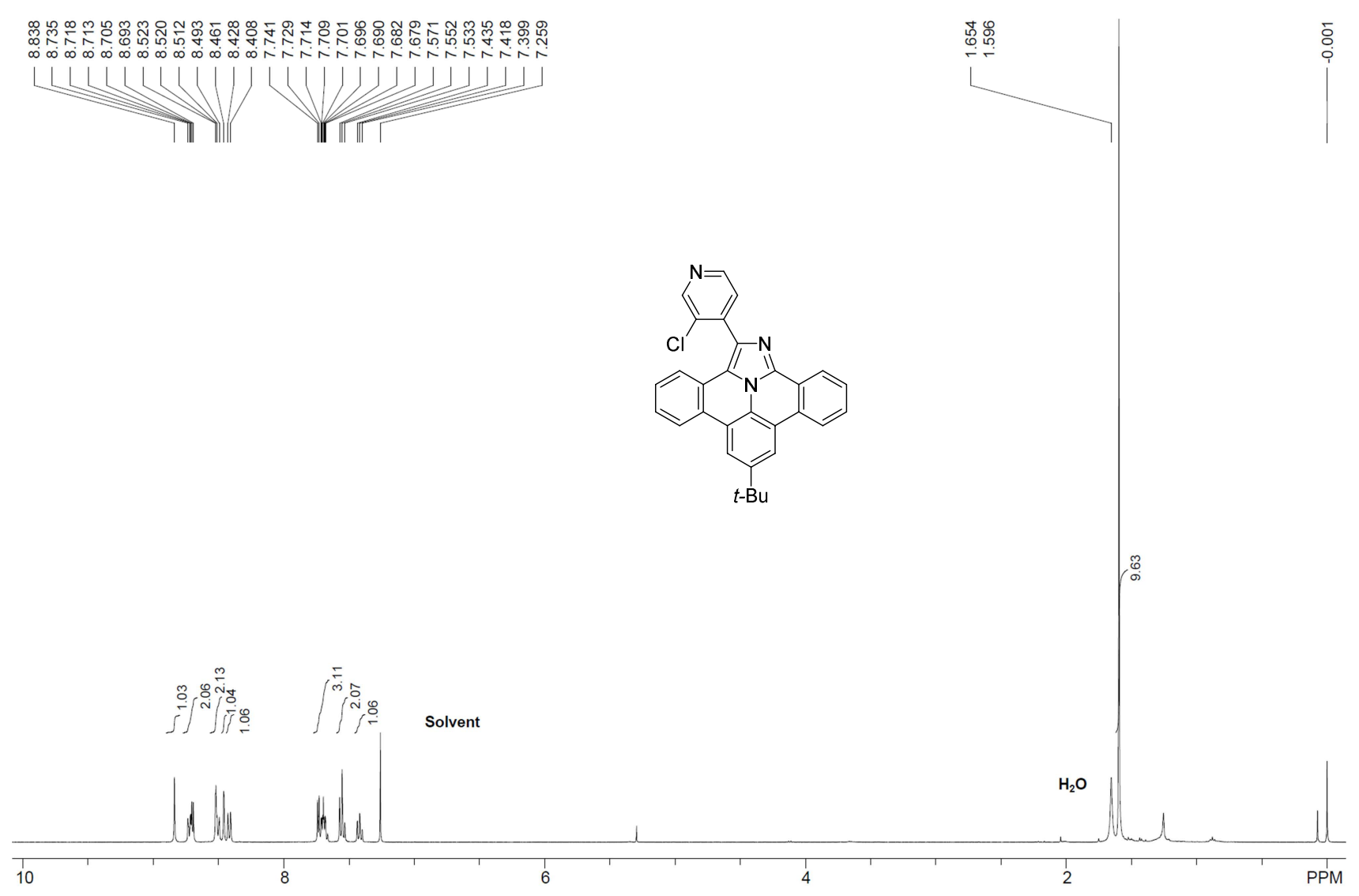

Figure S21. ${ }^{1} \mathrm{H}$ NMR spectrum of $3 \mathbf{k}\left(400 \mathrm{MHz}, \mathrm{CDCl}_{3}, 300 \mathrm{~K}\right)$.
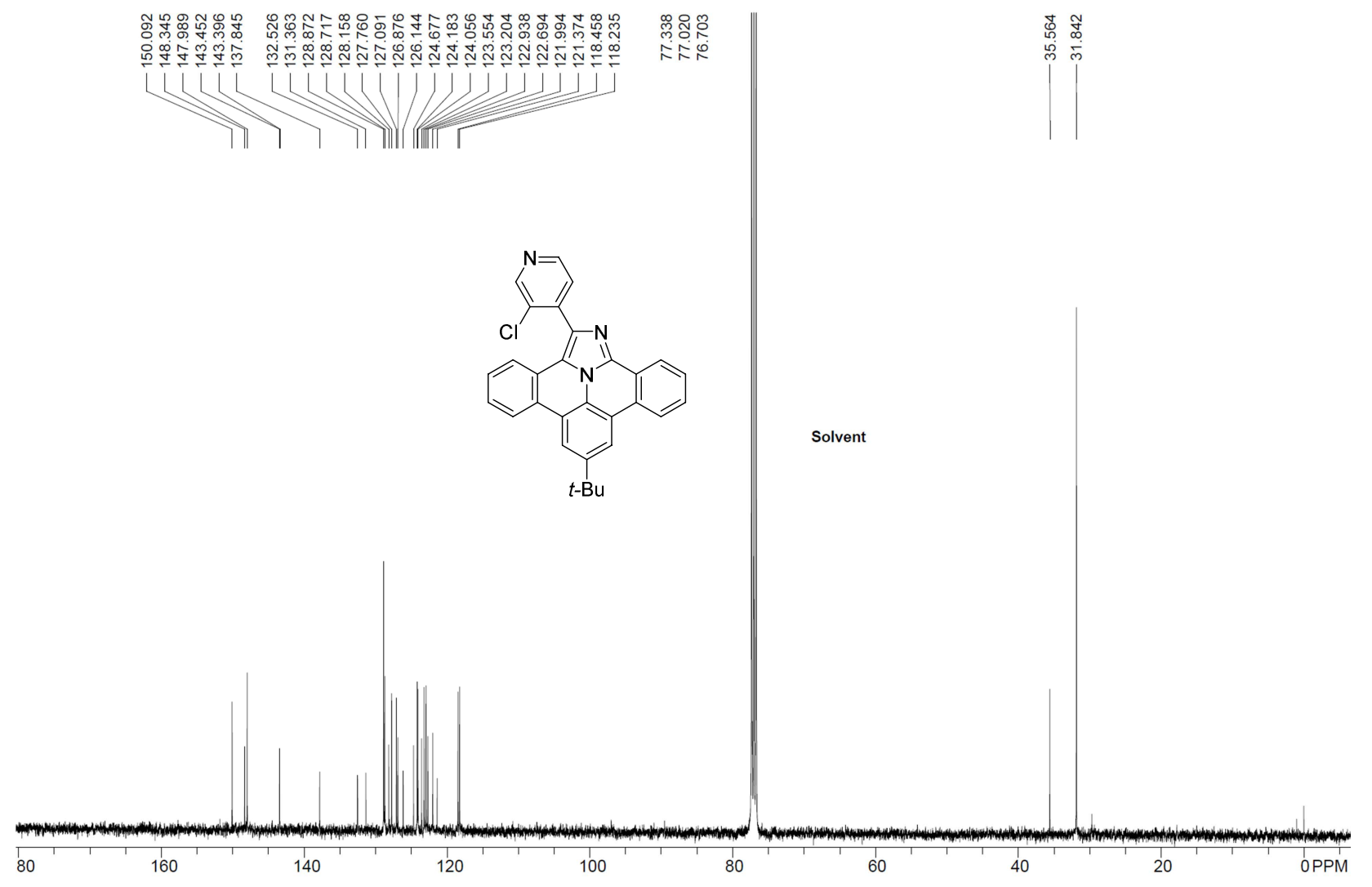

Figure S22. ${ }^{13} \mathrm{C}$ NMR spectrum of $\mathbf{3 k}\left(100 \mathrm{MHz}, \mathrm{CDCl}_{3}, 300 \mathrm{~K}\right)$. 


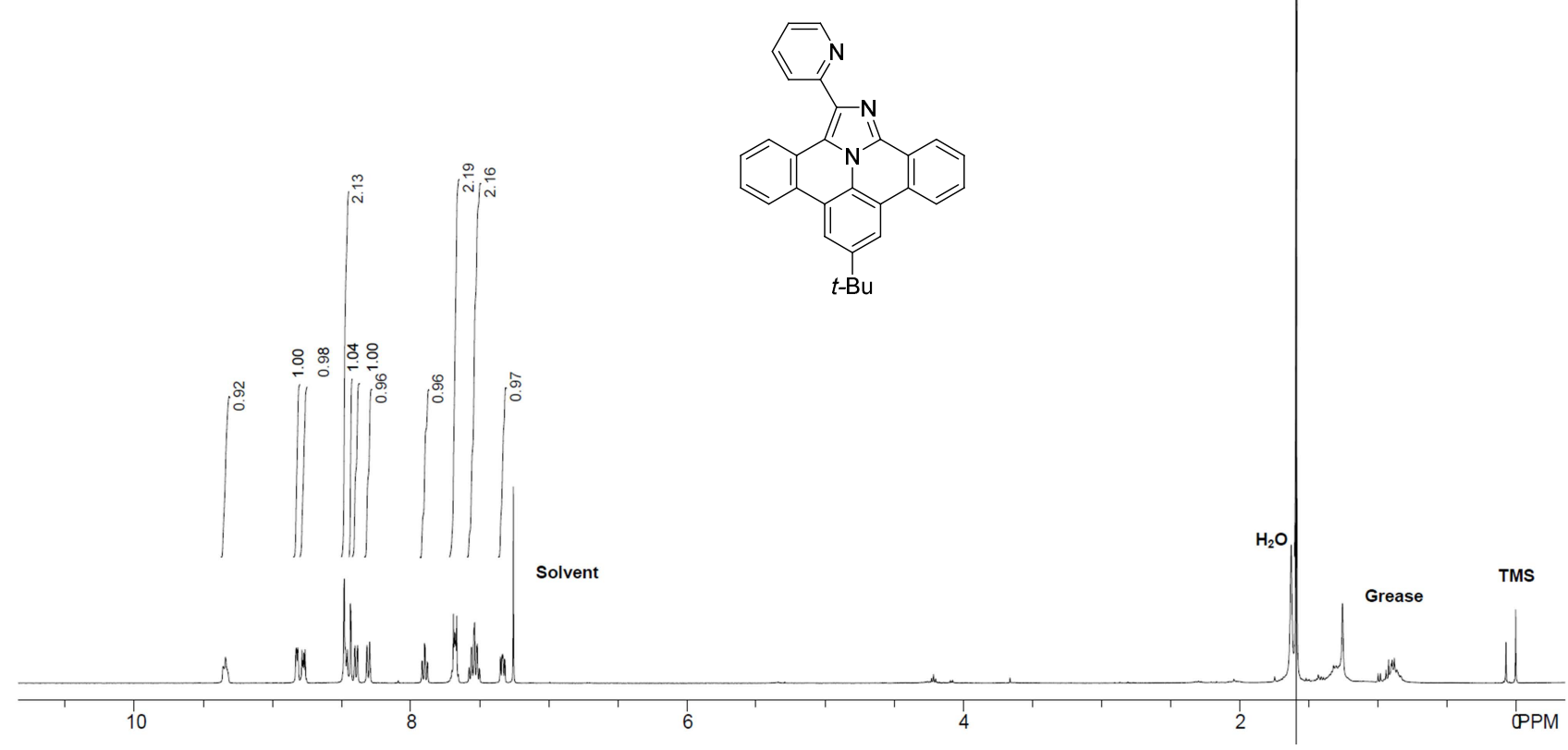

Figure S23. ${ }^{1} \mathrm{H} \mathrm{NMR}$ spectrum of $\mathbf{3 l}\left(400 \mathrm{MHz}, \mathrm{CDCl}_{3}, 300 \mathrm{~K}\right)$.

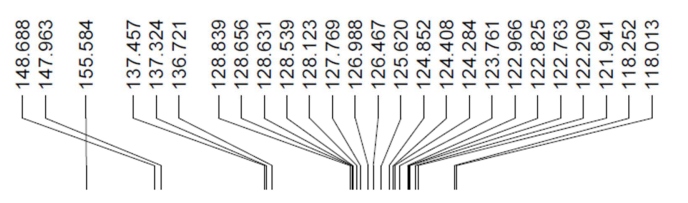

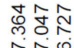

î́,

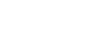

Solvent

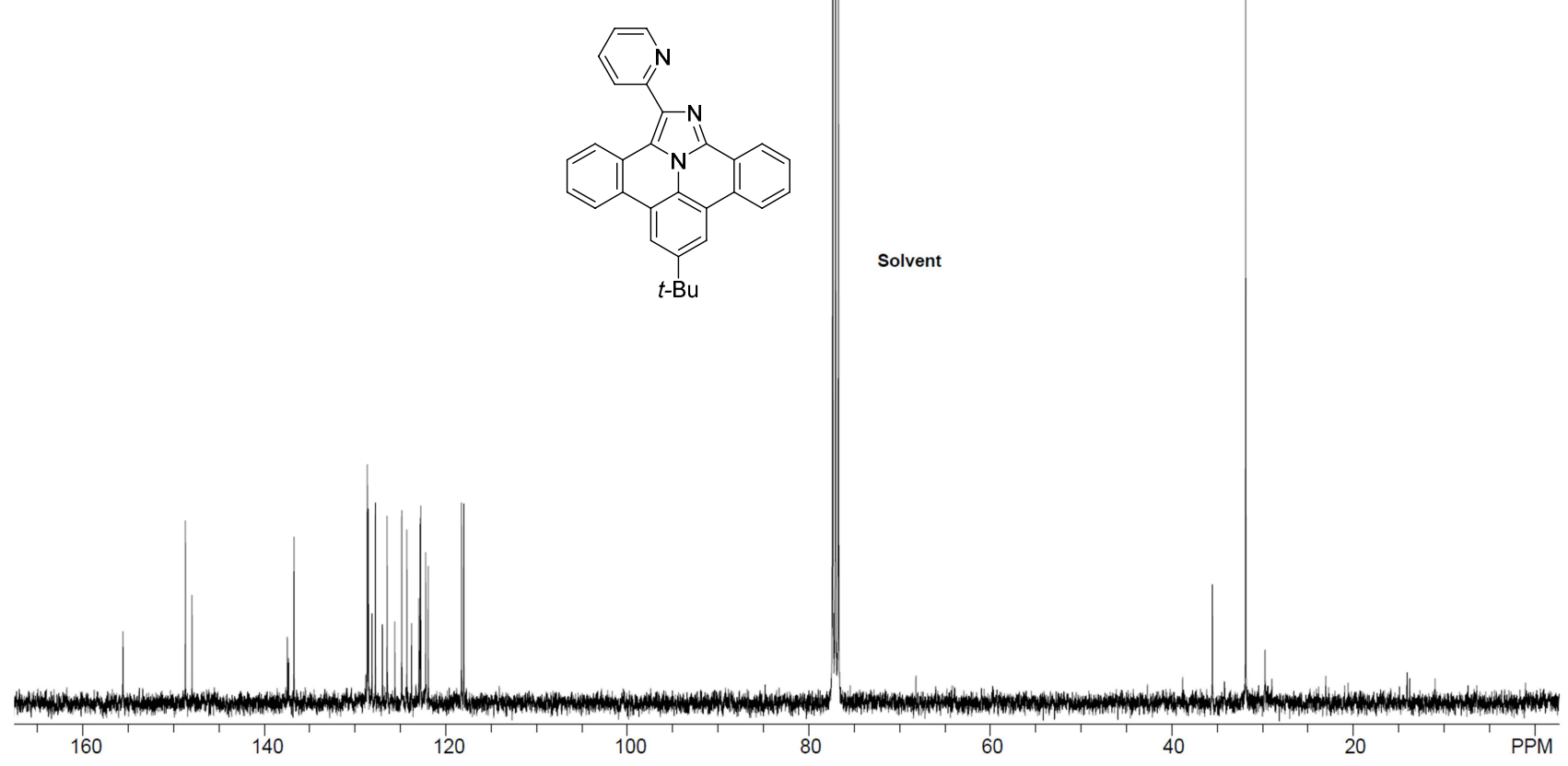

Figure S24. ${ }^{13} \mathrm{C}$ NMR spectrum of 31 (100 MHz, $\left.\mathrm{CDCl}_{3}, 300 \mathrm{~K}\right)$. 


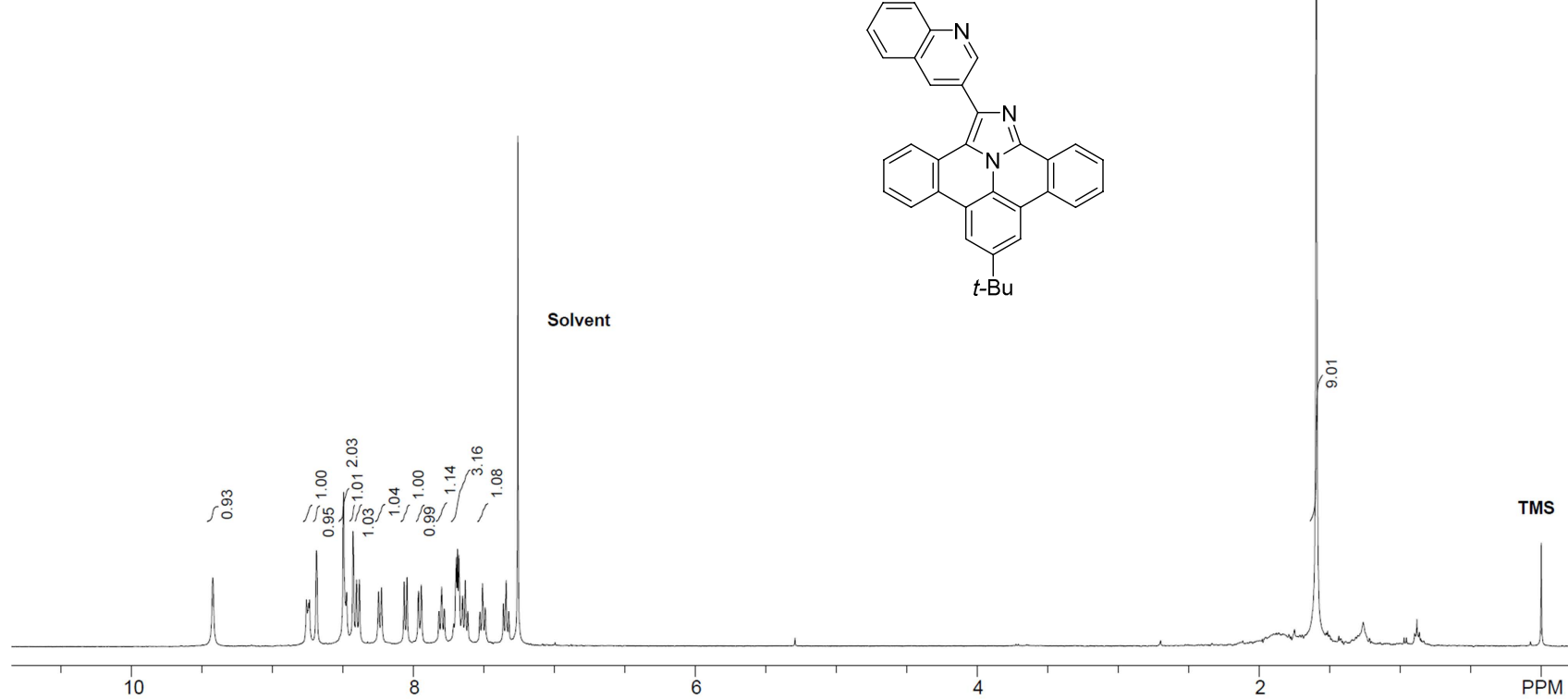

Figure S25. ${ }^{1} \mathrm{H}$ NMR spectrum of $\mathbf{3 m}\left(400 \mathrm{MHz}, \mathrm{CDCl}_{3}, 300 \mathrm{~K}\right)$.

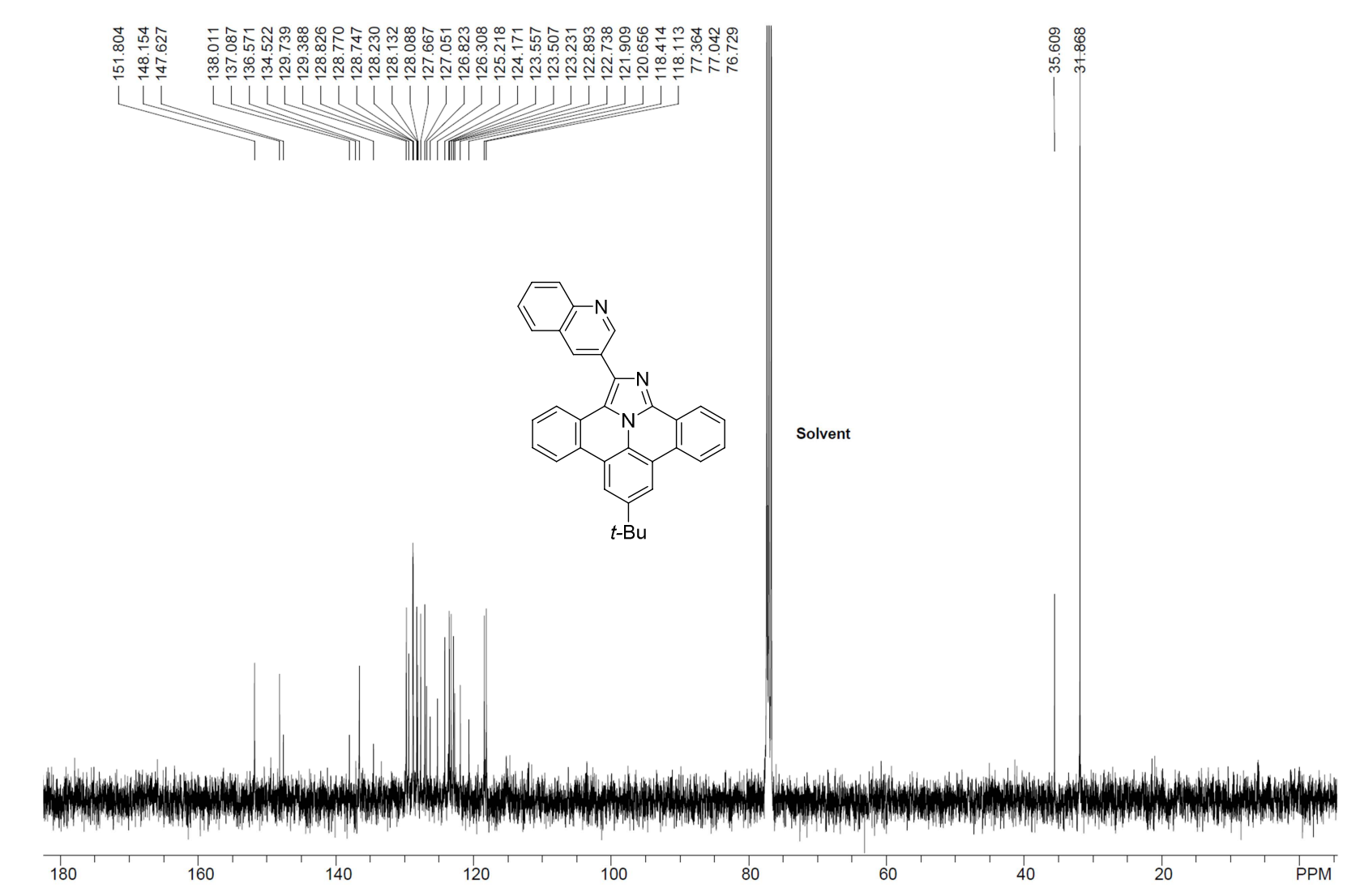

Figure S26. ${ }^{13} \mathrm{C}$ NMR spectrum of $\mathbf{3 m}\left(100 \mathrm{MHz}, \mathrm{CDCl}_{3}, 300 \mathrm{~K}\right)$. 


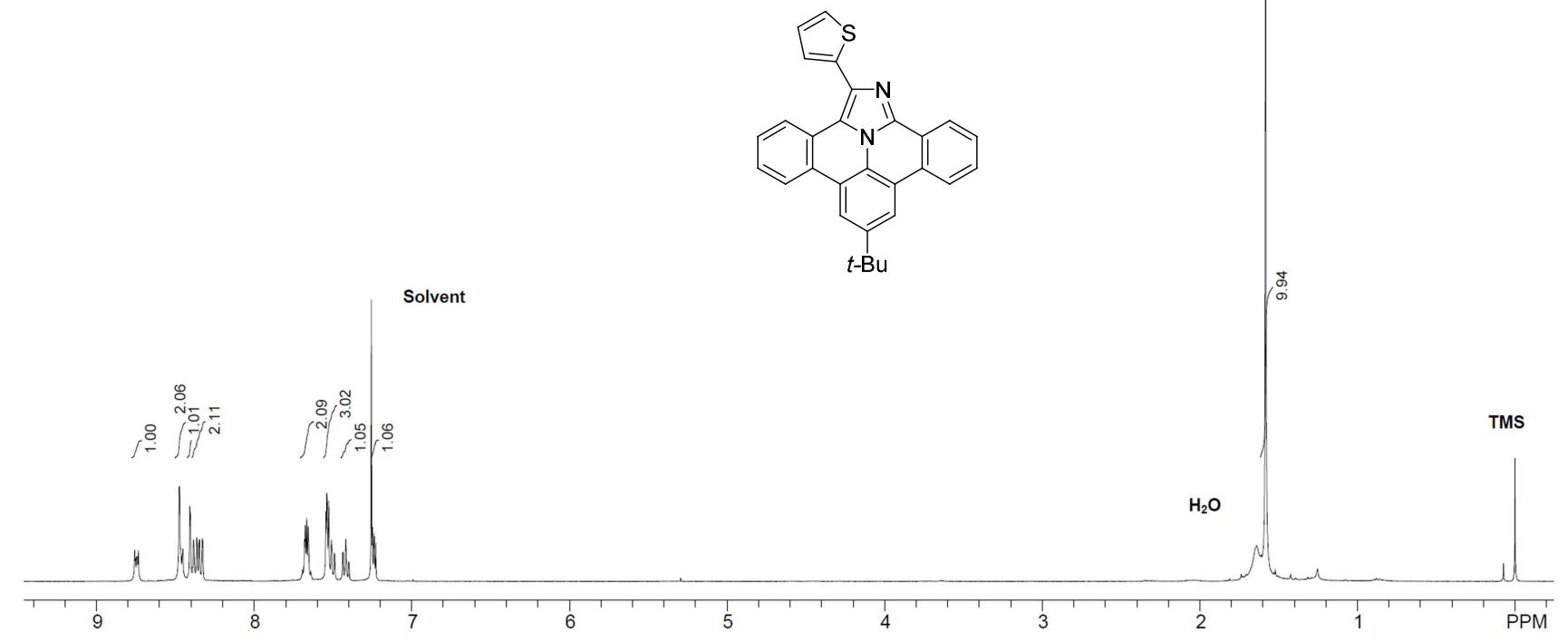

Figure S27. ${ }^{1} \mathrm{H} \mathrm{NMR}$ spectrum of $\mathbf{3 n}\left(400 \mathrm{MHz}, \mathrm{CDCl}_{3}, 300 \mathrm{~K}\right)$.

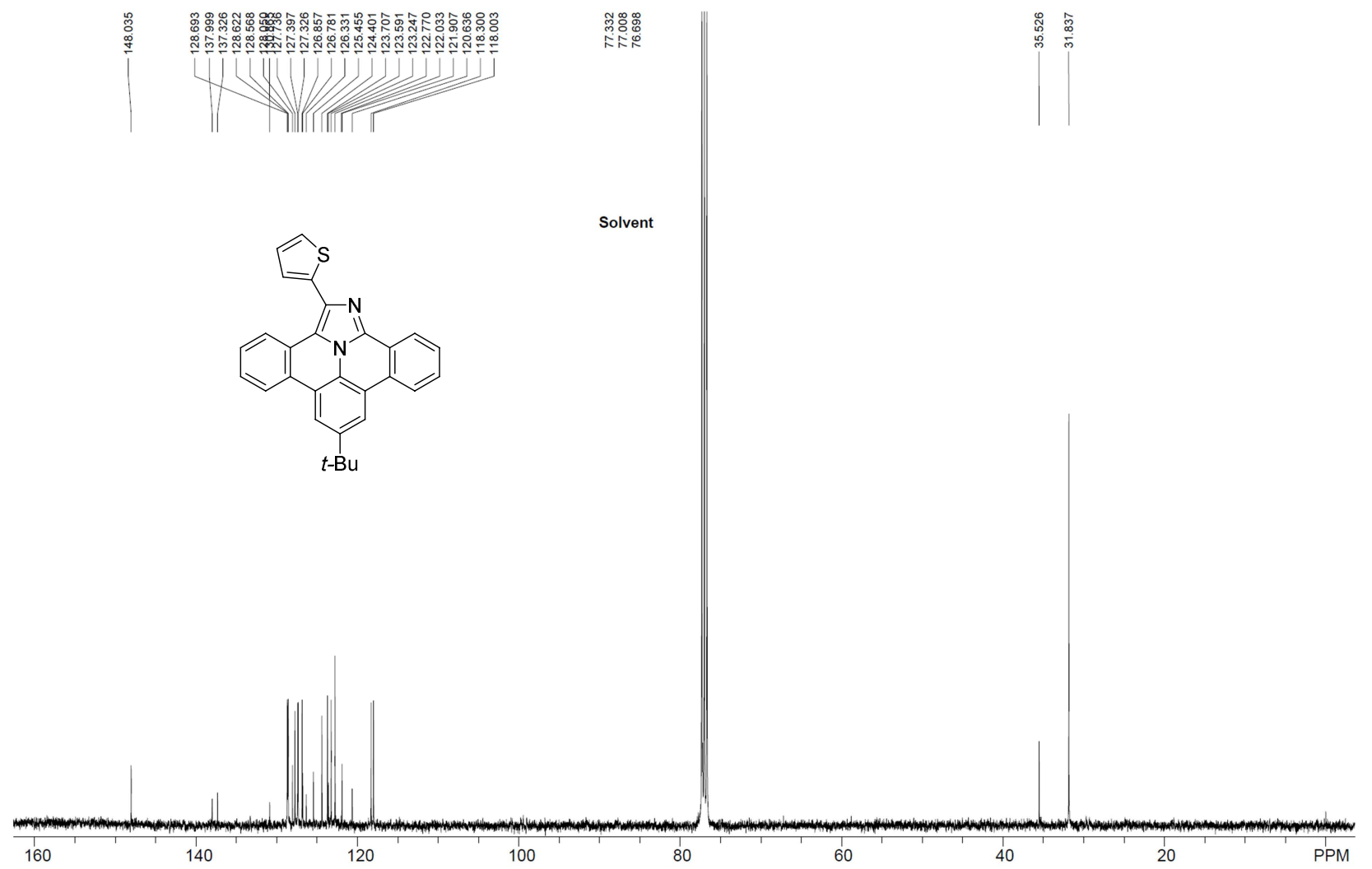

Figure S28. ${ }^{13} \mathrm{C}$ NMR spectrum of $\mathbf{3 n}\left(100 \mathrm{MHz}, \mathrm{CDCl}_{3}, 300 \mathrm{~K}\right)$. 

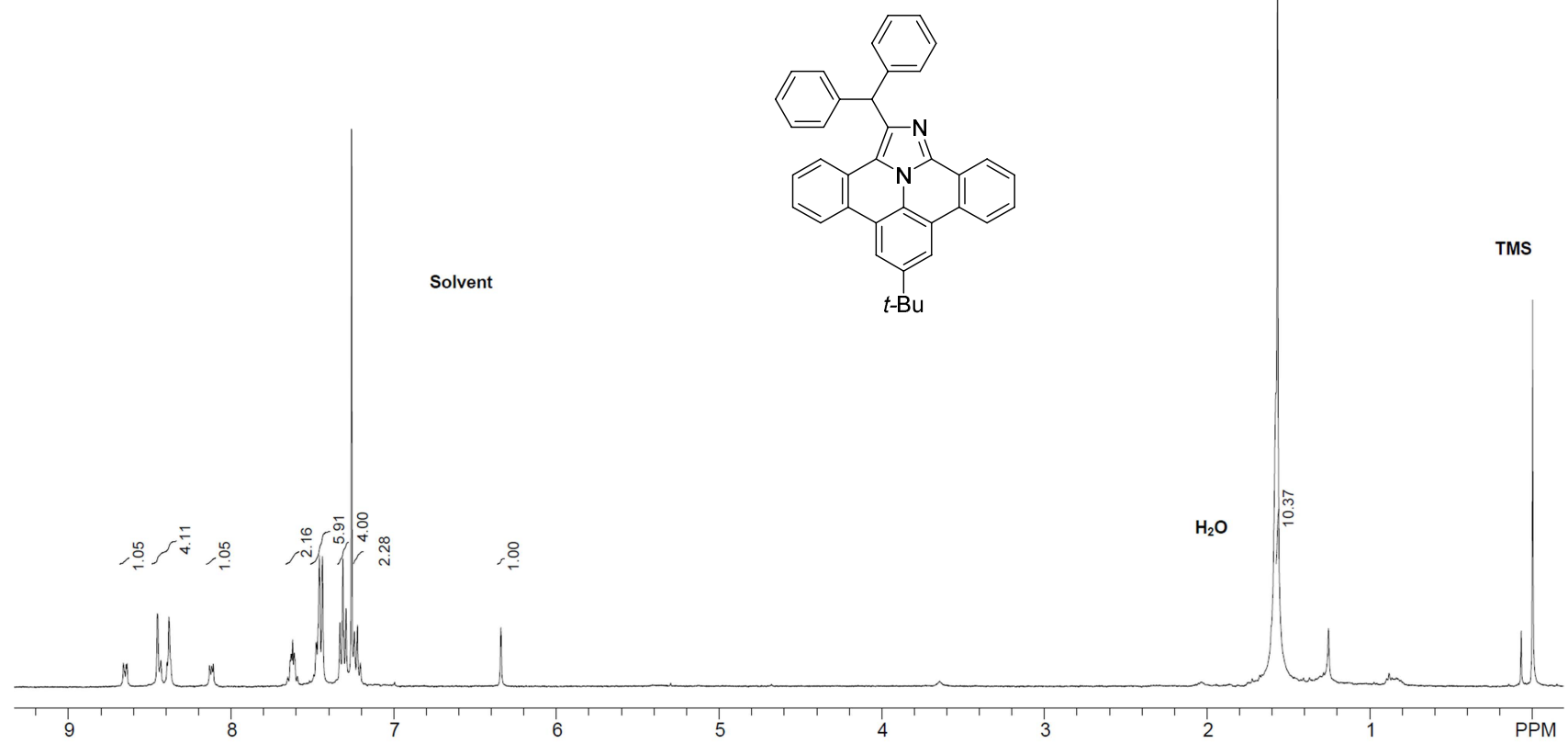

Figure S29. ${ }^{1} \mathrm{H} \mathrm{NMR}$ spectrum of $3 \mathbf{3 o}\left(400 \mathrm{MHz}, \mathrm{CDCl}_{3}, 300 \mathrm{~K}\right)$.

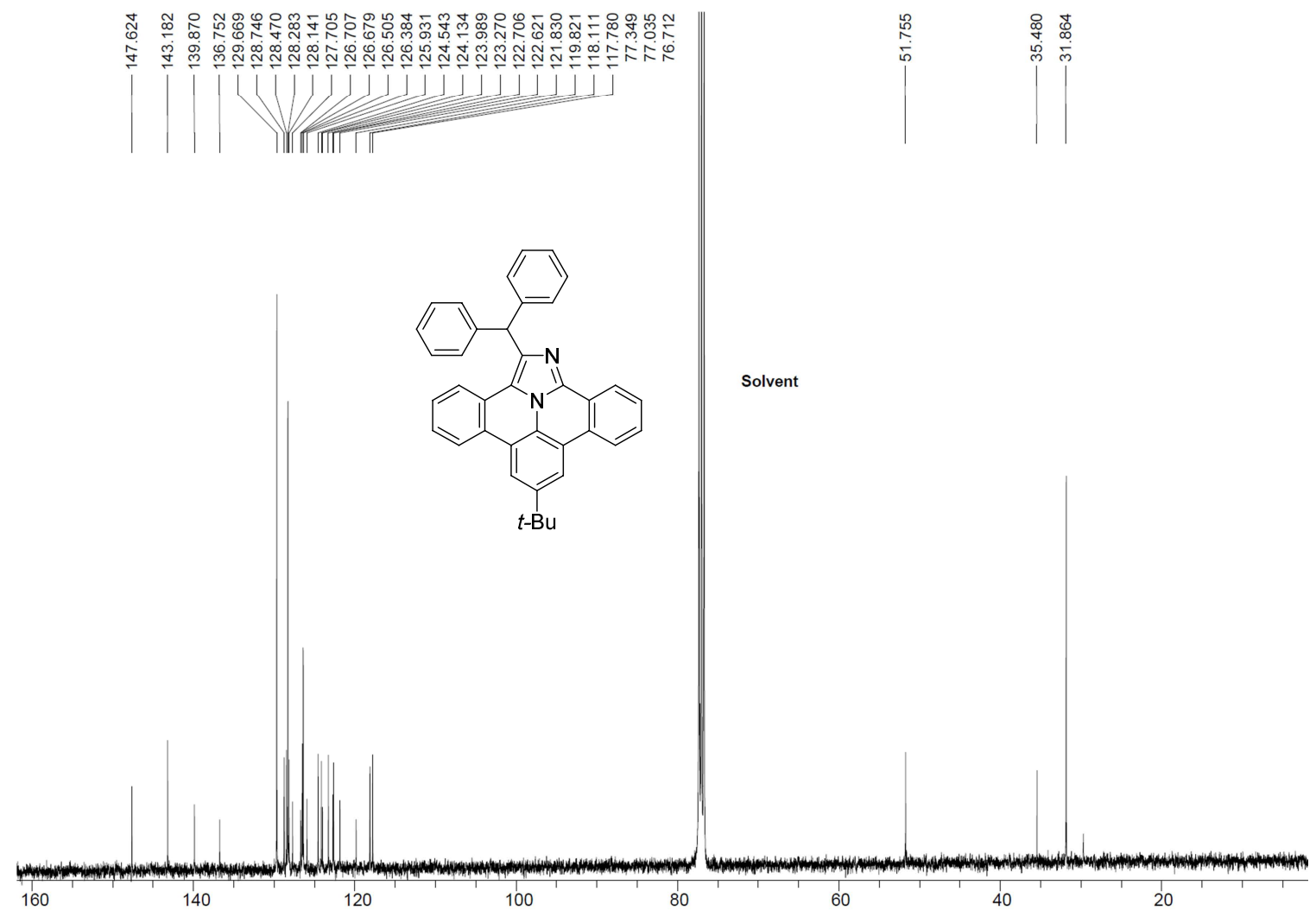

Figure S30. ${ }^{13} \mathrm{C}$ NMR spectrum of $30\left(100 \mathrm{MHz}, \mathrm{CDCl}_{3}, 300 \mathrm{~K}\right)$. 


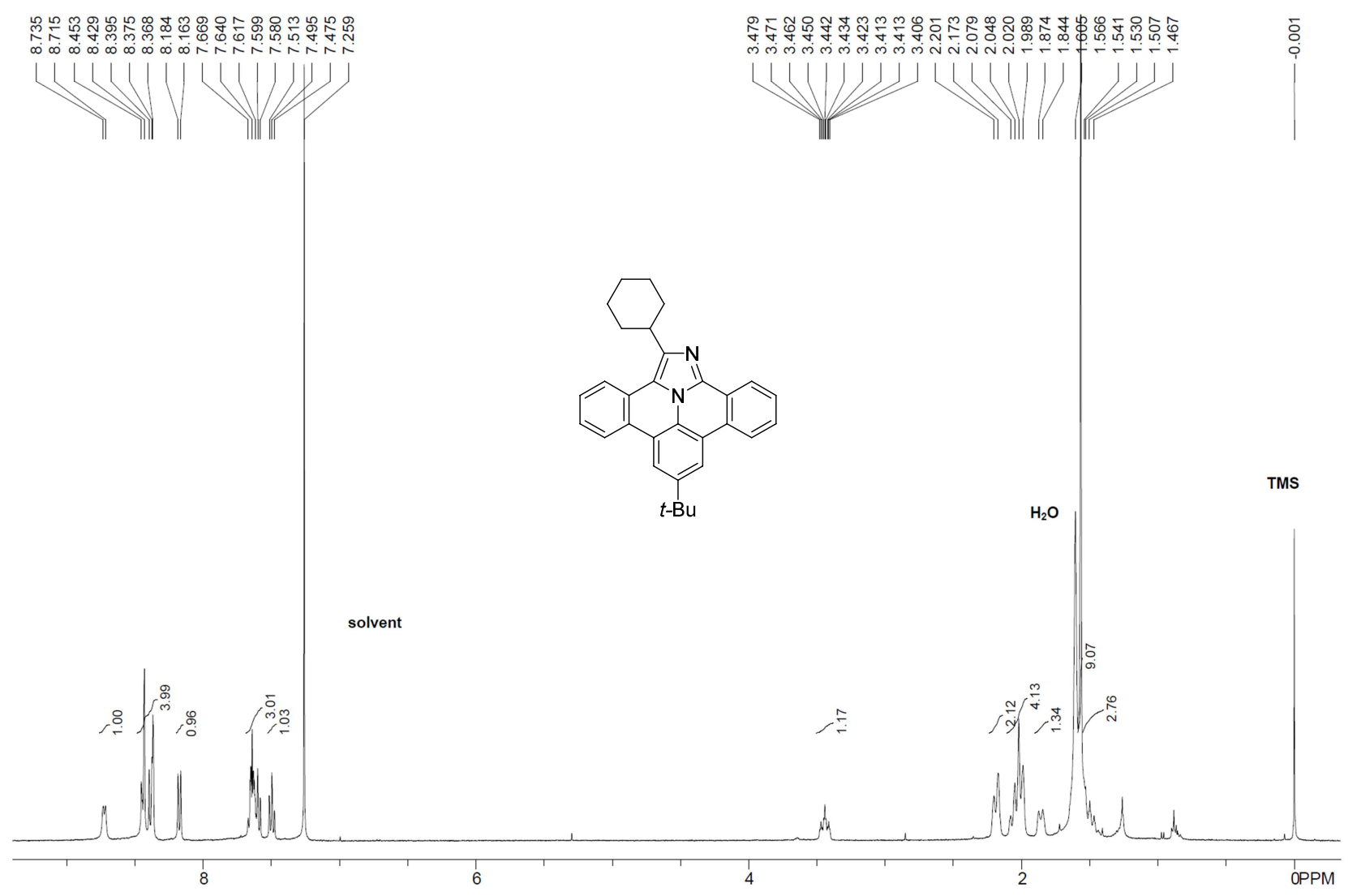

Figure S31. ${ }^{1} \mathrm{H}$ NMR spectrum of $\mathbf{3 p}\left(400 \mathrm{MHz}, \mathrm{CDCl}_{3}, 300 \mathrm{~K}\right)$.

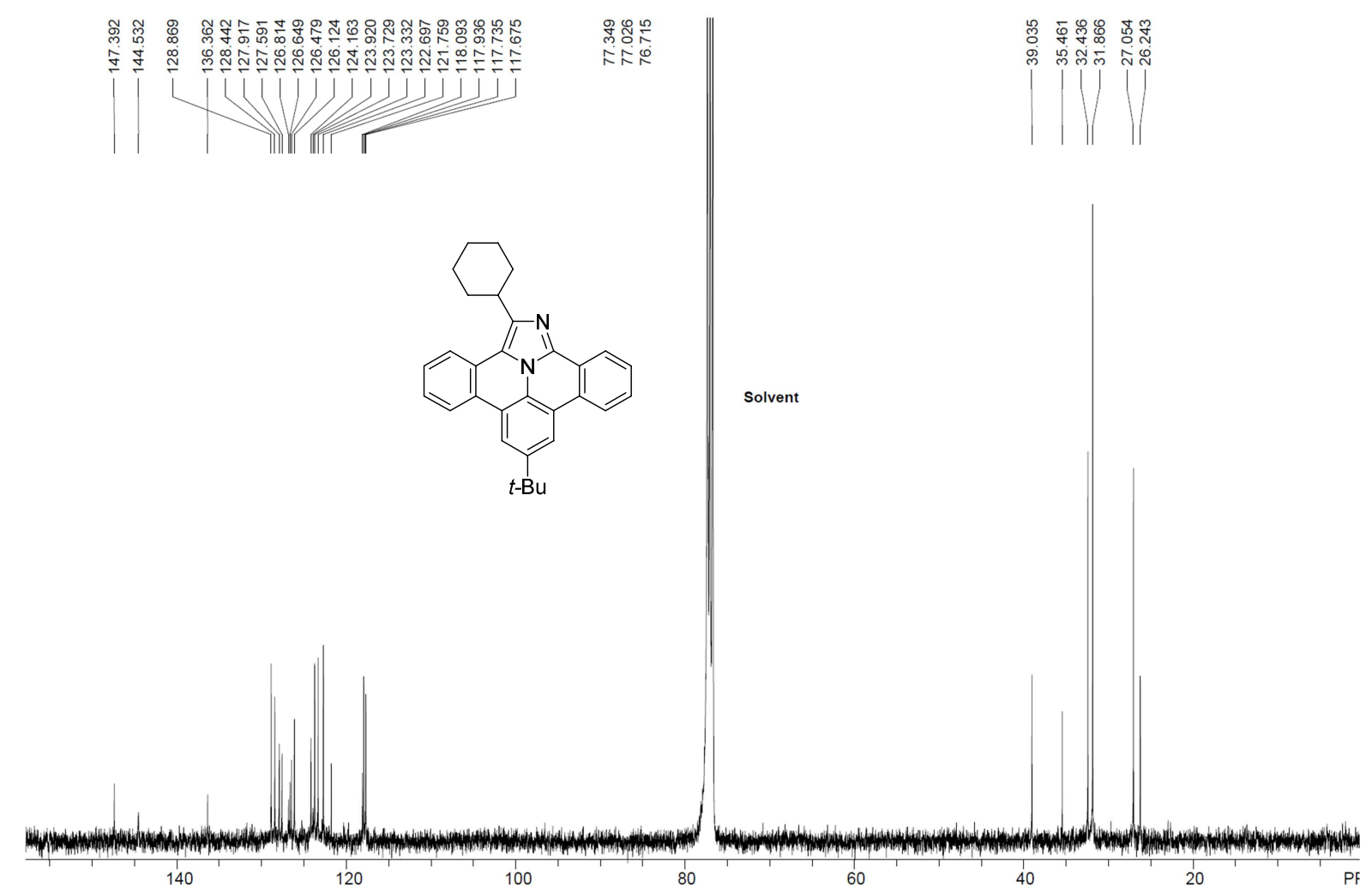

Figure 32. ${ }^{13} \mathrm{C}$ NMR spectrum of $\mathbf{3 p}\left(100 \mathrm{MHz}, \mathrm{CDCl}_{3}, 300 \mathrm{~K}\right)$. 


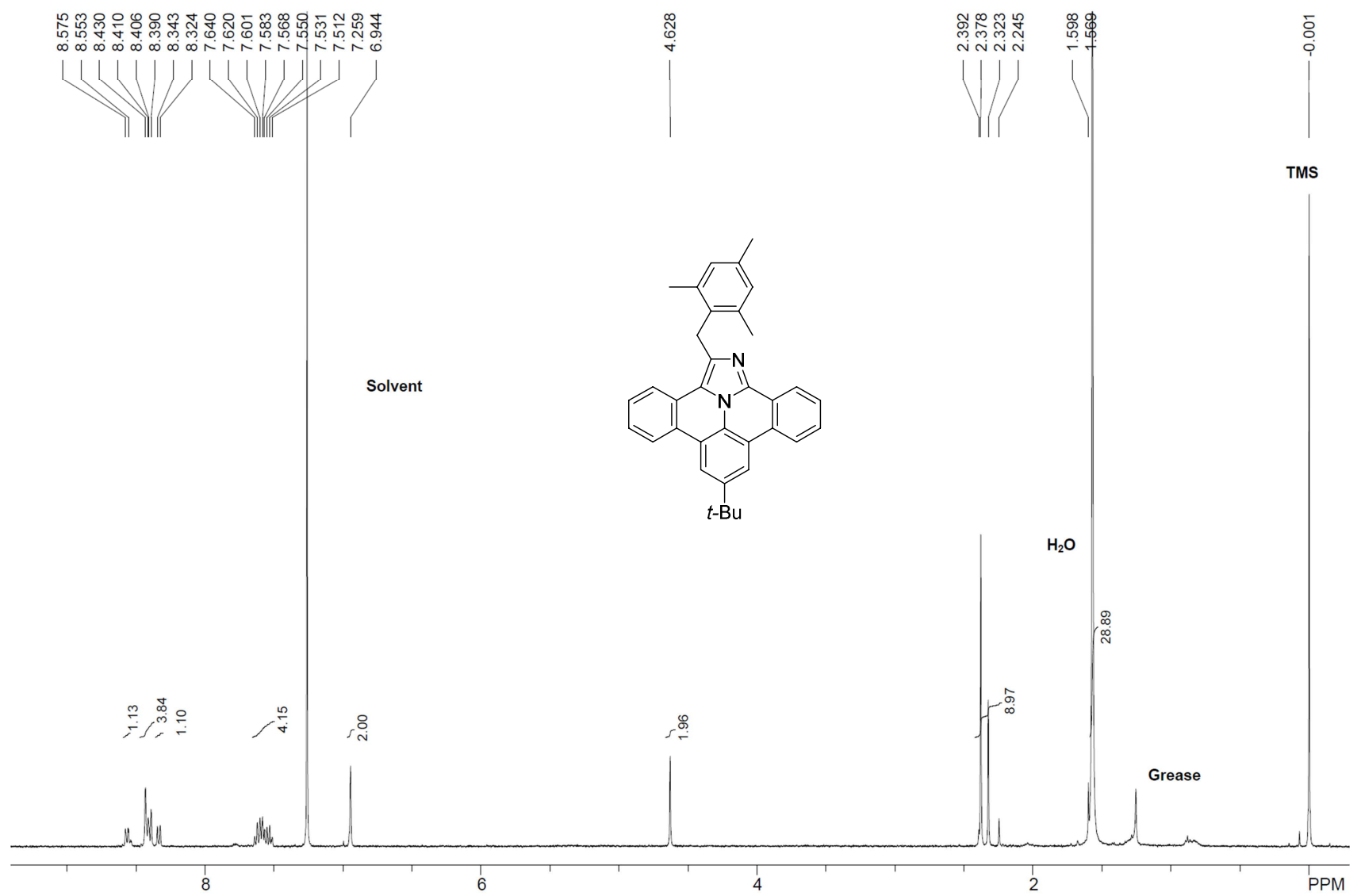

Figure S33. ${ }^{1} \mathrm{H} \mathrm{NMR}$ spectrum of $\mathbf{3 q}\left(400 \mathrm{MHz}, \mathrm{CDCl}_{3}, 300 \mathrm{~K}\right)$.
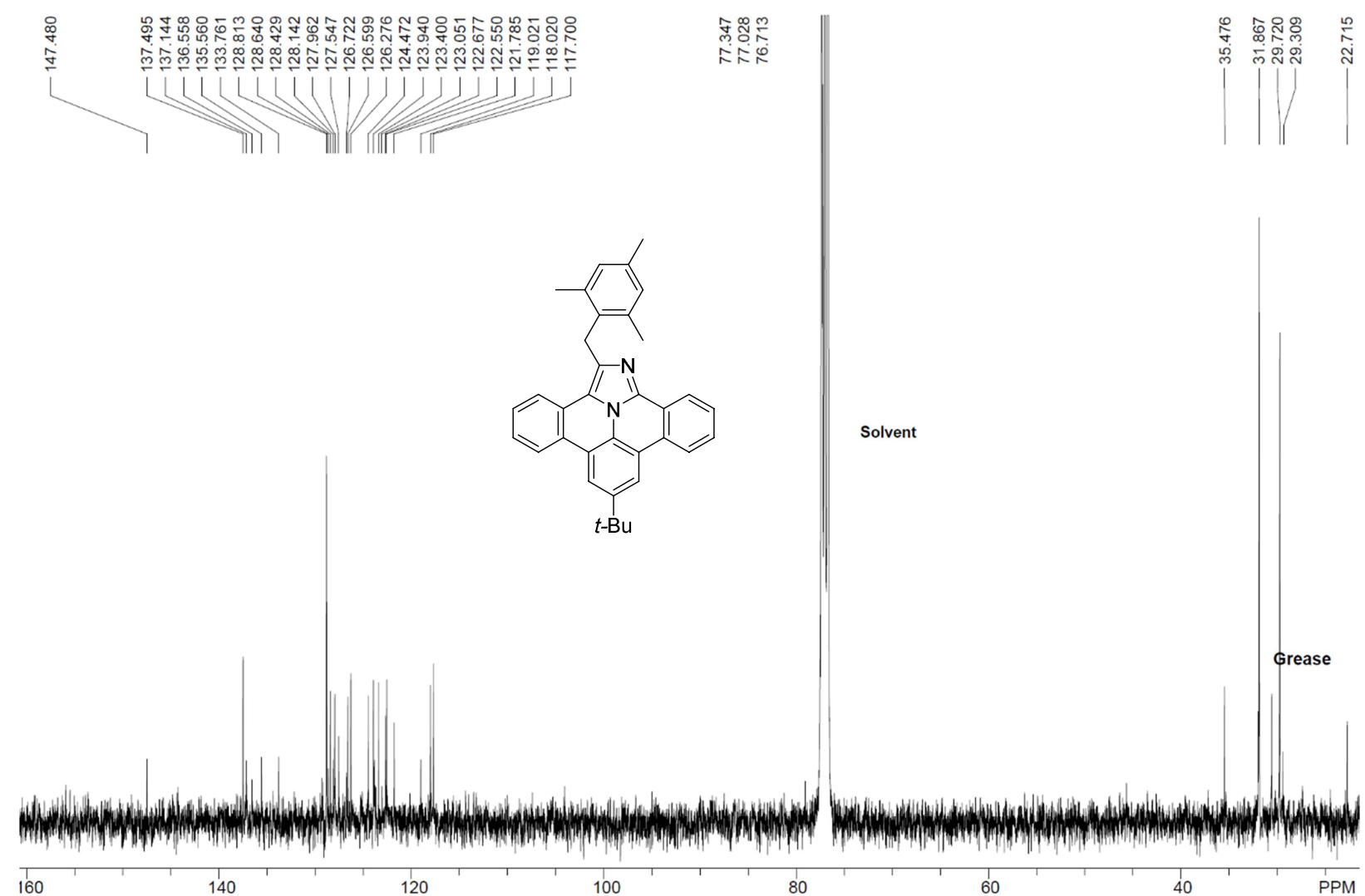

Figure S34. ${ }^{13} \mathrm{C}$ NMR spectrum of $\mathbf{3 q}\left(100 \mathrm{MHz}, \mathrm{CDCl}_{3}, 300 \mathrm{~K}\right)$. 

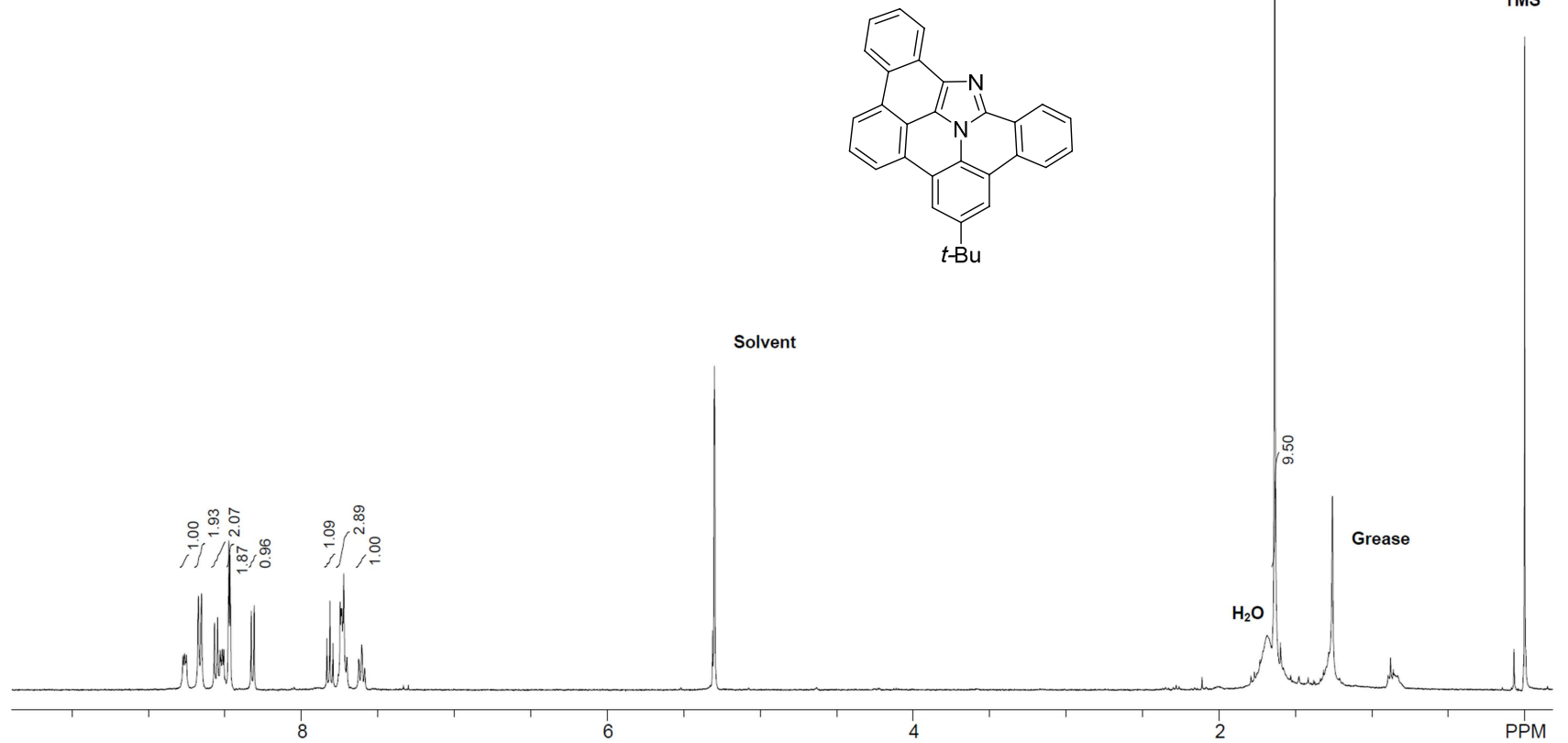

Figure S35. ${ }^{1} \mathrm{H}$ NMR spectrum of $4 \mathbf{a}\left(400 \mathrm{MHz}, \mathrm{CD}_{2} \mathrm{Cl}_{2} / \mathrm{CS}_{2}, 300 \mathrm{~K}\right)$.

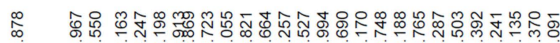

ơ

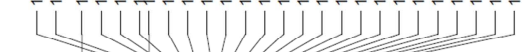

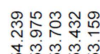
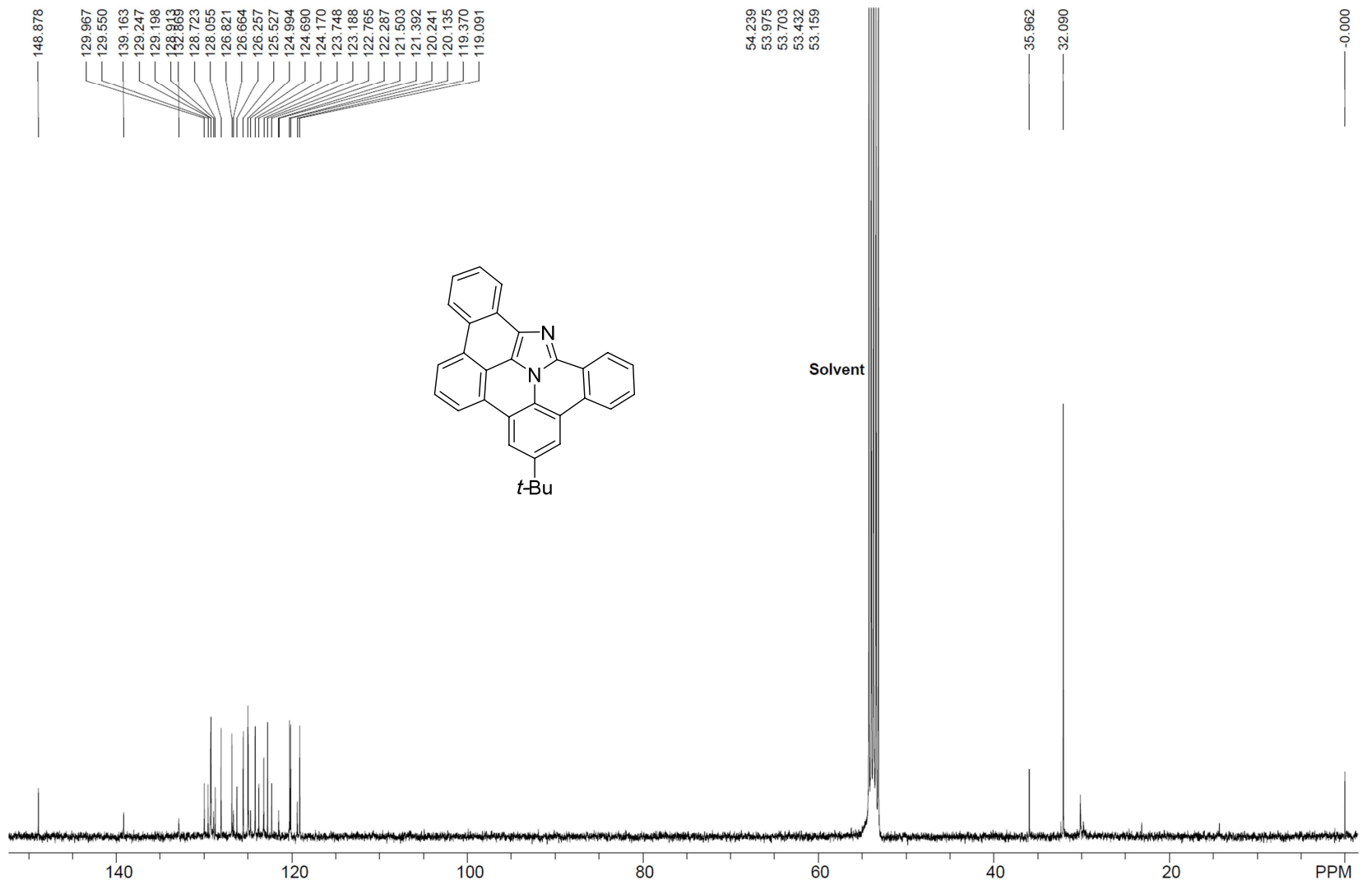

1 1 | $\mid$ |

Figure S36. ${ }^{13} \mathrm{C}$ NMR spectrum of $4 \mathbf{a}\left(100 \mathrm{MHz}, \mathrm{CD}_{2} \mathrm{Cl}_{2} / \mathrm{CS}_{2}, 300 \mathrm{~K}\right)$. 

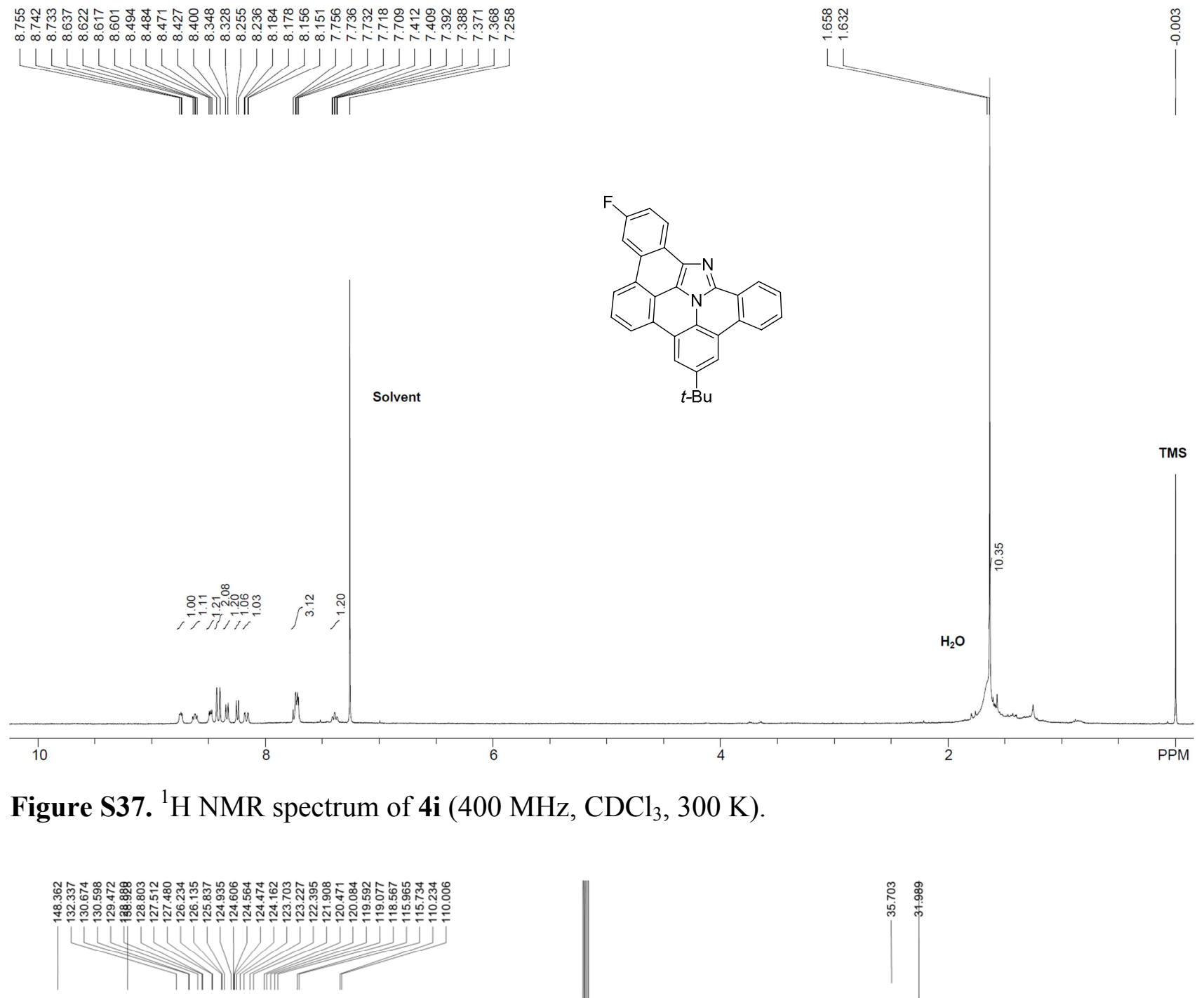

Figure S37. ${ }^{1} \mathrm{H}$ NMR spectrum of $\mathbf{4 i}\left(400 \mathrm{MHz}, \mathrm{CDCl}_{3}, 300 \mathrm{~K}\right)$.

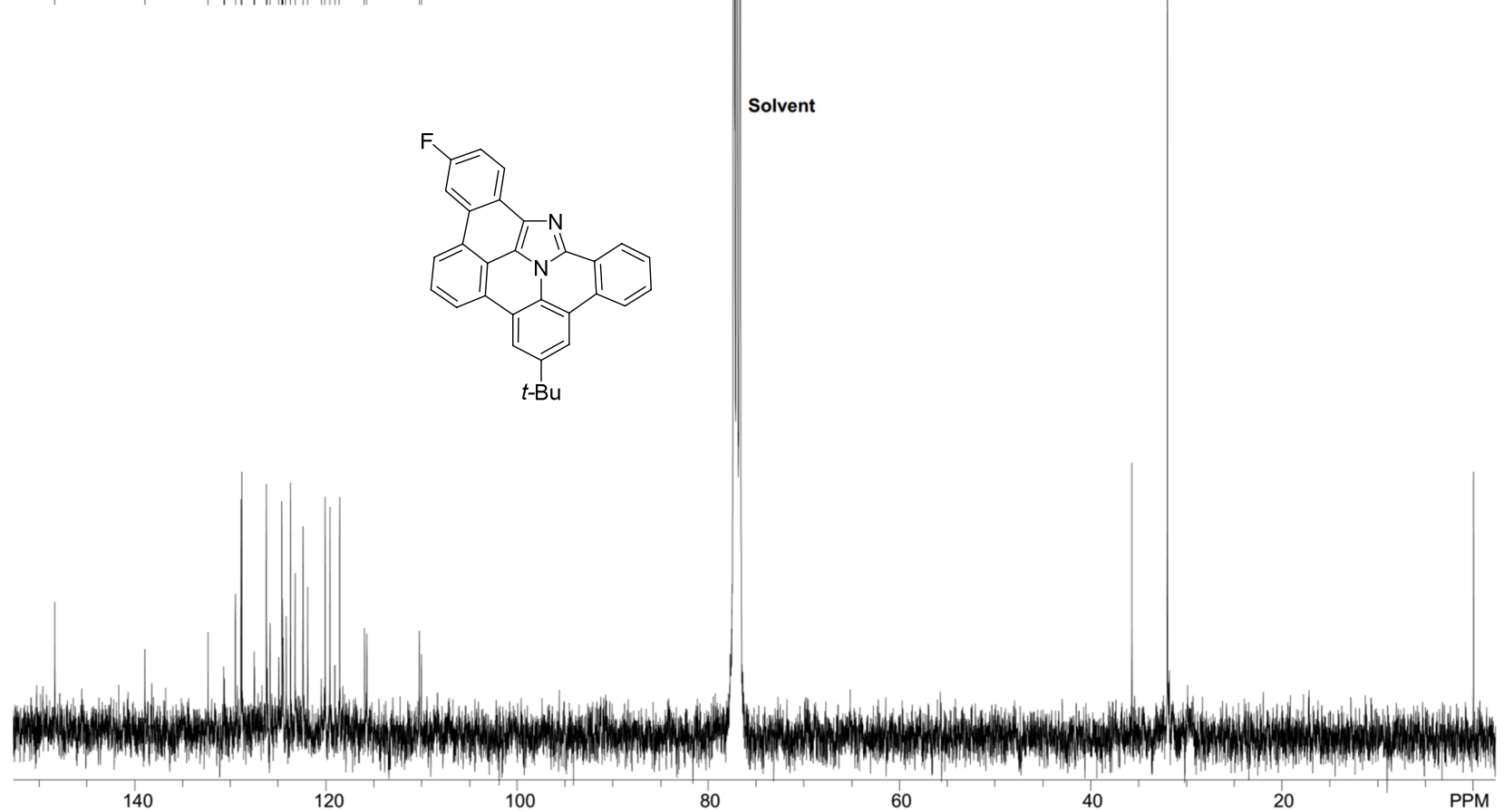

Figure S38. ${ }^{13} \mathrm{C}$ NMR spectrum of $4 \mathbf{i}\left(100 \mathrm{MHz}, \mathrm{CDCl}_{3}, 300 \mathrm{~K}\right)$. 


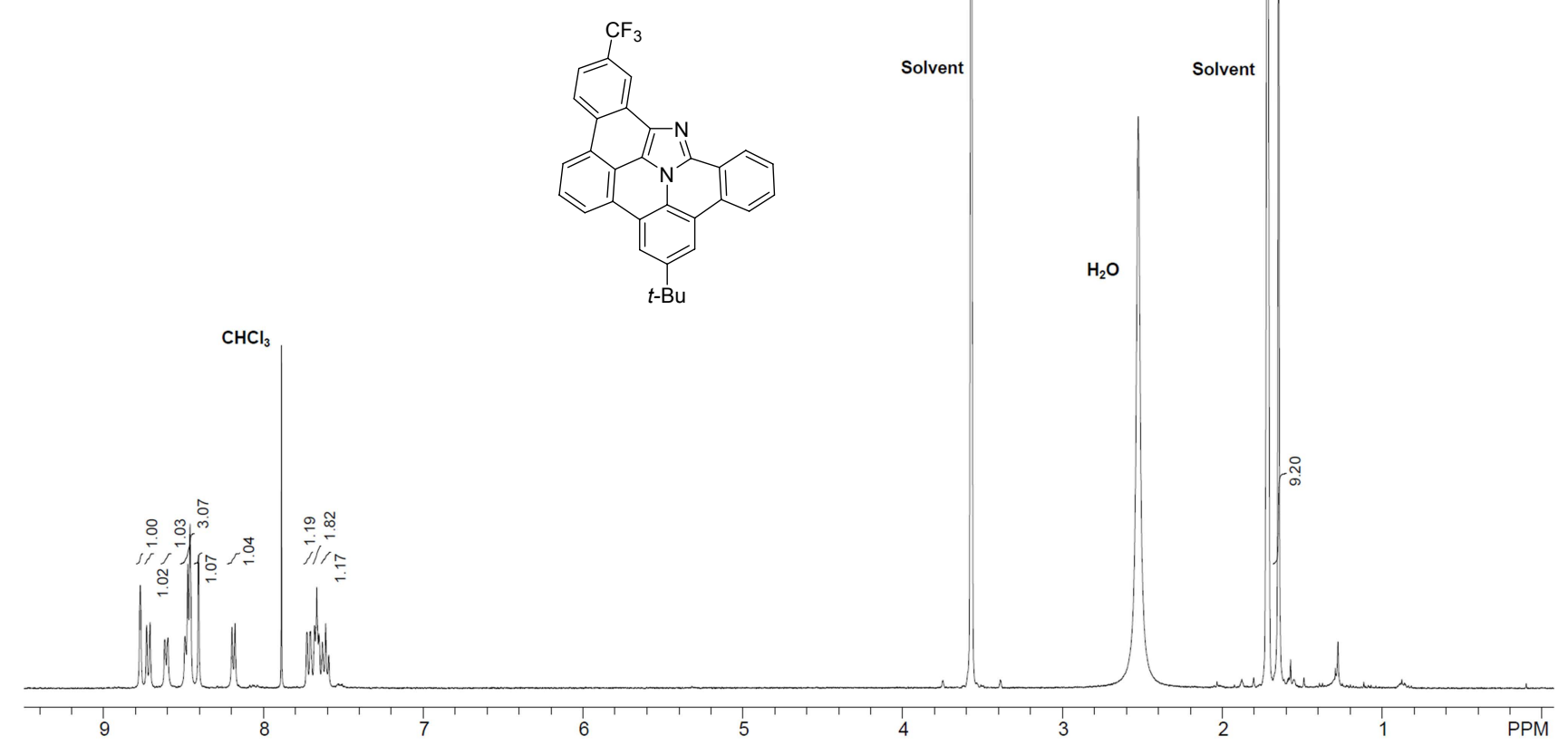

Figure S39. ${ }^{1} \mathrm{H}$ NMR spectrum of $\mathbf{4 j}$ ( $\left.400 \mathrm{MHz}, \mathrm{THF}-d_{8}, 300 \mathrm{~K}\right)$.

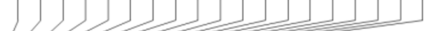

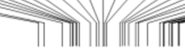
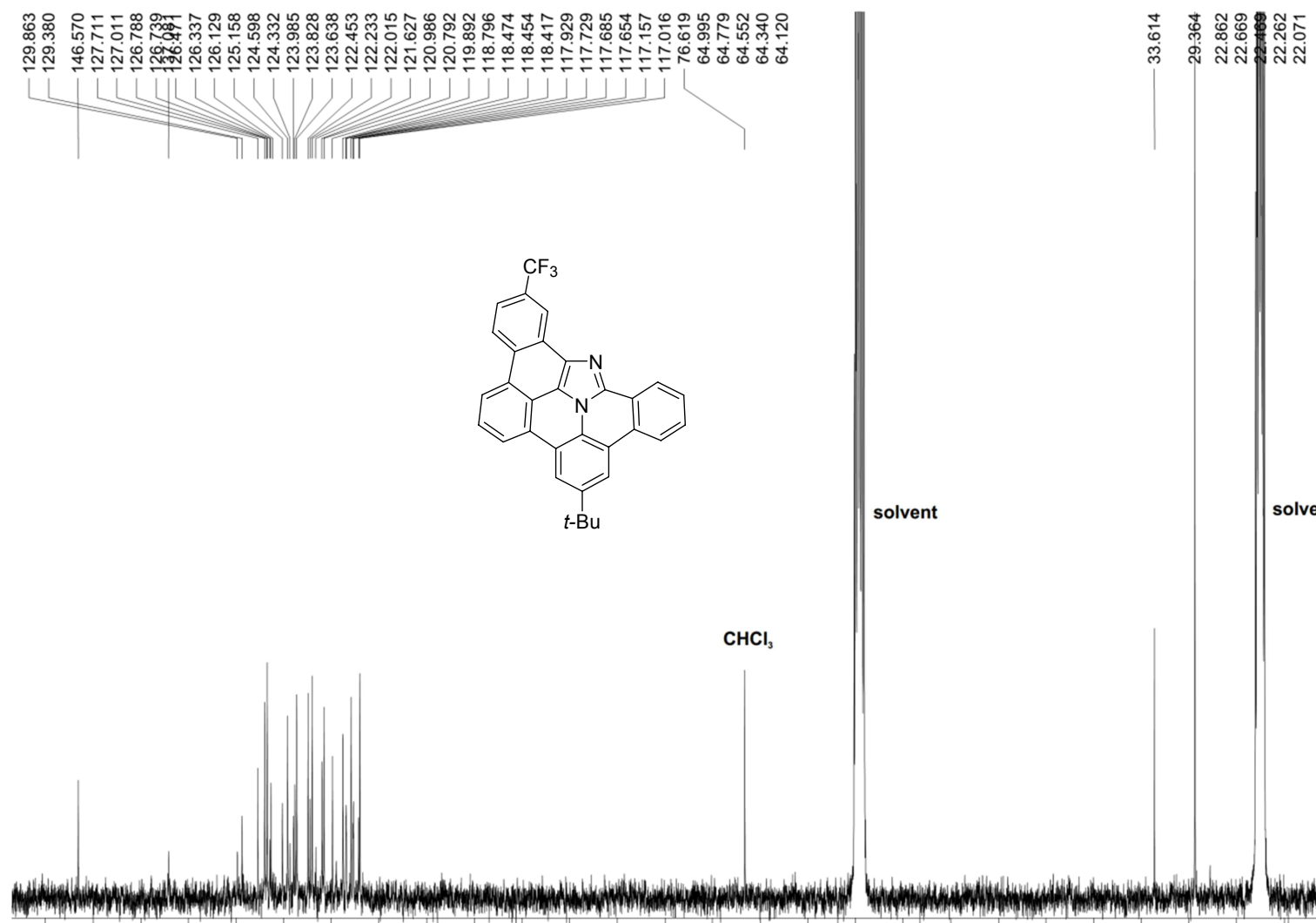

$\mathrm{CHCl}_{3}$

Figure S40. ${ }^{13} \mathrm{C}$ NMR spectrum of $\mathbf{4 j}\left(100 \mathrm{MHz}, \mathrm{THF}-d_{8}, 300 \mathrm{~K}\right)$. 


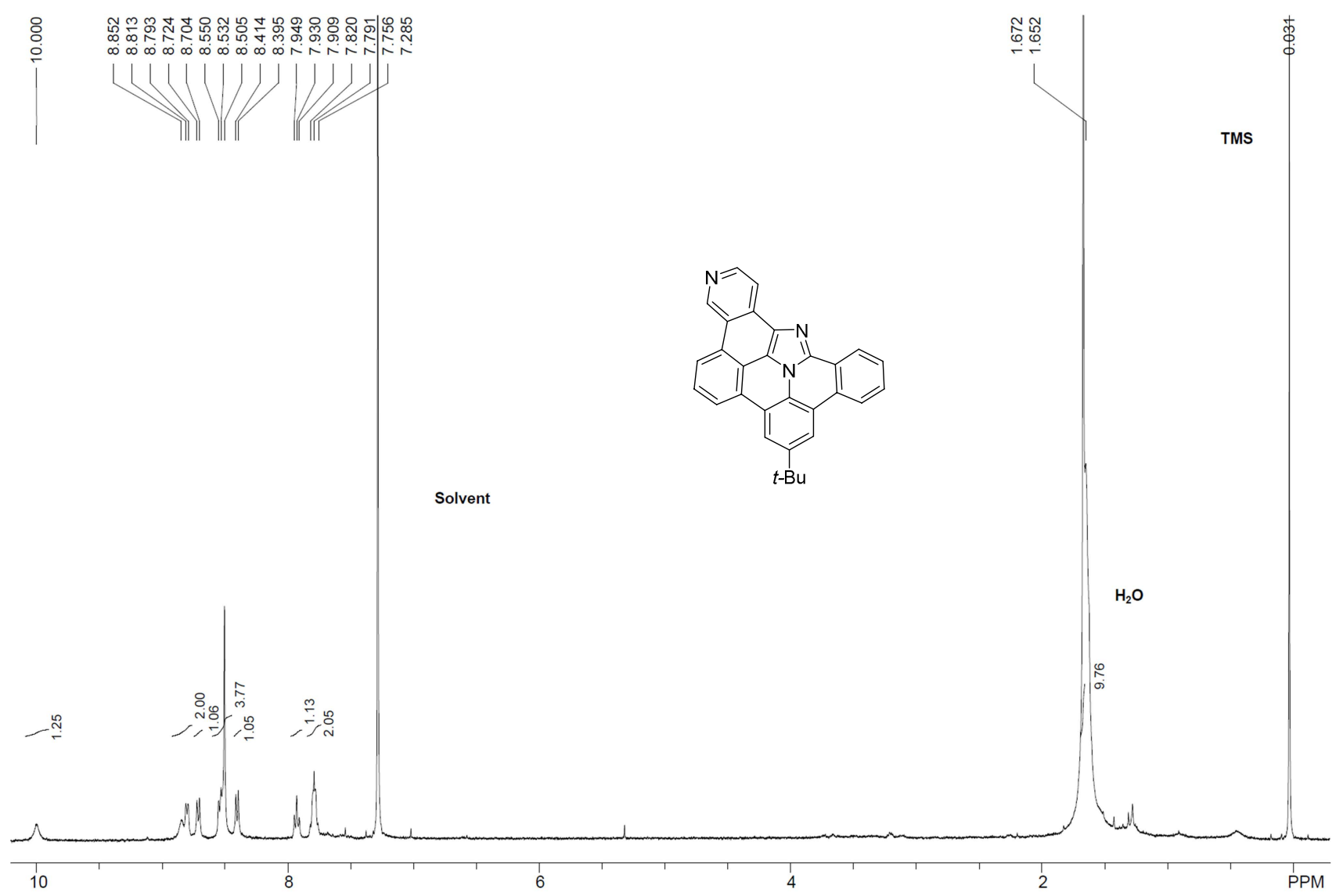

Figure S41. ${ }^{1} \mathrm{H} \mathrm{NMR}$ spectrum of $\mathbf{4 k}\left(400 \mathrm{MHz}, \mathrm{CDCl}_{3}, 300 \mathrm{~K}\right)$.
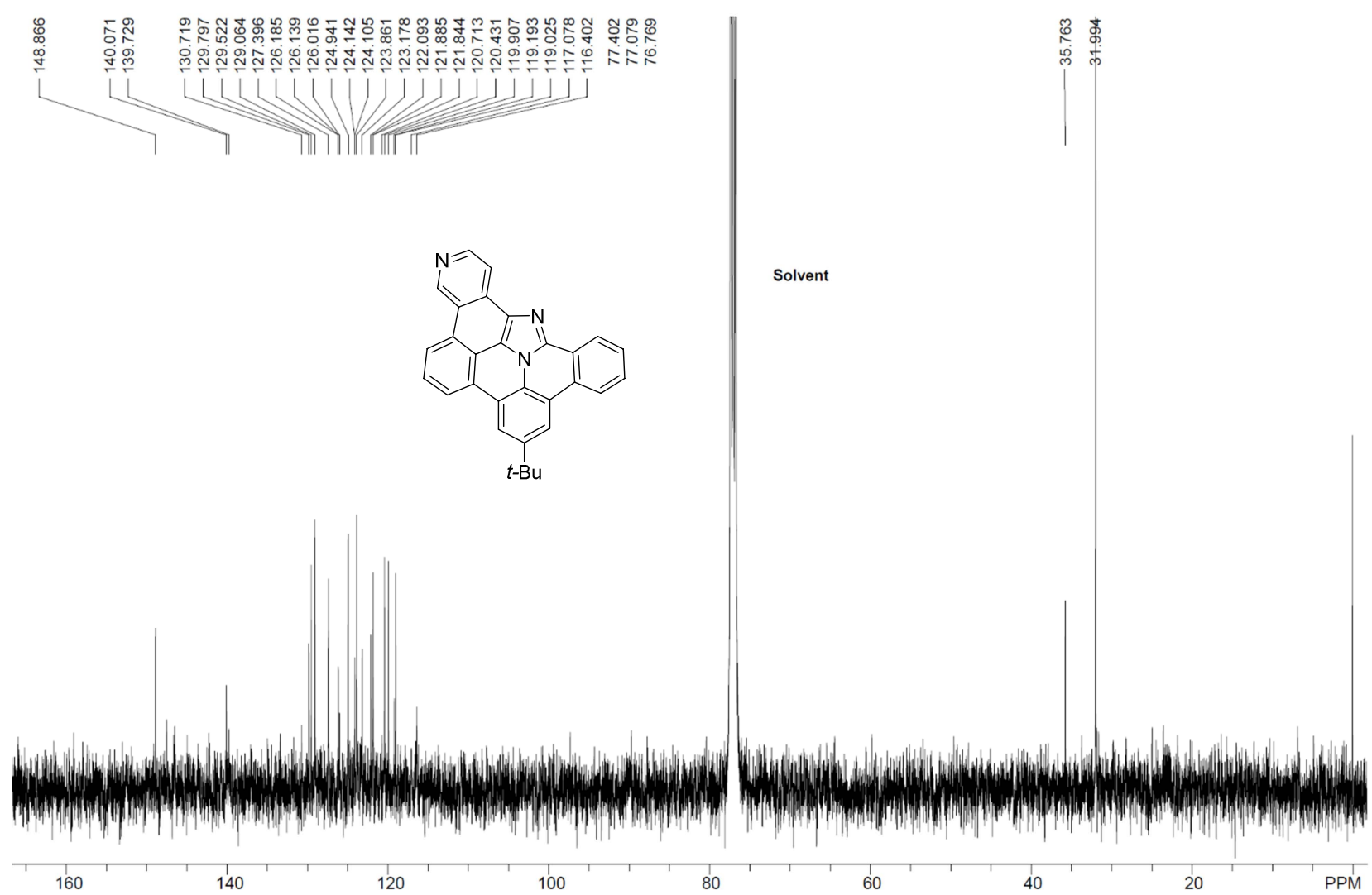

Figure S42. ${ }^{13} \mathrm{C}$ NMR spectrum of $\mathbf{4 k}\left(100 \mathrm{MHz}, \mathrm{CDCl}_{3}, 300 \mathrm{~K}\right)$. 


\section{X-Ray Crystallographic Data}

Single crystals suitable for X-ray analysis for $\mathbf{3 g}$, $\mathbf{4 a}$ and $\mathbf{4 k}$ were obtained by slow diffusion of methanol to a solution of $\mathbf{3 g}$ or $\mathbf{4 a}$ in dichloromethane, and a solution of $\mathbf{4 k}$ in chloroform, respectively, under air at room temperature. A single crystal was mounted with mineral oil on a loop-type mount and transferred to the goniometer of a Bruker B8 Quest diffractometer. The radiation was performed with Multilayer Mirror-monochromated Incoatec microfocus source $(\lambda=$ $0.71073 \AA$ ). The structures were solved by direct method with SHELXT-2014/5 $5^{3}$ and refined by fullmatrix least-squares techniques against $F^{2}$ with SHELXL-2018/3. ${ }^{3}$ The intensities were corrected for Lorentz and polarization effects. The non-hydrogen atoms were refined anisotropically. Hydrogen atoms were placed using AFIX instructions.

Table S2. Crystal data and structure refinement for $\mathbf{3 g}$, $\mathbf{4 a}$, and $\mathbf{4 k}$.

\begin{tabular}{|c|c|c|c|}
\hline compound & $3 \mathbf{g}$ & $4 a$ & $4 \mathbf{k}$ \\
\hline CCDC number & 2013728 & 1997008 & 1997009 \\
\hline Molecular formula & $\mathrm{C}_{31} \mathrm{H}_{25} \mathrm{~N}_{3} \cdot 0.5 \mathrm{CH}_{2} \mathrm{Cl}_{2}$ & $\mathrm{C}_{31} \mathrm{H}_{22} \mathrm{~N}_{2} \cdot \mathrm{CH}_{3} \mathrm{OH}$ & $\begin{array}{l}\mathrm{C}_{30} \mathrm{H}_{21} \mathrm{~N}_{3} 2 \mathrm{CHCl}_{3} \\
0.5 \mathrm{CH}_{3} \mathrm{OH}\end{array}$ \\
\hline Formula weight & 482.00 & 454.55 & 678.25 \\
\hline Temperature $(\mathrm{K})$ & 100 & 100 & 100 \\
\hline Wavelength $(\AA)$ & 0.71073 & 0.71073 & 0.71073 \\
\hline Crystal system & orthorhombic & monoclinic & orthorhombic \\
\hline Space group & Pca2 $_{1}$ & $P 2_{1} / c$ & Pbca \\
\hline Unit cell dimensions a $(\AA)$ & $31.3702(17)$ & $12.0043(5)$ & $6.7271(6)$ \\
\hline $\mathrm{b}(\AA)$ & $6.9008(4)$ & $26.4715(11)$ & $29.439(2)$ \\
\hline$c(\AA)$ & 22.1586 & $6.8245(2)$ & $31.222(3)$ \\
\hline$\alpha\left(^{\circ}\right)$ & 90 & 90 & 90 \\
\hline$\beta\left(^{\circ}\right)$ & 90 & $91.660(2)$ & 90 \\
\hline$\gamma\left(\left(^{\circ}\right)\right.$ & 90 & 90 & 90 \\
\hline Volume $\left(\AA^{3}\right)$ & $4796.9(4)$ & 2167.72(14) & $6183.2(9)$ \\
\hline Z & 8 & 4 & 8 \\
\hline Density (calculated) $\left(\mathrm{mg} \cdot \mathrm{m}^{-3}\right)$ & 1.335 & 1.393 & 1.457 \\
\hline Absorption cosfficient $\left(\mathrm{mm}^{-1}\right)$ & 0.186 & 0.084 & 0.586 \\
\hline$F(000)$ & 2024 & 960 & 2776 \\
\hline Crystal size $\left(\mathrm{mm}^{3}\right)$ & $0.020 \times 0.120 \times 0.280$ & $0.220 \times 0.040 \times 0.020$ & $0.020 \times 0.030 \times 0.240$ \\
\hline Theta range $\left({ }^{\circ}\right)$ & 2.75 to 25.63 & 2.87 to 30.72 & 2.61 to 25.36 \\
\hline \multirow[t]{3}{*}{ Index ranges } & $-32<=\mathrm{h}<=38$ & $-17<=\mathrm{h}<=17$ & $0<=\mathrm{h}<=8$ \\
\hline & $-8<=k<=7$ & $0<=\mathrm{k}<=37$ & $0<=k<=35$ \\
\hline & $-26<=\mid<=18$ & $0<=\mid<=9$ & $0<=\mid<=37$ \\
\hline Reflections collected & 20266 & 6615 & 5525 \\
\hline Min. and max. transmission & $0.9500,0.9960$ & $0.9820,0.9980$ & $0.8720,0.9880$ \\
\hline Data / restraints / parameters & 7745 / 777 / 692 & $6615 / 65 / 343$ & 5525 / 656 / 468 \\
\hline Goodness-of-fit on $\mathrm{F}^{2}$ & 1.073 & 1.035 & 1.100 \\
\hline \multirow[t]{2}{*}{ Final $R$ indices $[/>2 \sigma(I)]$} & $R_{1}=0.0874$ & $R_{1}=0.0866$ & $R_{1}=0.1223$ \\
\hline & $\mathrm{w} R_{2}=0.1982$ & $w R_{2}=0.20653$ & $\mathrm{w} R_{2}=0.2951$ \\
\hline \multirow[t]{2}{*}{$R$ indices (all data) $[/>2 \sigma(I)]$} & $R_{1}=0.1390$ & $R_{1}=0.1936$ & $R_{1}=0.2035$ \\
\hline & $w R_{2}=0.2289$ & $w R_{2}=0.2683$ & $w R_{2}=0.3371$ \\
\hline Largest diff. peak and hole $\left(\mathrm{e} . \AA^{-3}\right)$ & $0.580,-0.502$ & $0.376,-0.665$ & $0.886,-0.573$ \\
\hline
\end{tabular}

(3) Sheldrick, G. M. University of Göttingen: Göttingen, Germany, 2014. 


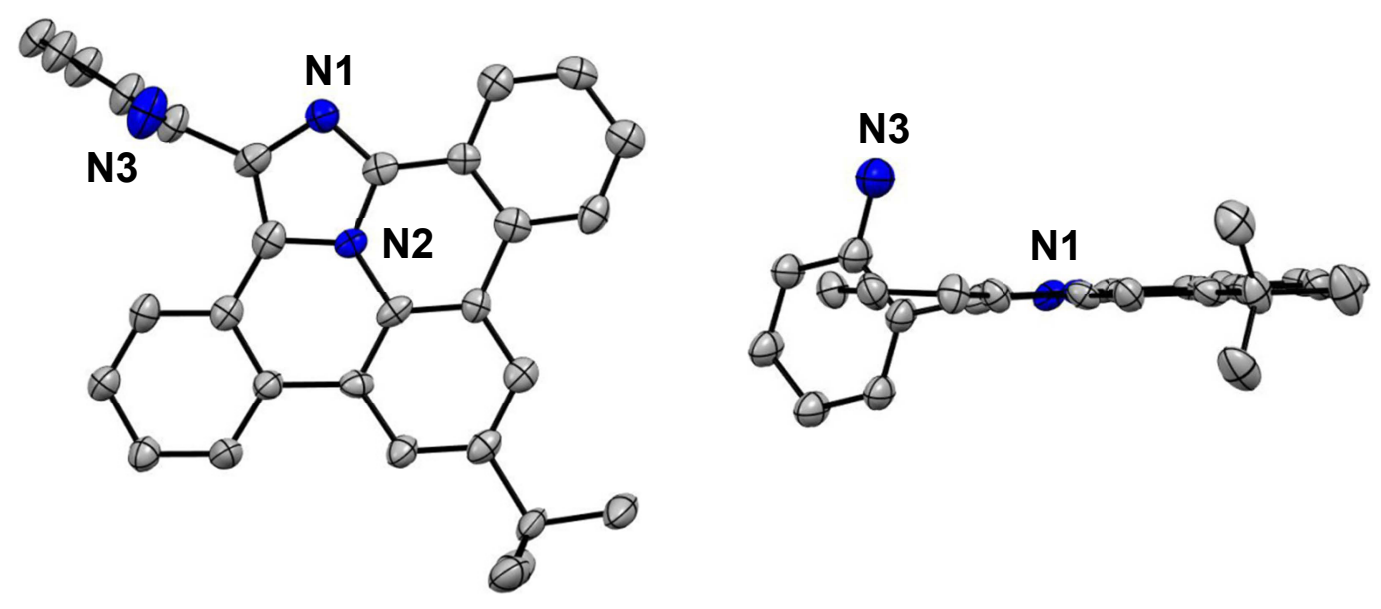

Figure S43. X-ray structures of $\mathbf{3 g}$ with thermal ellipsoids of $50 \%$ probability. Solvent molecules and hydrogen atoms were omitted for clarity.
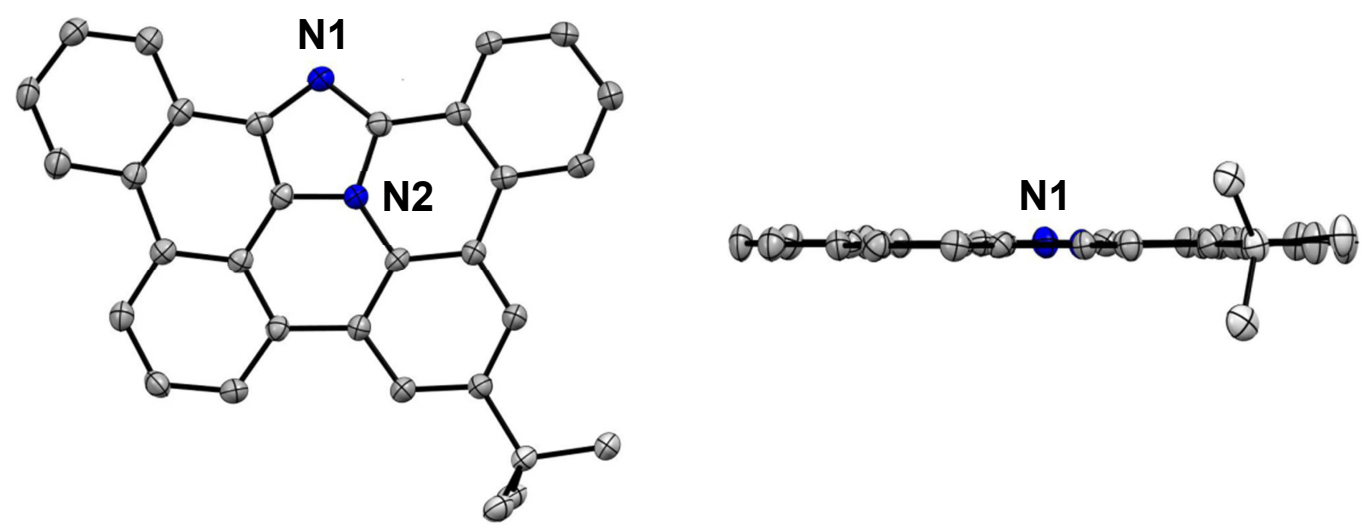

Figure S44. X-ray structures of 4 a with thermal ellipsoids of 50\% probability. Solvent molecules and hydrogen atoms were omitted for clarity.
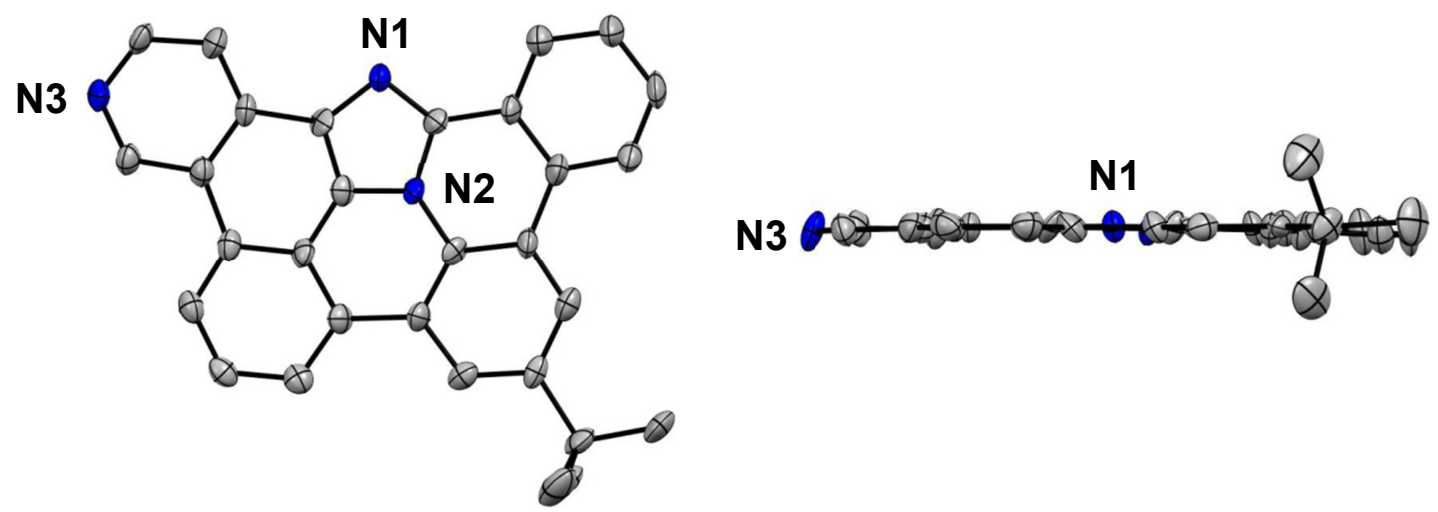

Figure S45. X-ray structures of $\mathbf{4 k}$ with thermal ellipsoids of $50 \%$ probability. Solvent molecules and hydrogen atoms were omitted for clarity. 


\section{Optical Properties}
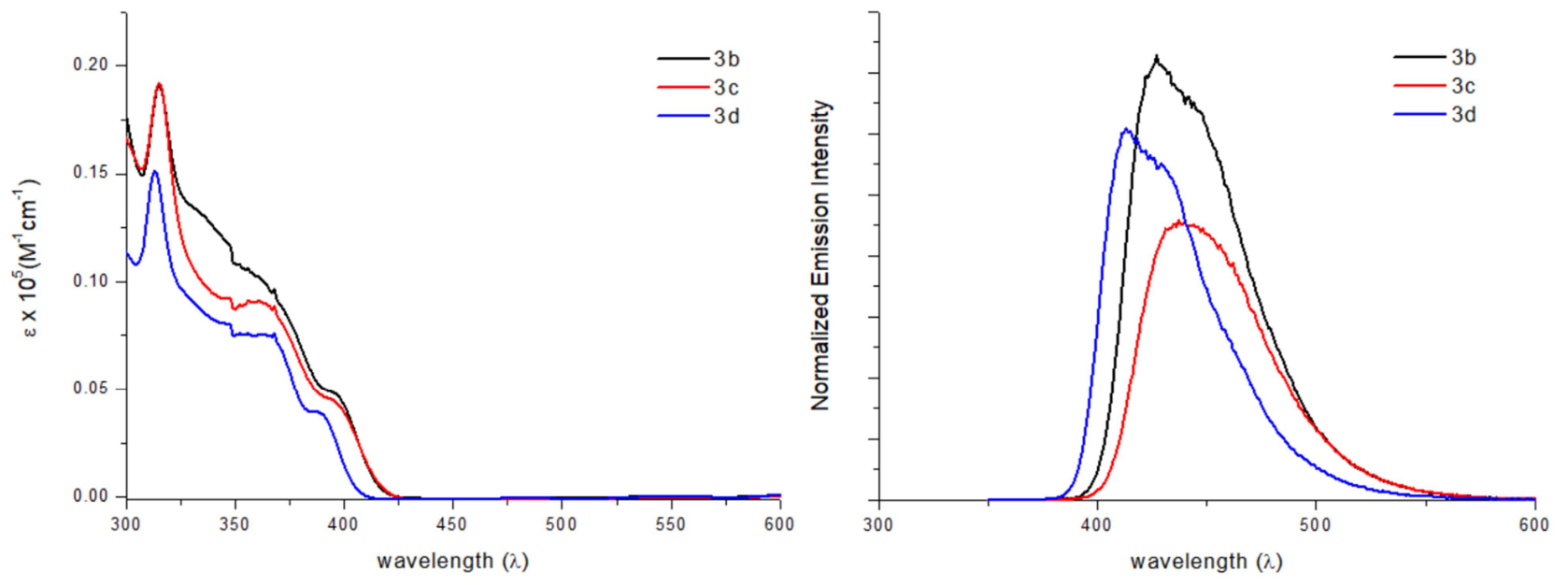

Figure S46. UV-visible absorption (left) and emission (right; excited at $350 \mathrm{~nm}$ ) spectra of 3b, 3c and $\mathbf{3 d}$ in chloroform $\left(2.0 \times 10^{-5} \mathrm{M}\right)$.
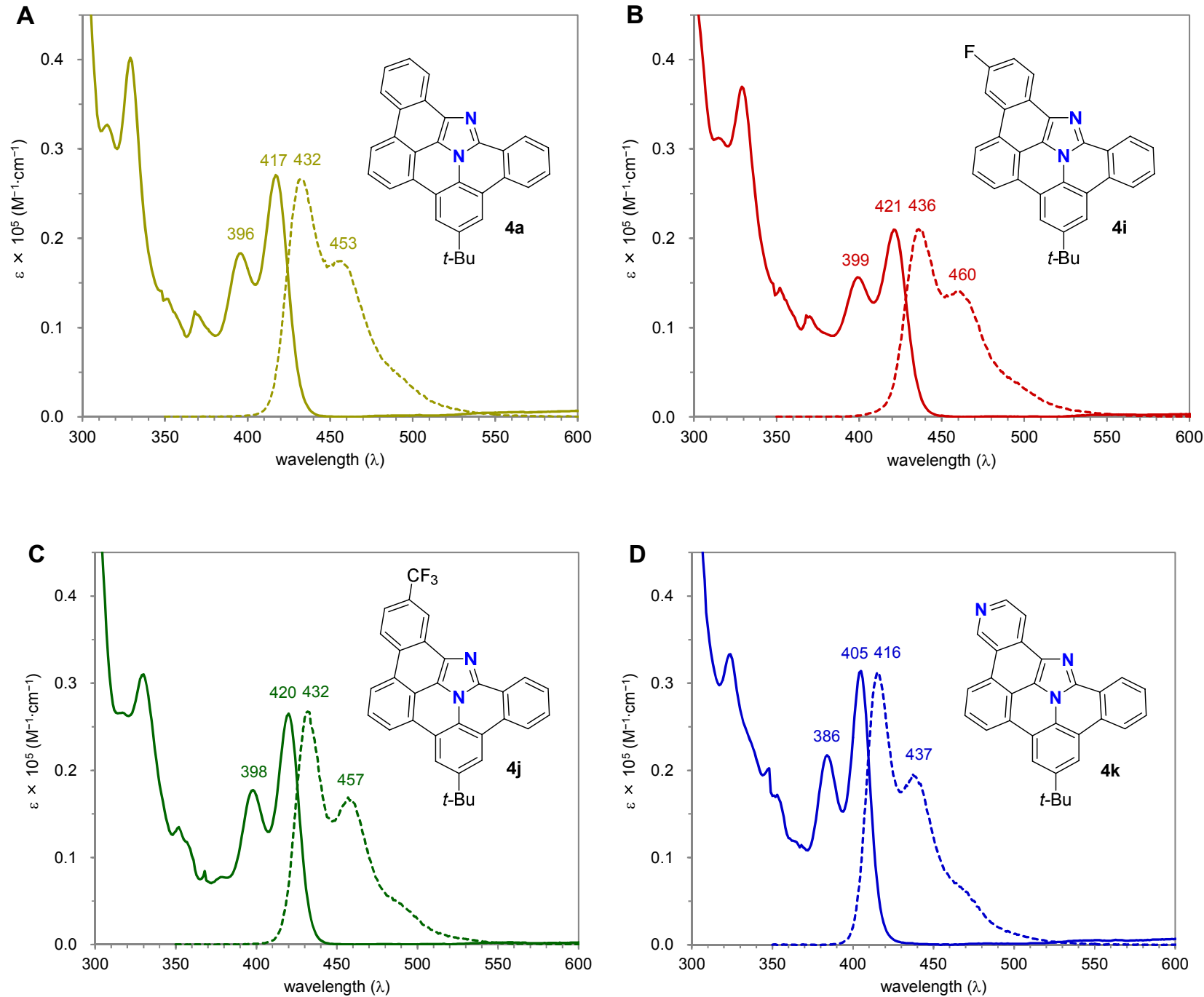

Figure S47. UV-visible absorption (solid line) and emission (dotted line; excited at $350 \mathrm{~nm}$ ) spectra of (A) 4a, (B) 4i, (C) 4j, and (D) $4 \mathbf{k}$. 

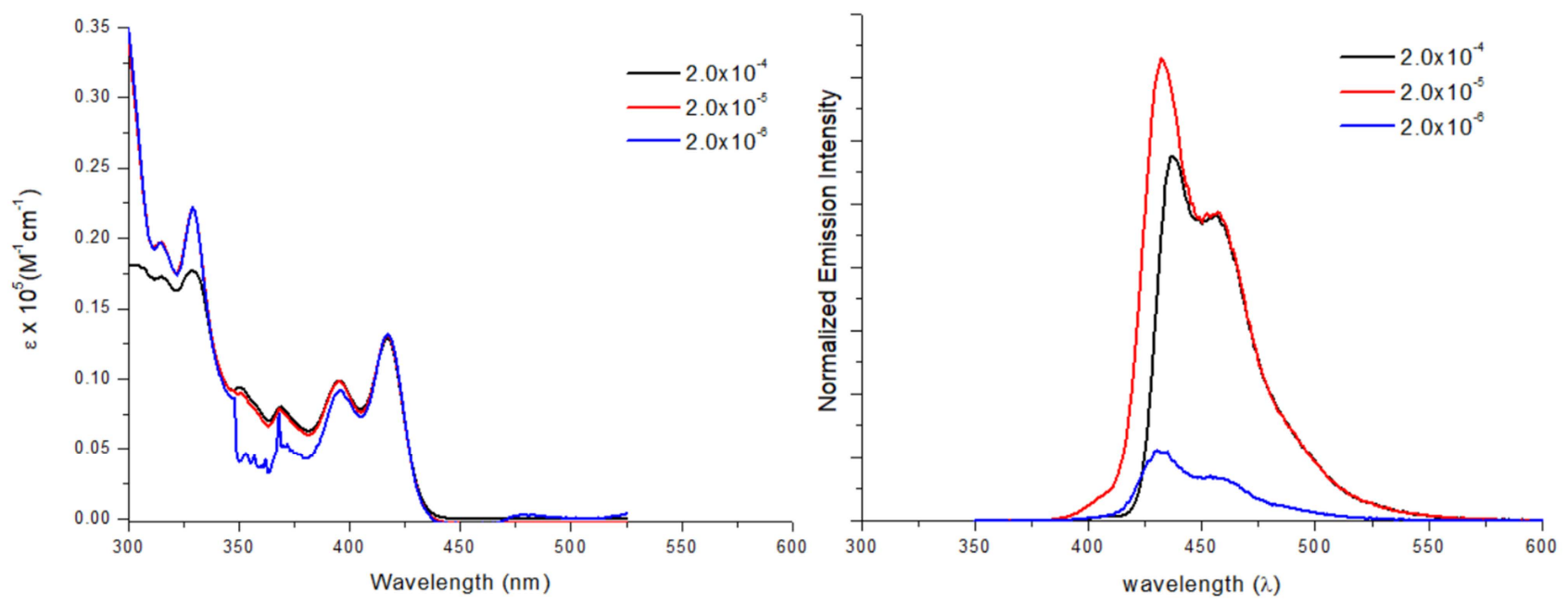

Figure S48. UV-visible absorption and emission (excited at $350 \mathrm{~nm}$ ) spectra of $\mathbf{4 a}$ in chloroform with different concentrations (black: $2.0 \times 10^{-4} \mathrm{M}$, red: $2.0 \times 10^{-5} \mathrm{M}$, blue: $2.0 \times 10^{-6} \mathrm{M}$ ).
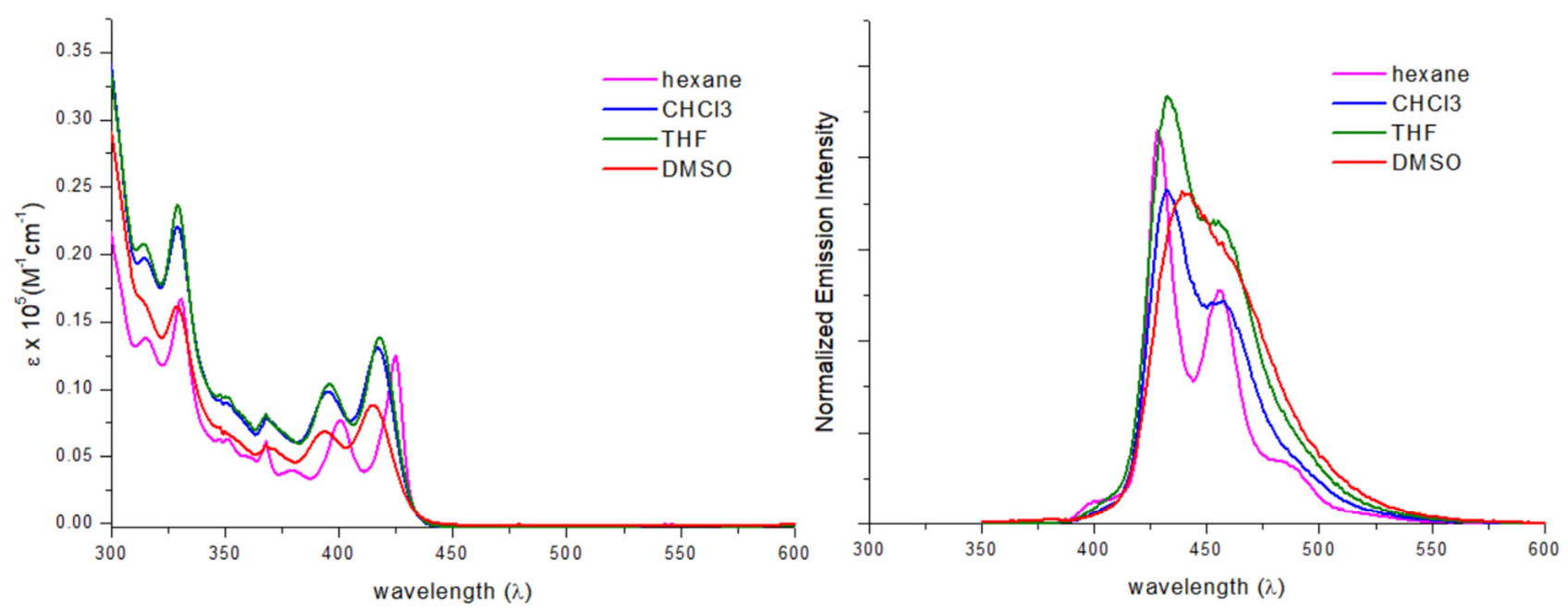

Figure S49. UV-visible absorption and emission (excited at $350 \mathrm{~nm}$ ) spectra of $4 \mathbf{a}$ in different solvents $\left(\mathrm{CHCl}_{3}\right.$, hexane, DMSO, THF, $\left.2.0 \times 10^{-5} \mathrm{M}\right)$. 


\section{Electrochemical Properties}
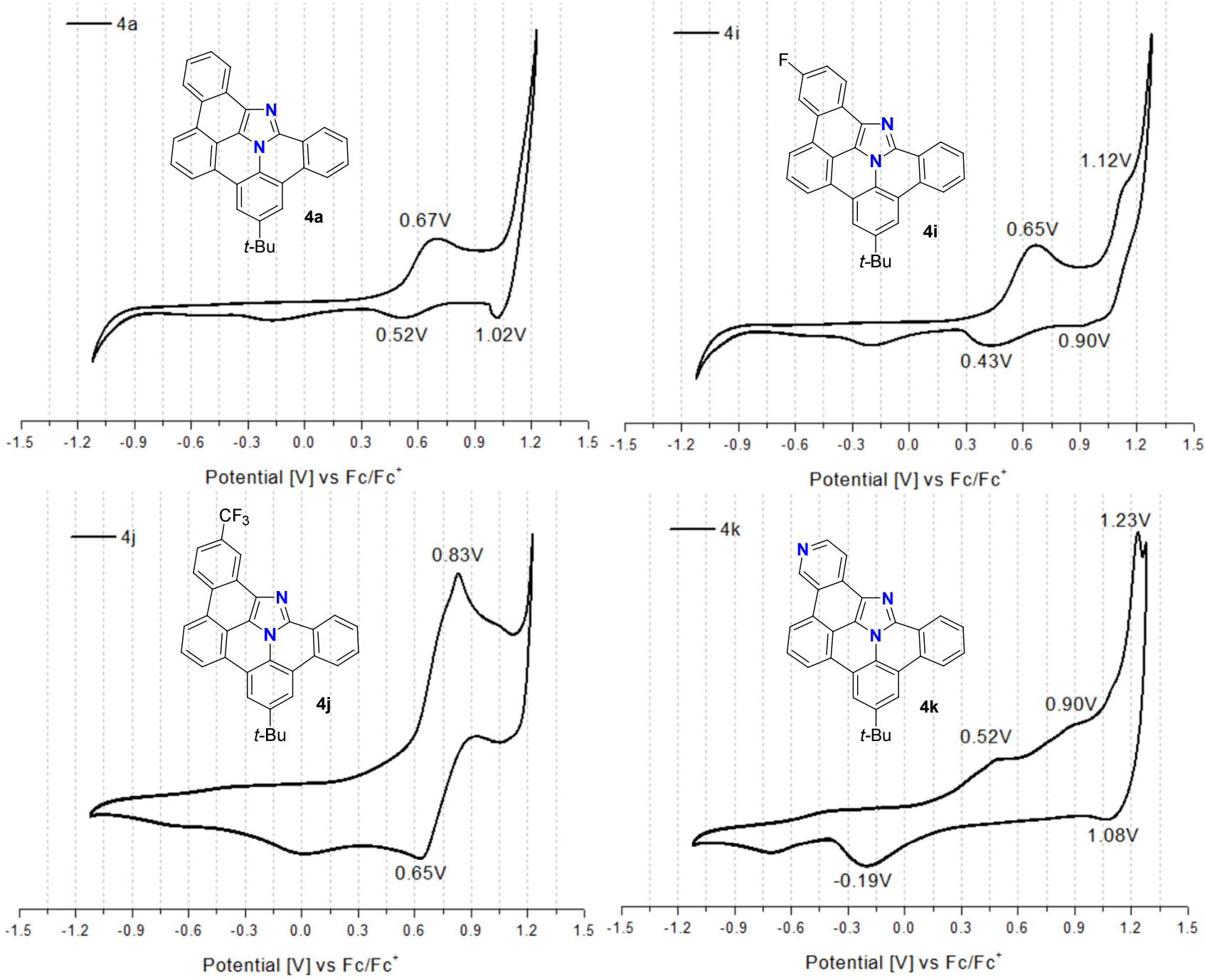

Figure S50. Cyclic voltammogram of $\mathbf{4 a}, \mathbf{4 i}, \mathbf{4 j}$, and $\mathbf{4 k}$ measured with $\mathrm{Bu}_{4} \mathrm{NPF}_{6}$ in $\mathrm{CH}_{2} \mathrm{Cl}_{2}(0.10 \mathrm{M})$ ( $\mathrm{scan}$ rate $=50 \mathrm{mV} / \mathrm{s}$ for $\mathbf{4 a}$ and $\mathbf{4 i} ; 100 \mathrm{mV} / \mathrm{s}$ for $\mathbf{4 j}$ and $\mathbf{4 k}$ ) using $\mathrm{Ag} / \mathrm{Ag}^{+}$as the reference electrode, $\mathrm{Pt}$ as the working electrode, and $\mathrm{Pt}$ wire as the counter electrode. The potential was calibrated against $\mathrm{Fc} / \mathrm{Fc}^{+}$. 


\section{Theoretical Calculations}

The computations were performed using workstation at High-Performance Center for Computational Science, Nanyang Technological University, Singapore. All the calculations were performed by using Gaussian 09 (revision E.1) program ${ }^{4}$ by the B3LYP method ${ }^{5}$ with the $6-31 \mathrm{G}+(\mathrm{d}$ ) basis $\operatorname{set}^{6}$ for structure optimization, vibrational frequency, and TD calculations. All structures were optimized without any symmetry assumptions. The $t$-butyl substituent was omitted in the calculation; $4 \mathbf{a}^{\prime}$ and $\mathbf{4} \mathbf{k}^{\prime}$ represent $4 \mathbf{a}$ and $\mathbf{4 k}$ wherein the $t$-butyl substituent is replaced by a hydrogen atom.

\section{6-1. Structures of Compounds 5, 4a', $4 k^{\prime}$}

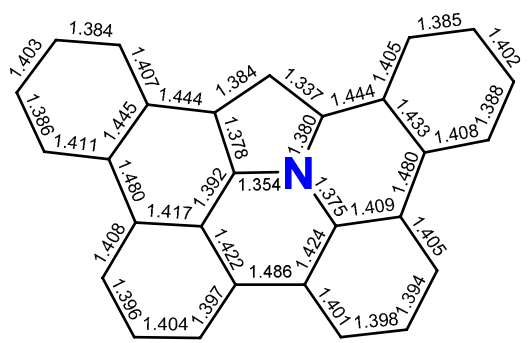

5

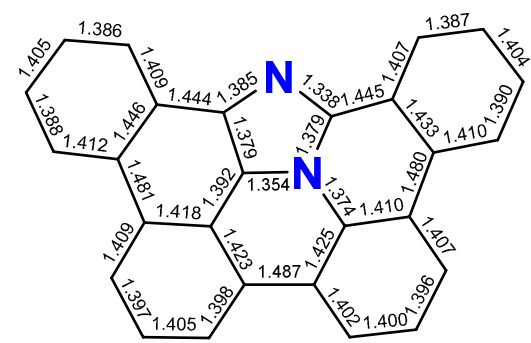

$4 a^{\prime}$

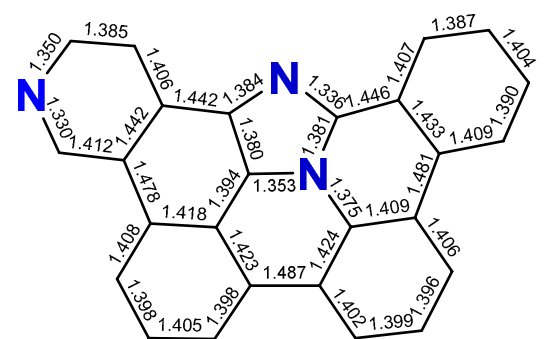

$4 k^{\prime}$

Figure S51. Bond lengths $(\AA ̊)$ of compounds 5, 4a', and 4k' calculated at the B3LYP/6-31+G(d) level of theory.

(4) Frisch, M. J.; Trucks, G. W.; Schlegel, H. B., Scuseria, G. E.; Robb, M. A.; Cheeseman, J. R.; Scalmani, G.; Barone, V.; Mennucci, B.; Petersson, G. A.; Nakatsuji, H.; Caricato, M.; Li, X.; Hratchian, H. P.; Izmaylov, A. F.; Bloino, J.; Zheng, G.; Sonnenberg, J. L.; Hada, M.; Ehara, M.; Toyota, K.; Fukuda, R.; Hasegawa, J.; Ishida, M.; Nakajima, T.; Honda, Y.; Kitao, O.; Nakai, H.; Vreven, T.; Montgomery, Jr., J. A.; Peralta, J. E.; Ogliaro, F.; Bearpark, M.; Heyd, J. J.; Brothers, E.; Kudin, K. N.; Staroverov, V. N.; Keith, T.; Kobayashi, R.; Normand, J.; Raghavachari, K.; Rendell, A.; Burant, J. C.; Iyengar, S. S.; Tomasi, J.; Cossi, M.; Rega, N.; Millam, J. M.; Klene, M.; Knox, J. E.; Cross, J. B.; Bakken, V.; Adamo, C.; Jaramillo, J.; Gomperts, R.; Stratmann, R. E.; Yazyev, O.; Austin, A. J.; Cammi, R.; Pomelli, C.; Ochterski, J. W.; Martin, R. L.; Morokuma, K.; Zakrzewski, V. G.; Voth, G. A.; Salvador, P.; Dannenberg, J. J.; Dapprich, S.; Daniels, A. D.; Farkas, O.; Foresman, J. B.; Ortiz, J. V.; Cioslowski, J.; Fox, D. J. Gaussian 09, Revision C.01, Gaussian, Inc., Wallingford CT, 2010.

(5) (a) Becke, A. D. J. Chem. Phys. 1993, 98, 5648-5652. (b) Lee, C.; Yang, W.; Parr, R. G. Phys. Rev. B 1998, 37, 785-789.

(6) (a) Hehre, W. J.; Ditchfield, R.; Pople, J. A. J. Chem. Phys. 1972, 56, 2257-2261. (b) Ditchfield, R.; Hehre, W. J.; Pople, J. A. J. Chem. Phys. 1971, 54, 724-728. 
6-2. Molecular Orbitals of Compounds 5, 4a', 4k'

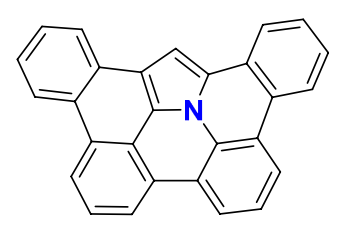

5
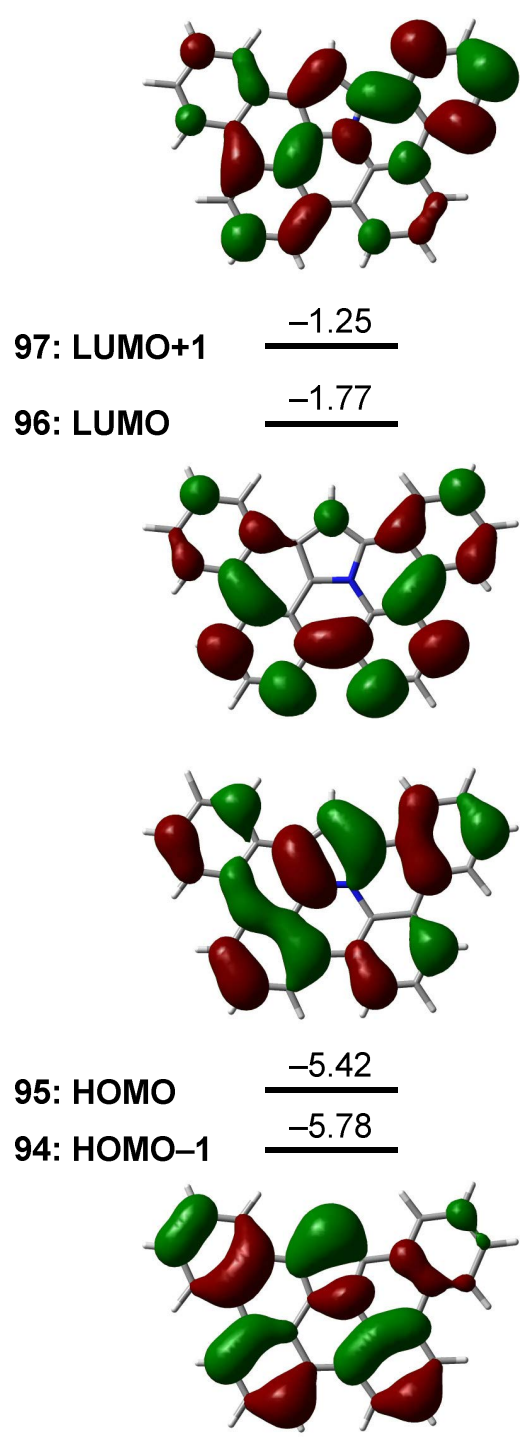
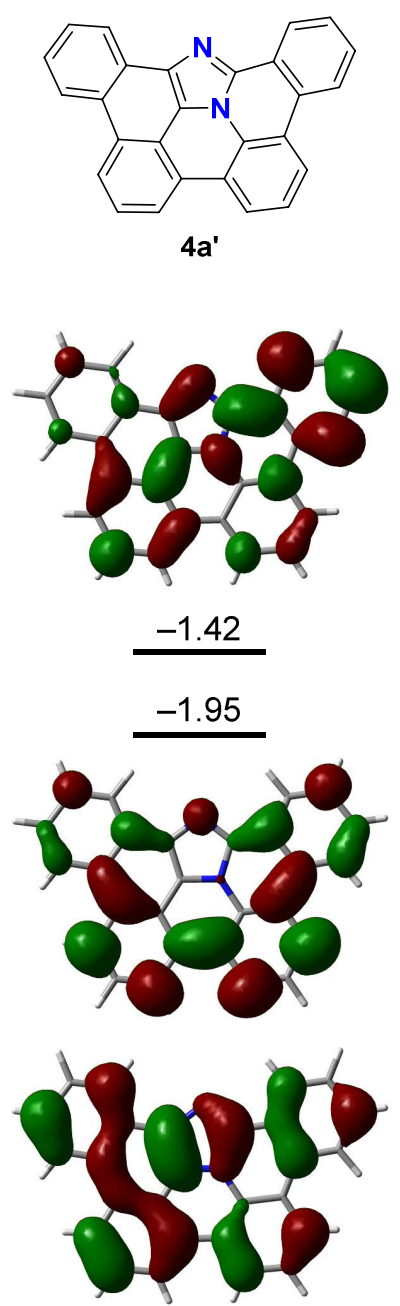

$-5.42$

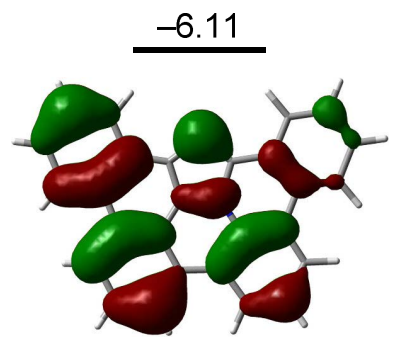

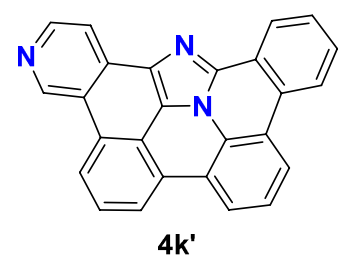
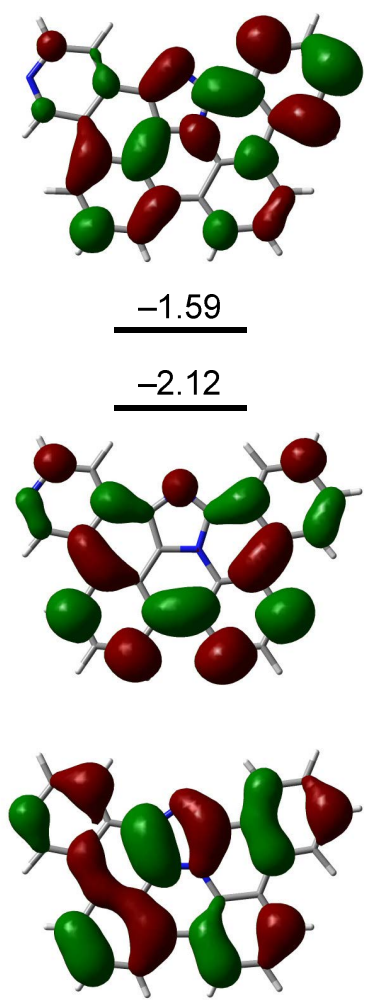

$-5.71$

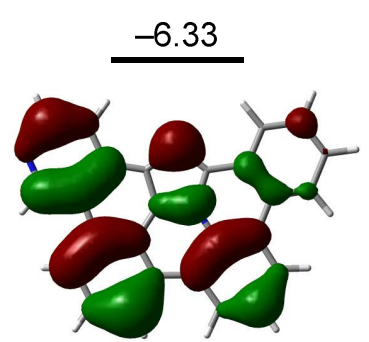

Figure S52. Kohn-Sham molecular orbitals from HOMO-1 to LUMO+1 (eV) of compounds 5, 4a', and $\mathbf{4} \mathbf{k}^{\prime}$ calculated at the B3LYP/6-31+G(d) level of theory. 


\section{6-3. TD-DFT Calculations}

Table S3. Selected wavelengths, oscillator strengths, major electronic transitions of $\mathbf{4 a}$ ' and $\mathbf{4 k}$ ' calculated at the B3LYP/6-31+G(d) level of theory.

\begin{tabular}{|c|c|c|c|c|c|}
\hline \multicolumn{3}{|c|}{$4 a^{\prime}$} & \multicolumn{3}{|c|}{$4 k^{\prime}$} \\
\hline $\begin{array}{l}\text { Wavelength } \\
(\lambda)\end{array}$ & $\begin{array}{l}\text { Oscillator } \\
\text { Strengths }(f)\end{array}$ & Transitions & $\begin{array}{c}\text { Wavelength } \\
(\lambda)\end{array}$ & $\begin{array}{l}\text { Oscillator } \\
\text { Strengths ( } f \text { ) }\end{array}$ & Transitions \\
\hline 413.91 & 0.1644 & $95 \rightarrow 96(0.68901)$ & 397.66 & 0.1780 & $\begin{array}{c}94 \rightarrow 97(-0.10577) \\
95 \rightarrow 96(0.68454)\end{array}$ \\
\hline 361.42 & 0.0468 & $\begin{array}{l}94 \rightarrow 96(-0.36790) \\
95 \rightarrow 97(0.57578)\end{array}$ & 353.34 & 0.0171 & $\begin{array}{l}94 \rightarrow 96(0.41608) \\
95 \rightarrow 97(0.53679)\end{array}$ \\
\hline 352.07 & 0.0107 & $\begin{array}{c}94 \rightarrow 96(-0.10204) \\
95 \rightarrow 98(0.67573) \\
95 \rightarrow 99(-0.11165)\end{array}$ & 342.85 & 0.0285 & $\begin{array}{l}94 \rightarrow 96(-0.11348) \\
95 \rightarrow 97(0.12919) \\
95 \rightarrow 98(0.63868)\end{array}$ \\
\hline 331.36 & 0.2001 & $\begin{array}{c}94 \rightarrow 96(0.48478) \\
95 \rightarrow 97(0.33732) \\
95 \rightarrow 99(-0.32111) \\
95 \rightarrow 100(-0.13929)\end{array}$ & 327.16 & 0.1385 & $\begin{array}{c}93 \rightarrow 96(0.13850) \\
94 \rightarrow 96(-0.35192) \\
95 \rightarrow 97(0.31721) \\
95 \rightarrow 99(0.48001)\end{array}$ \\
\hline 320.69 & 0.0570 & $\begin{array}{c}93 \rightarrow 96(-0.18209) \\
94 \rightarrow 96(0.18007) \\
94 \rightarrow 97(-0.11015) \\
94 \rightarrow 99(0.10564) \\
95 \rightarrow 98(0.13927) \\
95 \rightarrow 99(0.47922) \\
95 \rightarrow 100(-0.35749)\end{array}$ & 315.19 & 0.1287 & $\begin{array}{c}93 \rightarrow 96(-0.18889) \\
94 \rightarrow 96(0.29483) \\
94 \rightarrow 97(-0.13927) \\
94 \rightarrow 99(-0.14323) \\
95 \rightarrow 97(-0.16609) \\
95 \rightarrow 98(0.22255) \\
95 \rightarrow 99(0.38152)\end{array}$ \\
\hline 304.23 & 0.1050 & $\begin{array}{c}94 \rightarrow 96(0.17726) \\
94 \rightarrow 97(-0.41348) \\
95 \rightarrow 97(0.10898) \\
95 \rightarrow 99(0.17258) \\
95 \rightarrow 100(0.45886)\end{array}$ & 303.37 & 0.0892 & $\begin{array}{c}93 \rightarrow 96(0.18874) \\
94 \rightarrow 96(-0.23695) \\
94 \rightarrow 97(-0.23062) \\
94 \rightarrow 100(-0.11078) \\
95 \rightarrow 97(0.12141) \\
95 \rightarrow 99(-0.25560) \\
95 \rightarrow 100(0.45805)\end{array}$ \\
\hline 299.88 & 0.0445 & $\begin{array}{c}93 \rightarrow 96(0.62519) \\
94 \rightarrow 98(-0.14825) \\
95 \rightarrow 99(0.18981)\end{array}$ & 296.51 & 0.0972 & $\begin{array}{c}93 \rightarrow 96(0.59263) \\
94 \rightarrow 97(-0.10426) \\
95 \rightarrow 97(-0.13632) \\
95 \rightarrow 99(-0.11565) \\
95 \rightarrow 100(-0.25680)\end{array}$ \\
\hline 292.38 & 0.1891 & $\begin{array}{c}91 \rightarrow 96(0.10638) \\
93 \rightarrow 96(-0.16360) \\
94 \rightarrow 96(0.12117) \\
94 \rightarrow 97(0.46960) \\
94 \rightarrow 98(-0.29666)\end{array}$ & 290.11 & 0.1824 & $\begin{array}{c}93 \rightarrow 96(-0.10934) \\
94 \rightarrow 96(0.10750) \\
94 \rightarrow 97(0.56948) \\
94 \rightarrow 98(0.25812) \\
95 \rightarrow 99(-0.13637) \\
95 \rightarrow 100(-0.12965)\end{array}$ \\
\hline 285.19 & 0.1709 & $\begin{array}{c}93 \rightarrow 97(-0.30684) \\
94 \rightarrow 96(0.10269) \\
94 \rightarrow 97(0.14557) \\
94 \rightarrow 98(0.50980) \\
94 \rightarrow 100(0.13261) \\
95 \rightarrow 99(0.16993)\end{array}$ & 288.66 & 0.002 & $\begin{array}{c}92 \rightarrow 96(0.65279) \\
92 \rightarrow 97(0.12052) \\
92 \rightarrow 98(-0.12517) \\
92 \rightarrow 100(-0.18805)\end{array}$ \\
\hline 277.03 & 0.0849 & $\begin{array}{c}92 \rightarrow 96(-0.26391) \\
93 \rightarrow 97(0.49678) \\
93 \rightarrow 98(-0.15778) \\
94 \rightarrow 97(0.17717) \\
94 \rightarrow 98(0.23705) \\
95 \rightarrow 100(0.11148)\end{array}$ & 283.80 & 0.1613 & $\begin{array}{c}93 \rightarrow 97(-0.26001) \\
94 \rightarrow 97(-0.16699) \\
94 \rightarrow 98(0.55807) \\
94 \rightarrow 100(-0.14190) \\
95 \rightarrow 99(-0.11862)\end{array}$ \\
\hline
\end{tabular}


4a'

The number of imaginary frequency $=0$

Gibbs Free Energy = -1146.646672 Hartree

$\begin{array}{lrrr}\mathrm{C} & -1.08199800 & -1.38607500 & -0.00000100 \\ \mathrm{C} & -0.67779300 & -0.06797000 & -0.00000300 \\ \mathrm{~N} & 0.67668400 & -0.06635200 & -0.00000300 \\ \mathrm{C} & 1.08253600 & -1.38476700 & -0.00000100 \\ \mathrm{~N} & 0.03026100 & -2.21077100 & -0.00000100 \\ \mathrm{C} & -2.50801000 & -1.61634400 & 0.00000000 \\ \mathrm{C} & -1.43611200 & 1.10019700 & -0.00000100 \\ \mathrm{C} & 1.42617500 & 1.08525600 & -0.00000100 \\ \mathrm{C} & 2.51231600 & -1.59484900 & 0.00000000 \\ \mathrm{C} & -2.84314200 & 0.92269100 & 0.00000000 \\ \mathrm{C} & -3.59834800 & 2.11182400 & 0.00000200 \\ \mathrm{C} & -2.96151300 & 3.35581700 & 0.00000200 \\ \mathrm{C} & -1.56270600 & 3.48969700 & 0.00000100 \\ \mathrm{C} & -0.75440700 & 2.34932300 & -0.00000100 \\ \mathrm{C} & -3.06317200 & -2.91106300 & 0.00000000 \\ \mathrm{C} & -4.43670900 & -3.09755200 & 0.00000100 \\ \mathrm{C} & -5.29219700 & -1.98261700 & 0.00000100 \\ \mathrm{C} & -4.76641000 & -0.69787000 & 0.00000100 \\ \mathrm{C} & -3.37466900 & -0.45910800 & 0.00000000 \\ \mathrm{C} & 0.73268700 & 2.32987100 & -0.00000100 \\ \mathrm{C} & 1.54730000 & 3.47092400 & 0.00000000 \\ \mathrm{C} & 2.94211600 & 3.35646000 & 0.00000100 \\ \mathrm{C} & 3.57919800 & 2.11432000 & 0.00000100 \\ \mathrm{C} & 2.82684100 & 0.92587300 & 0.00000000 \\ \mathrm{C} & 3.37454600 & -0.44943000 & 0.00000000 \\ \mathrm{C} & 4.76412400 & -0.68594100 & 0.00000000 \\ \mathrm{H} & 5.28467800 & -1.97447200 & 0.00000000 \\ \mathrm{C} & 4.42657300 & -3.08570200 & 0.00000100 \\ \mathrm{C} & 3.05290600 & -2.89375500 & 0.00000000 \\ \mathrm{C} & -4.68340100 & 2.08517000 & 0.00000300 \\ \mathrm{H} & -3.57169300 & 4.25535000 & 0.00000400 \\ \mathrm{H} & -1.12888200 & 4.48542700 & 0.00000200 \\ \mathrm{H} & -2.38510200 & -3.75985900 & 0.00000000 \\ \mathrm{H} & -4.85018100 & -4.10282200 & 0.00000200 \\ \mathrm{H} & -6.37009300 & -2.12243100 & 0.00000200 \\ \mathrm{H} & -5.45278700 & 0.14329800 & 0.00000100 \\ \mathrm{H} & 1.09793100 & 4.45919900 & 0.00000100 \\ \mathrm{H} & 3.54601800 & 4.25981200 & 0.00000200 \\ \mathrm{H} & 5.45207700 & 2.08125400 & 0.00000100 \\ \mathrm{H} & 0.15337700 & 0.00000000 \\ \mathrm{H} & & -2.11791700 & 0.00000100 \\ \mathrm{H} & -36194000 & -3.73602100 & 0.00000000\end{array}$

$4 k^{\prime}$

The number of imaginary frequency $=0$

Gibbs Free Energy = -1162.696317 Hartree

$\begin{array}{lrrr}\mathrm{C} & -1.07212900 & -1.40341900 & 0.00000000 \\ \mathrm{C} & -0.67997100 & -0.08030400 & -0.00000100 \\ \mathrm{~N} & 0.67334800 & -0.06903900 & -0.00000100 \\ \mathrm{C} & 1.09000000 & -1.38549000 & -0.00000100 \\ \mathrm{~N} & 0.04597300 & -2.21977400 & -0.00000100 \\ \mathrm{C} & -2.49470700 & -1.63732900 & 0.00000000 \\ \mathrm{C} & -1.44603600 & 1.08444600 & 0.00000000 \\ \mathrm{C} & 1.41567400 & 1.08841500 & 0.00000000 \\ \mathrm{C} & 2.52190900 & -1.58505200 & 0.00000000 \\ \mathrm{C} & -2.85191600 & 0.89969200 & 0.00000000 \\ \mathrm{C} & -3.61759400 & 2.08183400 & 0.00000100 \\ \mathrm{C} & -2.98722300 & 3.32916300 & 0.00000100 \\ \mathrm{C} & -1.58941000 & 3.47194600 & 0.00000100\end{array}$

$\begin{array}{rrr}-0.77249700 & 2.33746300 & 0.00000000 \\ -3.08155400 & -2.91550500 & 0.00000000 \\ -4.46166700 & -3.02877100 & 0.00000000 \\ -4.74940500 & -0.75789200 & 0.00000100 \\ -3.36380300 & -0.48660500 & 0.00000000 \\ 0.71424700 & 2.32802600 & 0.00000000 \\ 1.52091700 & 3.47482100 & 0.00000000 \\ 2.91611600 & 3.36944300 & 0.00000100 \\ 3.56131800 & 2.13133400 & 0.00000000 \\ 2.81707500 & 0.93810000 & 0.00000000 \\ 3.37501700 & -0.43347700 & 0.00000000 \\ 4.76611600 & -0.65961700 & 0.00000000 \\ 5.29565500 & -1.94459900 & 0.00000000 \\ 4.44606200 & -3.06203500 & -0.00000100 \\ 3.07088800 & -2.88000900 & -0.00000100 \\ -4.70221100 & 2.04681900 & 0.00000100 \\ -3.60264600 & 4.22496600 & 0.00000100 \\ -1.16258800 & 4.47058100 & 0.00000100 \\ -2.44664600 & -3.79645600 & 0.00000000 \\ -4.93728400 & -4.00732100 & 0.00000000 \\ -5.45901600 & 0.06659700 & 0.00000100 \\ 1.06496700 & 4.46002100 & 0.00000100 \\ 3.51415100 & 4.27659800 & 0.00000100 \\ 4.64572900 & 2.10553600 & 0.00000100 \\ 5.44822200 & 0.18436300 & 0.00000000 \\ 6.37388200 & -2.08019100 & 0.00000000 \\ 4.86243600 & -4.06562800 & -0.00000100 \\ 2.39175500 & -3.72723100 & -0.00000100 \\ -5.29778300 & -1.96920400 & 0.00000100\end{array}$

5

The number of imaginary frequency $=0$

Gibbs Free Energy = -1130.558037 Hartree

$\begin{array}{lrrr}\mathrm{C} & 1.14114000 & -1.42615700 & -0.00006100 \\ \mathrm{C} & 0.68210900 & -0.11521700 & -0.00003100 \\ \mathrm{~N} & -0.67998100 & -0.10878300 & -0.00003500 \\ \mathrm{C} & -1.14489200 & -1.41746100 & -0.00006500 \\ \mathrm{C} & 2.57653600 & -1.60629700 & -0.00005500 \\ \mathrm{C} & 1.43387600 & 1.06497200 & 0.00001500 \\ \mathrm{C} & -1.42228700 & 1.05266400 & 0.00001400 \\ \mathrm{C} & -2.57979800 & -1.58637600 & -0.00005700 \\ \mathrm{C} & 2.84710400 & 0.92268000 & 0.00002200 \\ \mathrm{C} & 3.58567400 & 2.11883600 & 0.00006700 \\ \mathrm{C} & 2.93936200 & 3.35486600 & 0.00010300 \\ \mathrm{C} & 1.54359700 & 3.46380200 & 0.00009900 \\ \mathrm{C} & 0.75094700 & 2.31270600 & 0.00005700 \\ \mathrm{C} & 3.16915500 & -2.88467000 & -0.00007500 \\ \mathrm{C} & 4.54458000 & -3.04171600 & -0.00006300 \\ \mathrm{C} & 5.37272600 & -1.90927900 & -0.00002800 \\ \mathrm{C} & 4.81388800 & -0.64094600 & -0.00000300 \\ \mathrm{C} & 3.41758000 & -0.43828400 & -0.00001600 \\ \mathrm{C} & -0.72784600 & 2.29477900 & 0.00005500 \\ \mathrm{C} & -1.52618000 & 3.44784600 & 0.00009400 \\ \mathrm{C} & -2.91723000 & 3.35772000 & 0.00009600 \\ \mathrm{C} & -3.56353100 & 2.12248200 & 0.00006000 \\ \mathrm{C} & -2.82904800 & 0.92649500 & 0.00001800 \\ \mathrm{C} & -3.41752400 & -0.42871400 & -0.00001700 \\ \mathrm{C} & -4.81125000 & -0.62673000 & -0.00000200 \\ \mathrm{C} & -5.36869900 & -1.89793100 & -0.00002500 \\ \mathrm{C} & -4.53898900 & -3.02705600 & -0.00006300 \\ \mathrm{C} & -3.16259100 & -2.86771400 & -0.00007600 \\ \mathrm{H} & 4.67044900 & 2.10102400 & 0.00007300 \\ \mathrm{H} & 3.53779400 & 4.26184600 & 0.00013500\end{array}$




$\begin{array}{lrrr}\mathrm{H} & 1.09186800 & 4.45090600 & 0.00013000 \\ \mathrm{H} & 2.52246800 & -3.75820600 & -0.00010000 \\ \mathrm{H} & 4.97930800 & -4.03766900 & -0.00008000 \\ \mathrm{H} & 6.45332100 & -2.02271600 & -0.00001600 \\ \mathrm{H} & 5.47797800 & 0.21736600 & 0.00002800 \\ \mathrm{H} & -1.05898400 & 4.42716000 & 0.00012500 \\ \mathrm{H} & -3.51002600 & 4.26796800 & 0.00012500 \\ \mathrm{H} & -4.64763200 & 2.09900500 & 0.00006300 \\ \mathrm{H} & -5.47506200 & 0.23131800 & 0.00003200 \\ \mathrm{H} & -6.44890100 & -2.01296200 & -0.00001200 \\ \mathrm{H} & -4.97038900 & -4.02415100 & -0.00007900 \\ \mathrm{H} & -2.51430600 & -3.73924400 & -0.00010100 \\ \mathrm{C} & -0.02389500 & -2.25302300 & -0.00005800 \\ \mathrm{H} & -0.05509100 & -3.33382400 & -0.00010800\end{array}$

